\title{
RESEARCH
}

\section{K3 string theory, lattices and moonshine}

CrossMark

\author{
Miranda C. N. Cheng ${ }^{1,2}$, Sarah M. Harrison ${ }^{3}$, Roberto Volpato 4,5,6 and Max Zimet ${ }^{5^{*}}$ (D)
}

*Correspondence:
maxzimet@stanford.edu
${ }^{5}$ Stanford Institute for Theoretical
Physics, Department of Physics,
Stanford University, Stanford, CA
94305, USA
M. C. N. Cheng: On leave from
CNRS, France
Full list of author information is
available at the end of the article

available at the end of the article

\begin{abstract}
In this paper, we address the following two closely related questions. First, we complete the classification of finite symmetry groups of type IIA string theory on $K 3 \times \mathbb{R}^{6}$, where Niemeier lattices play an important role. This extends earlier results by including points in the moduli space with enhanced gauge symmetries in spacetime, or, equivalently, where the world-sheet CFT becomes singular. After classifying the symmetries as abstract groups, we study how they act on the BPS states of the theory. In particular, we classify the conjugacy classes in the T-duality group $\mathrm{O}^{+}\left(\Gamma^{4,20}\right)$ which represent physically distinct symmetries. Subsequently, we make two conjectures regarding the connection between the corresponding twining genera of K3 CFTs and Conway and umbral moonshine, building upon earlier work on the relation between moonshine and the $K 3$ elliptic genus.
\end{abstract}

\section{Contents}

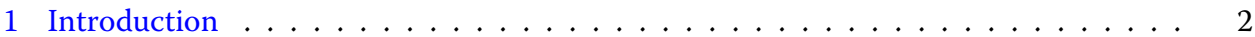

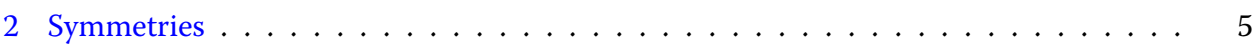

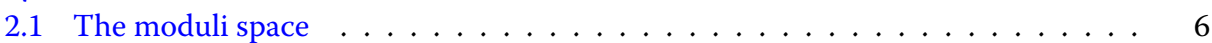

2.2 Symmetry groups . . . . . . . . . . . . . . . . . . . . 7

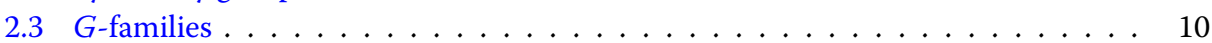

3 Twining genera . . . . . . . . . . . . . . . . . . . . . . 12

3.1 Classification . . . . . . . . . . . . . . . . . . . . 14

3.2 World-sheet parity . . . . . . . . . . . . . . . . . . . 15

3.3 Conway and umbral moonshine $\ldots \ldots \ldots \ldots \ldots$

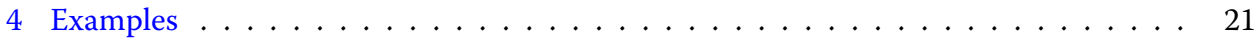

4.1 Geometric symmetries . . . . . . . . . . . . . . . . . . . . . . . . 21

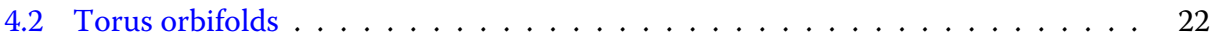

4.3 Landau-Ginzburg orbifolds . . . . . . . . . . . . . . . . . . . . . 24

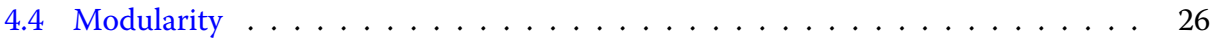

4.5 Evidence for the conjectures $\ldots \ldots \ldots \ldots \ldots \ldots \ldots \ldots$

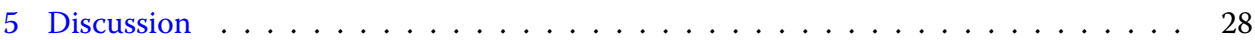

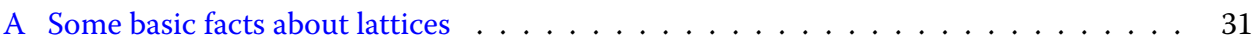

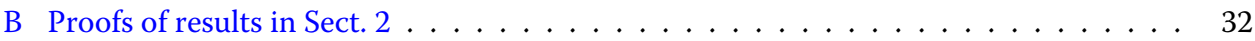

B.1 Proof of Theorem $1 \ldots \ldots \ldots \ldots \ldots$. . . . . . . . . . . . . . 32

B.2 Proof of Theorem $2 \ldots \ldots \ldots \ldots \ldots \ldots$

B.3 Proof of Proposition $3 \ldots \ldots \ldots \ldots$

C Modular groups and multipliers $\ldots \ldots \ldots \ldots$. . . . . . . . . . . 34

D Some classification results . . . . . . . . . . . . . . . . . . . . 35

D.1 Classification of $\mathrm{O}^{+}\left(\Gamma^{4,20}\right)$ conjugacy classes $\ldots \ldots \ldots \ldots \ldots$

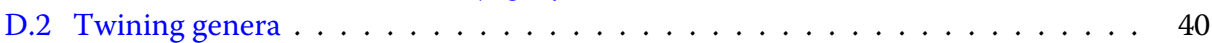

References ......................... 43

(c) The Author(s) 2018. This article is distributed under the terms of the Creative Commons Attribution 4.0 International License (http://creativecommons.org/licenses/by/4.0/), which permits unrestricted use, distribution, and reproduction in any medium, provided you give appropriate credit to the original author(s) and the source, provide a link to the Creative Commons license, and indicate if changes were made. 


\section{Introduction}

In this paper, we study discrete symmetry groups of $K 3$ string theory and their action on the BPS spectrum. $K 3$ surfaces play an important role in various aspects of mathematics and string theory. For instance, type II string compactifications on $K 3 \times T^{d} \times \mathbb{R}^{5-d, 1}$ preserve 16 supersymmetries, leading to various exact results regarding the spectrum of BPS states from both the spacetime and world-sheet points of view. In addition, they provide some of the first instances of both holographic duality and a microscopic description of black hole entropy. Geometrically, the Torelli theorem allows for an exact description of the geometric moduli space and makes it possible to analyze the discrete groups of symplectomorphisms in terms of lattices. In particular, there is an intriguing connection between $K 3$ symmetries and sporadic groups which constitutes the first topic of the current work.

Recall that the sporadic groups are the 26 finite simple groups that do not belong to any of the infinite families of finite simple groups. Their exceptional character raises the following questions: Why do they exist? What geometrical and physical objects do they naturally act on? This is one of the reasons why the discovery of (monstrous) moonshine-relating the representation theory of the largest sporadic simple group and a set of canonical modular functions attached to a chiral 2d CFT-is such a fascinating and important chapter in the study of sporadic groups. On the other hand, the relation of other sporadic groups to the ubiquitous $K 3$ surface is a surprising result that provides another hint about their true raison d'être. In this work, we will relate two properties of sporadic groups: moonshine and $K 3$ symmetries.

The connection between $K 3$ surfaces and sporadic groups first manifested itself in a celebrated theorem by Mukai [73], which was further elucidated by Kondo [69]. Mukai's theorem established a close relation between the Mathieu group $M_{23}$, one of the 26 sporadic groups, and the symmetries of $K 3$ surfaces, in terms of a bijection between (isomorphism classes of) $M_{23}$ subgroups with at least five orbits and (isomorphism classes of) finite groups of $K 3$ symplectomorphisms. A generalization of this classical result to "stringy $K 3$ geometry" was initiated by Gaberdiel, Hohenegger and Volpato in [54], using lattice techniques in a method closely following Kondo's proof of the Mukai theorem. More precisely, the symmetry groups of any nonlinear sigma model (NLSM) on K3, corresponding to any point in the moduli space (2.2) excepting loci corresponding to singular NLSMs, have been classified in [54]. From the spacetime (D-branes) point of view, the results of [54] can be viewed as classifying symplectic autoequivalences (symmetries) of derived categories on $K 3$ surfaces [66]. See also [15] for related discussion on symmetries of appropriately defined moduli spaces relevant for curve counting on $K 3$. The embedding of relevant sublattices of the $K 3$ cohomology lattice into the Leech lattice plays an important role in the analysis, and as a result the classification is phrased in terms of subgroups of the automorphism group $\mathrm{Co}_{0}$ ("Conway zero") of Leech lattice. Recall that there are 24 equivalence classes of 24-dimensional negative-definite even unimodular lattices, called the 24 Niemeier lattices. ${ }^{1}$ All but one of them have root systems of rank 24 ; these are

${ }^{1}$ Note that this is different from the terminology used in [16], where the name "Niemeier lattice" is reserved for the twenty-three 24-dimensional negative-definite even unimodular lattices with non-trivial root systems, and hence excludes the Leech lattice. 
generated by the lattice vectors of length squared two. The only exception is the Leech lattice, which has no root vectors.

The first part of the results of the present paper, consisting in a corollary (Corollary 4) of two mathematical theorems (Theorems 1 and 2), extends this classification to theories corresponding to singular loci in the moduli space of $K 3$ NLSMs. It is necessary to make use of Niemeier lattices other than the Leech lattice (conjecturally all 24 of them, in analogy with [78]) in order to generalize the analysis to include these singular loci. Despite the fact that the type IIA world-sheet theory behaves badly along these loci [5], the full type IIA string theory is not only completely well defined but also possesses special physical relevance in connection with non-Abelian gauge symmetries. Recall that the spacetime gauge group is enhanced from $U(1)^{24}$ to some non-abelian group at these loci, and the ADE-type gauge group is given by the ADE-type singularity of the $K 3$ surface $[5,95]$. The existence of such loci with enhanced gauge symmetries in the moduli space, though not immediately manifest from the world-sheet analysis in type IIA, is clear from the point of view of the dual heterotic $T^{4}$ compactification. In this work, we are interested in finite group symmetries which preserve the $\mathcal{N}=(1,1)$ spacetime supersymmetry from the point of view of type IIA compactifications.

Apart from these physical considerations, another important motivation to understand the discrete symmetries of general type IIA compactifications on $K 3$ surfaces is the following. The $K 3$ surface-sporadic group connection has recently entered the spotlight due to the discovery of new moonshine phenomena, initiated by an observation of Eguchi, Ooguri and Tachikawa (EOT) [46]. The $K 3$ elliptic genus (3.1) is a function which counts BPS states of $K 3$ NLSMs and a loop-space index generalizing the Euler characteristic and the $A$-roof genus, and the observation in [46] suggests that it encodes an infinite-dimensional graded representation of the largest Mathieu sporadic group $M_{24}$. (Note that the group featured in Mukai's theorem, $M_{23}$, is a subgroup of $M_{24}$ as the name suggests.) The existence of a suitable $M_{24}$-module has been eventually proved by Gannon [56], building on previous work in $[19,43,52,53]$. On the other hand, a "natural" construction of this module, in the context, for example, of conformal field theory, string theory or geometry, is still an open problem. A natural guess is that there exists a K3 NLSM with $M_{24}$ acting as its symmetry group. However, the classification result of [54] precludes this solution, and one must find an alternative way to explain Mathieu moonshine. See Sect. 5 for further discussion on this point.

The observation of EOT was truly surprising and led to a surge in activity in the study of (new) moonshine phenomena. Two of the subsequent developments, regarding umbral and Conway moonshines and their relation to K3 NLSMs, motivated the second part of our results which are encapsulated by two conjectures (Conjectures 5 and 6) and further detailed in "Appendix D."

The first development is the discovery of umbral moonshine and its proposed relation to stringy $K 3$ geometry. A succinct and arguably the most natural way to describe Mathieu moonshine is in terms of the relation between a certain set of mock modular forms and $M_{24}$. See, for instance, [28] for an introduction on mock modular forms. Studying Mathieu moonshine from this point of view [21], it was realized in [16,24] that it is but one case of a larger structure, dubbed umbral moonshine. Umbral moonshine consists of a family of 23 moonshine relations corresponding to the 23 Niemeier lattices $N$ with non-trivial root systems: While the automorphism group of a Niemeier lattice dictates the relevant finite 
group $G_{N}$ (cf. (2.12)), the root system of the lattice helps determine a unique (vectorvalued) mock modular form associated with each conjugacy class of $G_{N}$. See Sect. 3.3 for more detail. One of the umbral moonshine conjectures then states that there exists a natural way to associate a graded infinite-dimensional module with the finite group $G_{N}$ such that its graded character coincides with the specified mock modular forms. So far, these modules have been shown to exist $[38,56]$, although, with the exception of a special case [39], a "natural" construction, providing an intuitive interpretation of this vector space and of the action of the corresponding group, is still lacking. Although it is not yet clear whether the structure of vertex operator algebra (VOA; or chiral CFT) is as relevant here as in the classical case of monstrous moonshine, the existence of the generalized umbral moonshine $[13,55]$ suggests that certain key features of VOA should be present in the modules underlying umbral moonshine. Subsequently, motivated by previous work $[78,79]$, the relation between all 23 instances of umbral moonshine and symmetries of $K 3$ NLSMs was suggested in [22] in the form of a proposed relation (3.17) between the umbral moonshine mock modular forms and the $K 3$ elliptic genus twined by certain symmetries (3.5).

The second important development, inspired by the close relation between the Conway group $\mathrm{Co}_{0}$ and stringy $\mathrm{K} 3$ symmetries [54], relates Conway moonshine also to the twined $K 3$ elliptic genus [42]. The Conway moonshine module is a chiral superconformal field theory with $c=12$ and symmetry group $\mathrm{Co}_{0}$, which was first discussed in [49] and further studied in $[40,41]$. Using the Conway module, the authors of [42] associate two (possibly coinciding) Jacobi forms to each conjugacy class of $\mathrm{Co}_{0}$ and conjecture that this set constitutes a complete list of possible $K 3$ twining genera. In particular, it was conjectured that one of the two such Jacobi forms arising from Conway moonshine is attached to each symmetry of any non-singular K3 NLSM. Note that many, but not all, of the functions arising from umbral moonshine [22] and Conway moonshine [42] coincide.

As the first part of our results establishes the importance of several (possibly all) Niemeier lattices in the study of symmetries of $K 3$ string theory, it is natural to suspect that both umbral and Conway moonshine might play a role in describing the action of these symmetry groups on the (BPS) spectrum of $K 3$ string theory. Note that the CFT is not well defined at the singular loci of the module space, and hence we restrict our attention to the non-singular NLSMs when we discuss the (twined) elliptic genus. Motivated by the connection between the stringy $\mathrm{K} 3$ symmetries and moonshine, our analysis of world-sheet parity symmetries of NLSMs (see Sect. 3.2) and results regarding LandauGinzburg orbifolds [17], in this paper we conjecture (Conjecture 5) that the proposed twining genera arising from umbral and Conway moonshine as defined in [22] and [42] capture all of the possible discrete stringy symmetries of any NLSM in the K3 CFT moduli space. Moreover, we conjecture (Conjecture 6) that each of the umbral and Conway moonshine functions satisfying certain basic assumptions (that the symmetry preserves at least four planes in the defining 24-dimensional representation) is realized as the physical twining genus of a certain $K 3$ NLSM. These conjectures pass a few non-trivial tests. In particular, in this paper we also obtain an almost complete classification of conjugacy classes of the discrete T-duality group $O^{+}\left(\Gamma^{4,20}\right)$, as well as a partial classification of the twined $K 3$ elliptic genus using methods independent of moonshine. These classification results, summarized in Table 4, are not only of interest on their own but also provide 
strong evidence for these conjectures which consolidate our understanding of stringy $K 3$ symmetries and the relation between $K 3$ BPS states and moonshine.

Note that the computation of twined elliptic genus determines the twined BPS states counting of black hole states in the $4 \mathrm{~d}$ theory of type IIA string theory compactified on $K 3 \times T^{2}$ via the second quantization formula $[30,35,36,67,84,86]$. The twined BPS states counting functions uniquely determine the representations of the symmetry group underlying the BPS indices. Indeed, the space of BPS states splits into a direct sum of finite-dimensional representations and the twining functions provide the complete list of characters for each such representation. In particular, the group action on the part of the elliptic genus corresponding to the Ramond-Ramond ground states determines its action on the BPS states of D2 branes wrapping cycles on $K 3$ and hence its action on enumerative $K 3$ invariants [15]. Moreover, the twined elliptic genera also determine the BPS spectrum of the corresponding CHL models [20,29-32,67].

The rest of the paper is organized as follows. In Sect. 2, we classify the symmetry groups which arise in type IIA string theory on $K 3 \times \mathbb{R}^{6}$ and preserve the world-sheet $\mathcal{N}=(4,4)$ superconformal algebra in terms of two theorems. This extends the result of [54] to singular points in the moduli space of $K 3$ NLSMs. In Sect. 3, we discuss how these symmetry groups act on the BPS spectrum of the theory. In particular, we present two conjectures relating the twining genera of NLSMs to the functions which feature in umbral and Conway moonshine. In Sect. 4, we summarize all the computations of twining genera in physical models that are known so far, including torus orbifolds and LandauGinzburg orbifolds, and explain how these data provide evidence for our conjectures. Finally, we conclude with a discussion in Sect. 5. A number of appendices include useful information which complements the main text. In "Appendix A," we summarize some basic facts about lattice theory. The proofs of our main theorems discussed in Sect. 2 can be found in "Appendix B." In "Appendix C," we present the arguments that we employ in Sect. 3 to determine the modular properties of certain twining genera. In "Appendix D," we discuss the method we use to classify distinct $O^{+}\left(\Gamma^{4,20}\right)$ conjugacy classes. The result of the classification, as well as the data of the twining genera, is recorded in Table 4.

\section{Symmetries}

In this section, we classify subgroups of $O^{+}\left(\Gamma^{4,20}\right)$ that pointwise fix a positive four-plane, a four-dimensional oriented positive-definite subspace of $\Gamma^{4,20} \otimes_{\mathbb{Z}} \mathbb{R}$. They have the physical interpretation as groups of supersymmetry-preserving discrete symmetries of type IIA string theory on $K 3 \times \mathbb{R}^{6}$. Alternatively, they can be viewed as the symmetry groups of NLSMs on $K 3$ surfaces that commute with the $\mathcal{N}=(4,4)$ superconformal algebra and leave invariant the four R-R ground states corresponding to the spectral flow generators.

We will say such $G \subset O^{+}\left(\Gamma^{4,20}\right)$ is a subgroup of four-plane preserving type and denote the corresponding invariant and co-invariant sublattices by

$$
\Gamma^{G}:=\left\{v \in \Gamma^{4,20} \mid g(v)=v \text { for all } g \in G\right\}, \Gamma_{G}:=\left(\Gamma^{G}\right)^{\perp} \cap \Gamma^{4,20} .
$$

Note that such a group of four-plane preserving type can in general preserve more than just a four-plane, for instance the trivial group.

Our result extends [54] by allowing the co-invariant lattice to contain root vectors. Namely, we include those subgroups of four-plane preserving type such that there exists a 
$v \in \Gamma_{G}$ with $\langle v, v\rangle=-2$, where $\langle\cdot, \cdot\rangle$ denotes the bilinear form of the lattice $\Gamma^{4,20}$. We say that a positive four-plane is a singular positive four-plane if it is orthogonal to some root vector. Physically, they correspond to type IIA compactifications with enhanced gauge symmetry, or to singular NLSMs. The 23 Niemeier lattices with roots play an important role in the analysis of these singular cases.

\subsection{The moduli space}

Let us first review some general properties of NLSMs on K3 (see [3,75]). The moduli space of non-singular NLSMs on $K 3$ with $\mathcal{N}=(4,4)$ supersymmetry is given by an open subset in

$$
\mathcal{M}=(S O(4) \times O(20)) \backslash O^{+}(4,20) / O^{+}\left(\Gamma^{4,20}\right),
$$

where $(S O(4) \times O(20)) \backslash O^{+}(4,20)$ is the Grassmannian of positive four planes $\Pi$ within $\mathbb{R}^{4,20} \cong \Gamma^{4,20} \otimes_{\mathbb{Z}} \mathbb{R}$, and $\Gamma^{4,20}$ is the even unimodular lattice with signature $(4,20)$. The complement in $\mathcal{M}$ of this open subset is the set of singular four planes $\Pi$ (i.e. orthogonal to a root $v \in \Gamma^{4,20}, v^{2}=-2$ ) and corresponds to certain singular limits of NLSMs on K3. The whole space $\mathcal{M}$ is also the moduli space of type IIA string theory at a fixed finite value of $g_{s}$; in particular, points of $\mathcal{M}$ representing singular limits of NLSMs on K3 correspond to perfectly well-defined compactifications of the full non-perturbative type IIA string theory.

The real group $O(4,20):=O(4,20 ; \mathbb{R})$ has four connected components

$$
O(4,20)=O^{++}(4,20) \cup O^{+-}(4,20) \cup O^{-+}(4,20) \cup O^{--}(4,20),
$$

where the elements of

$$
O^{+}(4,20)=O^{++}(4,20) \cup O^{+-}(4,20)
$$

preserve the orientation of positive four planes. ${ }^{2}$ We denote by $O\left(\Gamma^{4,20}\right) \subset O(4,20)$ the group of automorphisms of the lattice $\Gamma^{4,20}$ and define

$$
O^{+}\left(\Gamma^{4,20}\right)=O\left(\Gamma^{4,20}\right) \cap O^{+}(4,20) .
$$

In this work, the lattice $\Gamma^{4,20}$ plays the following roles. Geometrically, $\Gamma^{4,20}$ is the integral cohomology lattice $H^{*}(X, \mathbb{Z})$ with Mukai pairing of a $K 3$ surface $X$, and $\Gamma^{4,20} \otimes_{\mathbb{Z}} \mathbb{R}$ is the real cohomology. Physically, $\Gamma^{4,20}$ is the lattice of D-brane charges, $\Gamma^{4,20} \otimes_{\mathbb{Z}} \mathbb{R}$ is the space of $R-R$ ground states and $\Pi \subset \Gamma^{4,20} \otimes_{\mathbb{Z}} \mathbb{R}$ is the subspace spanned by the four spectral flow generators, i.e., the $\mathrm{R}-\mathrm{R}$ ground states which furnish a $(2,2)$ representation of the $S U(2)_{L} \times S U(2)_{R}$ R-symmetry group. From the point of view of the spacetime physics, the choice of a positive four-plane $\Pi$ is given by a choice of the (spacetime) central charge $Z: \tilde{H}^{1,1}(X, \mathbb{Z}) \rightarrow \mathbb{C}$, where $\tilde{H}^{1,1}(X, \mathbb{Z}):=H^{0,0}(X, \mathbb{Z}) \oplus H^{1,1}(X, \mathbb{Z}) \oplus H^{2,2}(X, \mathbb{Z})$, which determines the mass of supersymmetric D-branes.

Note that in the existing literature, the moduli space is often defined as the quotient of the Grassmannian by the full automorphism group $O\left(\Gamma^{4,20}\right)$ instead of $O^{+}\left(\Gamma^{4,20}\right)$. As

${ }^{2}$ Here and in the following, by "orientation of positive four-planes," we mean each of the two equivalence classes of oriented positive four planes in $\mathbb{R}^{4,20}$ modulo $O(4,20)$ transformations connected to the identity. 
we explain in more detail in Sect. 3.2, dividing by $O^{+}\left(\Gamma^{4,20}\right)$ amounts to distinguishing between NLSMs that are related by world-sheet parity [75]. Due to the existence of symmetries that act differently on the right- and left-moving states of the NLSM, it is crucial for us to identify $O^{+}\left(\Gamma^{4,20}\right)$ instead of $O\left(\Gamma^{4,20}\right)$ as the relevant group of duality.

\subsection{Symmetry groups}

Let us denote by $\mathcal{T}(\Pi)$ the NLSM associated with a given non-singular positive four-plane $\Pi$. With some abuse of notation, we will use the same letter for the lattice automorphism $h \in O^{+}\left(\Gamma^{4,20}\right)$ and the corresponding duality between the two CFTs $\mathcal{T}(\Pi)$ and $\mathcal{T}\left(\Pi^{\prime}\right)$, where $\Pi^{\prime}:=h(\Pi)$. Let $G$ be the group of symmetries of a non-singular NLSM $\mathcal{T}(\Pi)$ preserving the $\mathcal{N}=(4,4)$ superconformal algebra and the four spectral flow generators. It is shown in [54] that $G$ is given by the largest $O\left(\Gamma^{4,20}\right)$-subgroup whose induced action on $\Gamma^{4,20} \otimes_{\mathbb{Z}} \mathbb{R}$ fixes $\Pi$ pointwise, and hence is always a subgroup of $O^{+}\left(\Gamma^{4,20}\right) \subset O\left(\Gamma^{4,20}\right)$. From the spacetime point of view, the group $G$ admits the alternative interpretation as the spacetime-supersymmetry-preserving discrete symmetry group of a six-dimensional type IIA string theory with half-maximal supersymmetry, away from the gauge symmetry enhancement points in the moduli space. More precisely, $G$ is the group of symmetries commuting with all space-time supersymmetries, quotiented by its continuous (gauge) normal subgroup $U(1)^{24}$.

When $\Pi$ is a singular four-plane, the $\operatorname{NLSM} \mathcal{T}(\Pi)$ is not well defined $[4,5,95]$ and it is hence meaningless to talk about the symmetry group of the NLSM in this case. On the other hand, note that the two alternative definitions of the symmetry group $-G$ as the pointwise stabilizer of the subspace $\Pi$ and as the discrete symmetry group of type IIA string theory - can be extended to singular four-plane without any difficulty. One subtlety, however, is that the two definitions are not equivalent for singular models. Indeed, if we define $G_{\text {full }}$ to be the full group of symmetries of the type IIA string model commuting with spacetime supersymmetry, generated by the continuous gauge group $\mathcal{G}$ and by the self-dualities in $\operatorname{Stab}(\Pi)$ and $G_{\text {IIA }}$ to be the quotient $G_{\text {full }} / \mathcal{G}$, then we get

$$
G_{\mathrm{IIA}} \cong \operatorname{Stab}(\Pi) / W
$$

Here, $W \subseteq \operatorname{Stab}(\Pi)$ is the group generated by reflections with respect to hyperplanes $v^{\perp} \subset \Gamma^{4,20} \otimes \mathbb{R}$, for all roots $v \in \Gamma^{4,20} \cap \Pi^{\perp}, v^{2}=-2$, orthogonal to $\Pi$; as we will discuss below, $W$ can be identified with the Weyl group of the continuous group $\mathcal{G}$. We stress that the points in the moduli space where $\operatorname{Stab}(\Pi)$ is non-trivial (which are the most interesting points from our point of view) are exactly the points where the moduli space $\mathcal{M}$ develops orbifold singularities. Among these points, the ones where $W \subseteq \operatorname{Stab}(\Pi)$ is non-trivial can be characterized as the points where the continuous gauge group becomes non-abelian or, equivalently, where the NLSM on K3 becomes singular $[4,5,95]$.

In order to understand why (2.6) holds, let us describe in more detail the definition of $\mathcal{G}$ and $G_{\text {full }}$. The continuous gauge group $\mathcal{G}$ has always rank 24 . It is abelian $\left(\mathcal{G} \cong U(1)^{24}\right)$ if and only if the $\mathrm{K} 3$ sigma model is non-singular. More generally, $\mathcal{G}$ is a semi-simple, simply laced Lie group of rank 24; in the heterotic description of the model, where all gauge bosons correspond to perturbative string states, it is easy to see that the non-abelian part of the Lie algebra of $\mathcal{G}$ is of ADE type, with root lattice isomorphic to the sublattice of 
$\Gamma^{4,20}$ generated by the vectors $v \in \Gamma^{4,20} \cap \Pi^{\perp}$, with $v^{2}=-2[5,95] .{ }^{3}$ In addition to these continuous symmetries, each of the generators of $\operatorname{Stab}(\Pi)$ corresponds to self-dualities of the model and therefore lifts to discrete symmetries of the string theory model. The group $G_{\text {full }}$ is defined as the group generated by $\mathcal{G}$ and by all these self-dualities. Since the self-dualities map gauge bosons into gauge bosons, the continuous group $\mathcal{G}$ is normal in $G_{\text {full }}$, so that the quotient $G_{I I A}=G_{\text {full }} / \mathcal{G}$ is also a group. In order to describe this quotient more explicitly, it is useful to choose a maximal torus $\mathcal{H} \cong U(1)^{24}$ of $\mathcal{G}$. In the type IIA description, the gauge bosons of $\mathcal{H}$ correspond to R-R fundamental string states, while the remaining gauge bosons are given by $\mathrm{D}$-branes getting massless at this particular point in the moduli space [85]. Given such a choice, $\Gamma^{4,20}$ can be interpreted as the lattice of electric charges with respect to $\mathcal{H}$. Since the dualities in $\operatorname{Stab}(\Pi) \subseteq O^{+}\left(\Gamma^{4,20}\right)$ stabilize this lattice, their lifts to $G_{\text {full }}$ can be chosen to be in the normalizer $N \equiv N_{G_{\text {full }}}(\mathcal{H})$ of $\mathcal{H}$ in $G_{\text {full }}$. Notice that the group $G_{\text {full }}$ does not depend on the choice of $\mathcal{H}$ : Since all maximal tori are conjugated within $\mathcal{G}$, a different choice $\mathcal{H}^{\prime}=g \mathcal{H} g^{-1}, g \in \mathcal{G}$, just leads to a different choice of the $G_{\text {full }}$-generators, which get conjugated by $g$. Since $G_{\text {full }}$ can be generated by elements in $\mathcal{G}$ and in the normalizer $N$ of $\mathcal{H}$, we get

$$
G_{\text {full }} / \mathcal{G} \cong N / \mathcal{N}
$$

where $\mathcal{N}:=N \cap \mathcal{G}$ is the normalizer of $\mathcal{H}$ within $\mathcal{G}$. The group $N$ has a well-defined action on the gauge bosons of $\mathcal{H}$ and, consequently, on the lattice of charges $\Gamma^{4,20}$. The condition that $G_{\text {full }}$ commutes with space-time supersymmetry implies that $N$ acts trivially on the four gauge bosons in the gravity supermultiplet (graviphotons), so that the action on $\Gamma^{4,20}$ is via lattice automorphisms in $\operatorname{Stab}(\Pi)$. The homomorphism $N \rightarrow \operatorname{Stab}(\Pi)$ is surjective by construction of $G_{\text {full }}$ and $N$, and its kernel is the maximal torus $\mathcal{H}$, so that we have an isomorphism $N / \mathcal{H} \cong \operatorname{Stab}(\Pi)$. The group $N / \mathcal{H}$ contains, as a normal subgroup, the Weyl group $\mathcal{N} / \mathcal{H}$, which can be identified via this isomorphism with the group $W \subseteq \operatorname{Stab}(\Pi)$ generated by root reflections. Thus, we finally obtain

$$
G_{\text {IIA }}=G_{\text {full }} / \mathcal{G} \cong N / \mathcal{N} \cong(N / \mathcal{H}) /(\mathcal{N} / \mathcal{H}) \cong \operatorname{Stab}(\Pi) / W
$$

While $G_{\text {IIA }}$ is the most interesting group from the point of view of string theory, the group $\operatorname{Stab}(\Pi)$ admits a more direct mathematical definition. Furthermore, by (2.6), it is straightforward to recover $G_{\text {IIA }}$ once $\operatorname{Stab}(\Pi)$ is known. As a result, we will mainly focus on $\operatorname{Stab}(\Pi)$ in this section. In terms of the symplectic autoequivalencies of the bounded derived category $\mathrm{D}^{\mathrm{b}}(\operatorname{Coh}(X))$ of coherent sheaves of a $K 3$ surface $X$, allowing for the orthogonal complement of $\Pi$ in $\Gamma^{4,20}$ to contain roots amounts to relaxing the stability condition in $[8,66]$ to allow for the central charge $Z: \tilde{H}^{1,1}(X, \mathbb{Z}) \rightarrow \mathbb{C}$ to vanish on some $\delta$ with $\delta^{2}=-2$.

Let us now consider the problem of classifying all $\mathrm{O}^{+}\left(\Gamma^{4,20}\right)$ subgroups of four-plane fixing type, including those involving singular four planes. Notice that by definition the invariant lattice $\Gamma^{G}$ has signature $(4, d)$ for some $0 \leq d \leq 20$, and hence the co-invariant lattice $\Gamma_{G}$ is negative definite of rank $20-d$.

${ }^{3}$ While the Lie algebra of $\mathcal{G}$ is uniquely determined by this description, it is an interesting problem to determine the precise group $\mathcal{G}$ acting faithfully on the states of the string theory. We will not try to answer this question here, since it is not important for our subsequent discussion. 
In [54], it is shown that if $\Gamma_{G}$ contains no roots, then it can be primitively embedded into the Leech lattice $\Lambda$ (taken negative definite)

$$
i: \Gamma_{G} \hookrightarrow \Lambda
$$

and that $G$ is isomorphic to a subgroup $\hat{G}$ of the Conway group $C o_{0} \cong O(\Lambda)$. More precisely, $\hat{G} \subset C o_{0}$ acts faithfully on $\Lambda_{\hat{G}}:=i\left(\Gamma_{G}\right) \subset \Lambda$ and fixes pointwise the orthogonal complement $\Lambda^{\hat{G}}=\left(\Lambda_{\hat{G}}\right)^{\perp} \cap \Lambda$.

In order to generalize the classification of the symmetry groups $G$ to singular four planes, we have to consider the case where $\Gamma_{G}$ contains a root. It is clear that in this case, lattices with non-trivial root systems-i.e., Niemeier lattices other than the Leech lattice-are necessary for the embedding. In fact, in this case, the co-invariant lattices can be always embedded into one of the Niemeier lattices, as we show with the following theorem.

Theorem 1 Let $G$ be a subgroup of $O^{+}\left(\Gamma^{4,20}\right)$ fixing pointwise a sublattice $\Gamma^{G}$ of signature $(4, d), d \geq 0$. Then, there exists a primitive embedding $i$ of the orthogonal complement $\Gamma_{G}$ into some negative-definite rank 24 even unimodular lattice (Niemeier lattice) $\mathrm{N}$

$$
i: \Gamma_{G} \hookrightarrow N
$$

Furthermore, the action of $\hat{G}:=i G i^{-1}$ on $i\left(\Gamma_{G}\right)$ extends uniquely to a group of automorphisms of $N$ that fixes pointwise the orthogonal complement of $i\left(\Gamma_{G}\right)$. If $\Gamma_{G}$ has no roots, then $N$ can be chosen to be the Leech lattice.

Proof See "Appendix B.1."

Note that the embedding is generically far from unique, and often $\Gamma_{G}$ can be embedded in more than one Niemeier lattice $N$. At the same time, we believe that all Niemeier lattices are necessary in order to embed all $\Gamma_{G}$ as in (2.10). In particular, in a geometric context it was conjectured in [78] that for each of the 24 Niemeier lattices $N$ there exists a (non-algebraic) $K 3$ surface $X$ whose Picard lattice $P(X)$ can be primitively embedded only in $N$. This conjecture has been proven for all but two Niemeier lattices: those with root systems $A_{24}$ and $2 A_{12}$. It is possible to find an appropriate choice of the B-field such that the orthogonal complement lattice $\Gamma_{G}$ contains the Picard lattice. Therefore, we expect all Niemeier lattices (and not just the Leech lattice) play a role in the study of physical symmetries of type IIA string theory on $K 3$.

By Theorem 1, every group of symmetries $G$ is isomorphic to a subgroup $\hat{G} \subset O(N)$ of the group of automorphisms of some Niemeier lattice $N$, fixing a sublattice of $N$ of rank at least 4 . In fact, the converse is also true by the following theorem.

Theorem 2 Let $N$ be a (negative definite) Niemeier lattice and $\hat{G}$ be a subgroup of $O(N)$ fixing pointwise a sublattice $N^{\hat{G}}$ of rank $4+d, d \geq 0$. Then, there exists a primitive embedding

$$
f: N_{\hat{G}} \hookrightarrow \Gamma^{4,20}
$$

of the co-invariant sublattice $N_{\hat{G}}:=\left(N^{\hat{G}}\right)^{\perp} \cap N$ into the even unimodular lattice $\Gamma^{4,20}$. Furthermore, the action of $G:=f \hat{G} f^{-1}$ on $f\left(N_{\hat{G}}\right)$ extends uniquely to a group of automorphisms of $\Gamma^{4,20}$ that fixes pointwise the orthogonal complement of $f\left(N_{\hat{G}}\right)$. Therefore, there 
exists a positive four-plane $\Pi$ such that $\operatorname{Stab}(\Pi)$ contains $\hat{G}$ as a subgroup. When $N$ is the Leech lattice, $\Pi$ can be chosen so that its orthogonal complement contains no roots.

Proof See "Appendix B.2."

As we will discuss in the next subsection, for many $G$ arising in the way described above, there exist continuous families of $\Pi$ such that the above statement is true, while for those groups with invariant sublattice of rank exactly four, the family consists of isolated points.

It is now useful to make a comparison to the groups in umbral and Conway moonshine (cf. Sect. 3.3). When $N$ is a Niemeier lattice with roots, the automorphism group $O(N)$ contains as a normal subgroup the Weyl group $W_{N}$, generated by reflections with respect to the hyperplanes orthogonal to the roots. The quotients

$$
G_{N}:=O(N) / W_{N}
$$

are the groups whose representation theory dictates the mock modular forms featuring in umbral moonshine [16]. To uniformize the notation, when $N=\Lambda$ is the Leech lattice, we define $W_{N}$ to be the trivial group and subsequently $G_{N}=O(N)=C o_{0}$. We will refer to these $G_{N}$ as the Niemeier groups. Next, we discuss the properties of $G$ in relation to the Niemeier groups.

Proposition 3 For a given sublattice $\Gamma^{G} \subset \Gamma^{4,20}$ of signature $(4, d)$ with $d \geq 0$, let $G:=\left\{g \in O\left(\Gamma^{4,20}\right) \mid g v=v \quad \forall v \in \Gamma^{G}\right\}$. Suppose that the orthogonal complement $\Gamma_{G}$ can be primitively embedded in the Niemeier lattice $N$, so that $G$ is isomorphic to a subgroup $\hat{G} \subset O(N)$. Then:

1. $\hat{G}$ has non-trivial intersection with the Weyl group $W_{N}$ if and only if $\Gamma_{G}$ contains some root.

2. if $\Gamma_{G}$ has no roots, then $G$ is isomorphic to a subgroup of $G_{N}:=O(N) / W(N)$.

Proof See "Appendix B.3."

From the above theorems and proposition, we led to the following corollary for the stringy $K 3$ symmetries:

Corollary 4 Consider type IIA string theory compactified on a K3 surface X corresponding to a point in the moduli space $\mathcal{M}$ (cf. (2.2)).

1. The supersymmetry-preserving discrete symmetry groups $G_{\text {IIA }}$ that are realized somewhere in $\mathcal{M}$ are in bijection with the four-plane preserving subgroups of the Niemeier groups $G_{N}$.

2. Consider the sublattice of the D-brane lattice $H^{*}(X, \mathbb{Z}) \cong \Gamma^{4,20}$ orthogonal to the

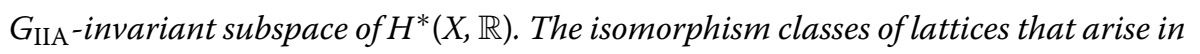
this way somewhere in $\mathcal{M}$ are in bijection with the isomorphism classes of co-invariant lattice $N_{\hat{G}}$, with $N$ a Niemeier lattice and $\hat{G} \subseteq G_{N}$ a four-plane preserving subgroup of the corresponding Niemeier group.

\subsection{G-families}

It is useful to consider families of positive four planes that share certain symmetries, with the equivalence relation given by the action of $O^{+}\left(\Gamma^{4,20}\right)$ taken into account. Let $G$ be a $\mathrm{O}^{+}\left(\Gamma^{4,20}\right)$-subgroup of four-plane fixing type. We define 


$$
\mathcal{F}_{G}:=\left\{\Pi \subseteq \Gamma^{G} \otimes \mathbb{R}, \operatorname{sign}(\Pi)=(4,0)\right\} / \mathcal{N}_{O^{+}\left(\Gamma^{4,20}\right)}(G),
$$

where

$$
\mathcal{N}_{O^{+}\left(\Gamma^{4,20}\right)}(G)=\left\{h \in O^{+}\left(\Gamma^{4,20}\right) \mid h G h^{-1}=G\right\}
$$

is the normalizer of $G$ inside $O^{+}\left(\Gamma^{4,20}\right)$ and corresponds to the subgroup of $O^{+}\left(\Gamma^{4,20}\right)$ that fixes the lattice $\Gamma^{G}$ setwise. Let $d$ be such that the invariant lattice $\Gamma^{G}$ has signature $(4, d)$. Then, $\mathcal{F}_{G}$ is a Grassmannian parametrizing four-dimensional positive-definite subspaces within $\Gamma^{G} \otimes_{\mathbb{Z}} \mathbb{R} \cong \mathbb{R}^{4, d}$, modulo the group $\mathcal{N}_{O^{+}\left(\Gamma^{4,20)}\right.}(G)$ of physical dualities. The family $\mathcal{F}_{G}$ admits a description as a double coset

$$
\mathcal{F}_{G} \cong S O(4) \times O(d) \backslash O^{+}\left(\Gamma^{G} \otimes_{\mathbb{Z}} \mathbb{R}\right) / \mathcal{N}_{O^{+}\left(\Gamma^{4,20}\right)}(G),
$$

which makes manifest that $\mathcal{F}_{G}$ has real dimension $4 d$ and is connected: When $d=0$, the group $O^{+}\left(\Gamma^{G} \otimes_{\mathbb{Z}} \mathbb{R}\right) \cong S O(4)$ has one connected component, while for $d>0$ the two connected components of $O^{+}\left(\Gamma^{G} \otimes_{\mathbb{Z}} \mathbb{R}\right) \cong O^{+}(4, d)$ are identified via $O(d)$. In the following, we will often identify families $\mathcal{F}_{G}$ and $\mathcal{F}_{G^{\prime}}$ that are conjugated in $O^{+}\left(\Gamma^{4,20}\right)$ :

$$
\mathcal{F}_{G} \sim \mathcal{F}_{G^{\prime}} \quad \text { if } \quad G^{\prime}=h G h^{-1} \quad \text { for some } \quad h \in O^{+}\left(\Gamma^{4,20}\right) .
$$

Physically, we are motivated to study $\mathcal{F}_{G}$ for the following reason. From the fact that a positive four-plane defines a $K 3 \mathrm{NLSM}, \mathcal{F}_{G}$ can be physically interpreted as a family of $K 3$ NLSMs with symmetry groups which contain $G$. As we will see, the connectedness of $\mathcal{F}_{G}$ and the continuity argument we present in Sect. 3 then guarantee that all theories $\mathcal{T}(\Pi)$ for $\Pi \in \mathcal{F}_{G}$ have the same twining genera $\mathcal{Z}_{g}(\mathcal{T}(\Pi) ; \tau, z)$ for all $g \in G$.

We close this section with a few useful properties of $\mathcal{F}_{G}$. We would like to know whether a given family $\mathcal{F}_{G}$ contains any singular positive four-plane. First, let us distinguish between the following two cases:

(1) $\Gamma_{G}$ contains no roots

(2) $\Gamma_{G}$ contains roots

By definition, case (1) contains some non-singular four planes, while case (2) contains only singular models. It is natural to ask under what circumstances does a family in case (1) contain singular four planes. In what follows, we collect the answer for a few interesting cases:

- if $G$ is a group of geometric symmetries (i.e., if $G$ arises as a group of hyper-Kähler preserving symmetries of a $K 3$ surface), then the corresponding family $\mathcal{F}_{G}$ contains some singular models. To see this, first recall that a necessary and sufficient condition for $G$ to be geometric is that the invariant lattice $\Gamma^{G}$ contains an even unimodular $\Gamma^{1,1} \subset \Gamma^{G}$. In this case, one can take any root $v \in \Gamma^{1,1}$ and notice that $v^{\perp} \cap\left(\Gamma^{G} \otimes_{\mathbb{Z}} \mathbb{R}\right)$ has signature $(4, d-1)$, so it contains some $\Pi$ of signature $(4,0)$ that is by definition singular.

- If $\Gamma^{G}$ has rank exactly four, then $\mathcal{F}_{G}$ consists of a single point, and if we are in case 1 above, this is by definition non-singular. 
- If the defining 24-dimensional representation of $G$ is not a permutation representation, then all four planes in $\mathcal{F}_{G}$ are non-singular. This can be seen as follows. For each $\Pi$ in the family $\mathcal{F}_{G}$, one can show, using techniques analogous to the proof of Theorem 2, that the orthogonal sublattice $\Gamma_{\Pi}:=\Pi^{\perp} \cap \Gamma^{4,20}$ can be primitively embedded in some Niemeier lattice $N$ (possibly depending on $\Pi$ ). This implies that also $\Gamma_{G} \subset \Gamma_{\Pi}$ can be primitively embedded in $N$. Recall that the defining 24-dimensional representation is a permutation representation for all subgroups of the Niemeier group $G_{N}$ unless $N$ is the Leech lattice; indeed, when $N$ is not the Leech lattice, $G_{N}$ is just the group of automorphisms of the Dynkin diagram of the corresponding root lattice ([26], chapter 16) and therefore acts by permutations on a basis of 24 simple roots. By hypothesis, $\Gamma_{G}$ has no roots, so that by Proposition $3 \mathrm{G}$ must be isomorphic to a subgroup of $G_{N}$. The only $N$ such that the 24-dimensional representation of $G_{N}$ is not a permutation representation is the Leech lattice. We conclude that, for all $\Pi$ in $\mathcal{F}_{G}, \Gamma_{\Pi}$ can be embedded in the Leech lattice, and therefore it cannot contain any root.

On the other hand, assuming a family in case (1) does contain a singular four-plane, we can deduce the following result about $\Gamma_{G}$ :

- If $\mathcal{F}_{G}$ contains some singular four-plane $\Pi$, then $\Gamma_{G}$ can be embedded in some Niemeier lattice $N$ with roots, so that $G$ is isomorphic to a subgroup of the Niemeier group $O(N) / W_{N}$. The argument for this is analogous to the previous statement. The sublattice $\Gamma_{\Pi}:=\Pi^{\perp} \cap \Gamma^{4,20}$ orthogonal to a singular four-plane $\Pi$ can be primitively embedded in some Niemeier lattice $N$. By definition, $\Gamma_{\Pi}$ contains some root and hence $N$ cannot be the Leech lattice. Furthermore, $\Gamma_{G}$ is a primitive sublattice of $\Gamma_{\Pi}$, so it can also be primitively embedded in $N .{ }^{4}$

\section{Twining genera}

In this section, we investigate how the symmetry groups discussed in the previous section act on the BPS spectrum of the theory. In particular, in Sect. 3.3 we will present two conjectures relating the twining genera of NLSMs and the functions featured in umbral and Conway moonshine. In this section, we restrict our attention to non-singular NLSMs as the elliptic genus is otherwise not well defined.

For any non-singular NLSM $\mathcal{T}$ on $K 3$, the elliptic genus $[45,92]$ may be defined as

$$
\mathcal{Z}(\mathcal{T} ; \tau, z)=\operatorname{Tr}_{\mathcal{H}_{R R}}\left(q^{L_{0}-\frac{c}{24}} \bar{q}^{\left.\bar{L}_{0}-\frac{\bar{c}}{24} y^{J_{0}}(-1)^{J_{0}+\bar{J}_{0}}\right)}\right.
$$

where $q:=e^{2 \pi i \tau}, y:=e^{2 \pi i z}$. In the above definition, $\mathcal{H}_{R R}$ denotes the Ramond-Ramond Hilbert space of $\mathcal{T}$, and $L_{0}, \bar{L}_{0}$ and $J_{0}, \bar{J}_{0}$ denote the zero modes of the left- and right-moving Virasoro resp. Cartan generators in the $s u(2)$ level 1 Kac-Moody algebras contained in the $\mathcal{N}=(4,4)$ superconformal algebra with central charges $c=\bar{c}=6$. As is well known, the elliptic genus of a compact theory only receives nonvanishing contributions from the right-moving ground states which have vanishing eigenvalue of $\bar{L}_{0}-\frac{\bar{c}}{24}$, and hence it is holomorphic both in $\tau$ and in $z$. Moreover, $\mathcal{Z}(\mathcal{T} ; \tau, z)$ is a weak Jacobi form of weight zero

${ }^{4}$ However, the converse is not true: It can happen that $\Gamma_{G}$ admits a primitive embedding into a Niemeier lattice while $\mathcal{F}_{G}$ contains no singular model, as exemplified by certain examples when $\Gamma^{G}$ is exactly four-dimensional and $\mathcal{F}_{G}$ contains only an isolated point. 
and index 1, i.e. it satisfies certain growth conditions [47] and is a holomorphic function $\mathbb{H} \times \mathbb{C} \rightarrow \mathbb{C}$ satisfying the following modularity

$$
\phi_{k, m}(\tau, z)=(c \tau+d)^{-k} e^{-2 \pi i m \frac{c z^{2}}{c \tau+d}} \phi_{k, m}\left(\frac{a \tau+b}{c \tau+d}, \frac{z}{c \tau+d}\right) \forall\left(\begin{array}{ll}
a & b \\
c & d
\end{array}\right) \in S L_{2}(\mathbb{Z}),
$$

and quasi-periodicity properties

$$
\phi_{k, m}(\tau, z)=e^{2 \pi i m\left(\ell^{2} \tau+2 \ell z\right)} \phi_{k, m}\left(\tau, z+\ell \tau+\ell^{\prime}\right) \quad \forall\left(\ell, \ell^{\prime}\right) \in \mathbb{Z}^{2},
$$

for $k=0$ and $m=1$. The elliptic genus is a (refined) supersymmetric index and, in particular, is invariant under supersymmetric marginal deformations of the nonlinear sigma model. ${ }^{5}$ Since the moduli space of K3 NLSMs is connected, this means that $\mathcal{Z}(\mathcal{T} ; \tau, z)$ is independent of the particular $K 3$ NLSM $\mathcal{T}$ from which it is calculated. As a result, often we will simply denote it as $\mathcal{Z}(K 3 ; \tau, z)$. Explicitly, it can be expressed in terms of Jacobi theta functions as

$$
\mathcal{Z}(K 3 ; \tau, z)=8 \sum_{i=2}^{4} \frac{\theta_{i}(\tau, z)^{2}}{\theta_{i}(\tau, 0)^{2}}=2 y+20+2 y^{-1}+O(q) .
$$

Let us consider a non-singular NLSM $\mathcal{T}$ with a symmetry group $G$. Then, for each $g \in G$, one can define the twining genus

$$
\mathcal{Z}_{g}(\mathcal{T} ; \tau, z)=\operatorname{Tr}_{\mathcal{H}_{R R}}\left(g q^{L_{0}-\frac{c}{24}} \bar{q}^{\bar{L}_{0}-\frac{\bar{c}}{24}} J^{J_{0}}(-1)^{F_{L}+F_{R}}\right) .
$$

From the usual path integral picture, ${ }^{6}$ one concludes that $\mathcal{Z}_{g}$ is a weak Jacobi form of weight 0 and index 1 for some congruence subgroup $\mathfrak{G}_{g}$ of $S L_{2}(\mathbb{Z})$, possibly with a nontrivial multiplier system (see "Appendix C" for details).

By the same arguments as for the elliptic genus and under standard assumptions about deformations of $\mathcal{N}=(4,4)$ superconformal field theories, the twining genus $\mathcal{Z}_{g}$ is invariant under exactly marginal deformations that preserve supersymmetry and the symmetry generated by $g$. More precisely, consider a group of symmetries $G$ such that the subspace

$$
\mathcal{F}_{G}^{\text {ns }}:=\left\{\Pi \subseteq \mathcal{F}_{G} \mid \Pi \text { is not singular }\right\}
$$

of non-singular positive four planes is non-empty (cf.(2.13)). Note that there is no loss of generality by restricting to non-singular models, since only for these the world-sheet definition of (twined) elliptic genus that we employ in this section applies. Then, we argue that the following is true:

Let $g \in O^{+}\left(\Gamma^{4,20}\right)$ be a group element fixing pointwise a sublattice $\Gamma^{g} \subseteq \Gamma^{4,20}$ of signature $(4, d)$ and such that the co-invariant lattice $\Gamma_{g}$ contains no roots. Then, the family $\mathcal{F}_{g}^{\text {ns }}:=$ $\mathcal{F}_{\langle g\rangle}^{\text {ns }}$ of non-singular four planes with symmetryg is non-empty and connected. Furthermore, if we assume that the operators $L_{0}, \bar{L}_{0}, J_{0}, \bar{J}_{0}$ and $g$ vary continuously under deformations within the family of NLSM corresponding to $\mathcal{F}_{g}^{\text {ns }}$, then the twining genus $\mathcal{Z}_{g}$ is constant on $\mathcal{F}_{g}^{n s}$.

\footnotetext{
${ }^{5}$ This is true except at points in moduli space where a non-compact direction opens up and the CFT is singular. ${ }^{6}$ In the present paper, we make the standard assumption in theoretical physics that the path integral formulation of NLSM yields correct answers.
} 
The proof is an obvious generalization of the arguments showing that the elliptic genus is independent of the moduli. One first defines the twining genus $\mathcal{Z}_{g}$ along any connected path within the family $\mathcal{F}_{g}^{n s}$ and then uses continuity of $L_{0}, \bar{L}_{0}, J_{0}, \bar{J}_{0}$ as well as the discreteness of their spectrum within the relevant space of states to show that $\mathcal{Z}_{g}$ must be actually constant along this path. An even simpler proof can be given if one adopts the equivalent definition of the twining genus $\mathcal{Z}_{g}$ as an equivariant index in the $Q$-cohomology of a halftwisted topological model. In this case, it is sufficient to use the fact that a $g$-invariant and $Q$-exact deformation cannot change the index.

We note that

$$
\mathcal{Z}_{g}(\tau, z)=\mathcal{Z}_{g^{-1}}(\tau,-z)=\mathcal{Z}_{g^{-1}}(\tau, z)
$$

Here, the first equality corresponds to the transformation $\left(\begin{array}{cc}-1 & 0 \\ 0 & -1\end{array}\right) \in S L_{2}(\mathbb{Z})$ and follows from standard path integral arguments. The second equality holds because the space of states carries a representation of the $s u(2)$ algebra contained in the left-moving $\mathcal{N}=4$ algebra, and $s u(2)$ characters are always even.

Finally, a twining genus $\mathcal{Z}_{g}$ is invariant under conjugation by any duality $h \in O^{+}\left(\Gamma^{4,20}\right)$. More precisely, suppose $h$ is a duality between the models $\mathcal{T}$ and $\mathcal{T}^{\prime}$, i.e., an isomorphism between the fields and the states of the two theories that maps the superconformal generators into each other and is compatible with the OPE. Then, the twining genus $\mathcal{Z}_{g}$ defined in the model $\mathcal{T}$ equals the twining genus $\mathcal{Z}_{h g h^{-1}}$ defined in the model $\mathcal{T}^{\prime}$. This follows after noticing that $h$ maps $\left(L_{0}, J_{0}\right)$-eigenspaces in $\mathcal{T}$ into the corresponding eigenspaces in $\mathcal{T}^{\prime}$ and using the cyclic properties of the trace. The effect of a conjugation under a duality in $O\left(\Gamma^{4,20}\right) \backslash O^{+}\left(\Gamma^{4,20}\right)$ is much more subtle and will be discussed in Sect. 3.2.

Using the above results, one can assign a twining genus $\mathcal{Z}_{g}$ to any conjugacy class $[g]$ of $O^{+}\left(\Gamma^{4,20}\right)$ such that $\langle g\rangle$ is a subgroup of four-plane fixing type and that the co-invariant sublattice $\Gamma_{g}$ contains no roots. In principle, $\mathcal{Z}_{g}$ and $\mathcal{Z}_{g^{\prime}}$ are distinct if $g^{\prime}$ is conjugate to neither $g$ nor $g^{-1}$ as elements of $O^{+}\left(\Gamma^{4,20}\right)$, unless accidental coincidences occur. ${ }^{7}$ In the next subsection we will classify the conjugacy classes of $O^{+}\left(\Gamma^{4,20}\right)$.

\subsection{Classification}

While many examples of twining genera have been computed in specific sigma models, a full classification of the corresponding conjugacy classes in $O^{+}\left(\Gamma^{4,20}\right)$ and a complete list of all corresponding twining genera are still an open problem. In this work, we solve the first problem for all but one of the 42 possibilities (labeled by conjugacy classes of $\mathrm{Co}_{0}$ ).

As a first step in this classification problem, it is useful to consider the eigenvalues of $\mathrm{O}^{+}\left(\Gamma^{4,20}\right)$-elements in the defining 24-dimensional representation, denoted below simply by $\rho_{24}: O^{+}\left(\Gamma^{4,20}\right) \rightarrow \operatorname{End}\left(V_{24}\right)$, given by $V_{24} \cong \Gamma^{4,20} \otimes_{\mathbb{Z}} \mathbb{R}$. (This is also the representation on the $24 \mathrm{R}-\mathrm{R}$ ground states in a sigma model $\mathcal{T}(\Pi) \in \mathcal{F}_{g}$.) It is convenient to encode such information in the form of a Frame shape, i.e. a symbol

$$
\pi_{g}:=\prod_{\ell \mid N} \ell^{k_{\ell}}
$$

${ }^{7}$ Coincidences like this occur, for example, when the dimension of the relevant space of modular forms is small. See Sects. 4.4 and 4.5 for more details. 
where $N=o(g)$ is the order of $g$. The integers $k_{\ell} \in \mathbb{Z}$ are defined by

$$
\operatorname{det}\left(t \mathbf{1}_{24}-\rho_{24}(g)\right)=\prod_{\ell \mid N}\left(t^{\ell}-1\right)^{k_{\ell}} .
$$

When $g$ acts as a permutation of the vectors in some basis of $\Gamma^{4,20} \otimes_{\mathbb{Z}} \mathbb{R}$, all $k_{\ell}$ are nonnegative and the Frame shape coincides with the cycle shape of the permutation. We will say that a Frame shape is a four-plane preserving Frame shape if it coincides with the Frame shape of an element of a four-plane preserving subgroup of $O\left(\Gamma^{4,20}\right)$, as defined in Sect. 2. Explicitly, a Frame shape is a four-plane preserving Frame shape if and only if $\sum_{\ell} k_{\ell} \geq 4$, corresponding to the fact that the eigenvalue 1 must be repeated at least four times. A salient feature shared by the Frame shapes of all Niemeier groups that correspond to Niemeier lattices with non-trivial root systems (and hence not given by the Leech lattice) is that they are all cycle shapes, and this is not true for some of the Conway Frame shapes.

One can explicitly check, by using Theorem 1, that such four-plane preserving Frame shapes of $\mathrm{O}^{+}\left(\Gamma^{4,20}\right)$ are precisely the 42 four-plane preserving Frame shapes of $\mathrm{Co}_{0}$, corresponding to the 42 four-plane preserving conjugacy classes of $\mathrm{Co}_{0}$. Moreover, if $g, g^{\prime} \in O^{+}\left(\Gamma^{4,20}\right)$ have the same Frame shape, then the co-invariant sublattices $\Gamma_{g}$ and $\Gamma_{g^{\prime}}$ are isomorphic

$$
\pi_{g}=\pi_{g^{\prime}} \quad \Rightarrow \quad \Gamma_{g} \cong \Gamma_{g^{\prime}} .
$$

This follows from the fact that $\Gamma_{g} \cong \Lambda_{\hat{g}}$ and $\Gamma_{g^{\prime}} \cong \Lambda_{\hat{g}^{\prime}}$ by construction, and moreover $\hat{g}$ and $\hat{g}^{\prime}$ are conjugated in $C o_{0}$. However, it can happen that $\Gamma_{g}, \Gamma_{g^{\prime}} \subset \Gamma^{4,20}$ are isomorphic as abstract sublattices, but are not conjugated within $O^{+}\left(\Gamma^{4,20}\right)$. Indeed, as argued in detail in "Appendix D," the problem of determining the number of $O^{+}\left(\Gamma^{4,20}\right)$ conjugacy classes for a given Frame shape can be reduced to that of classifying the (in a suitable sense) inequivalent primitive embeddings of the corresponding lattice $\Lambda_{\hat{g}}$ in $\Gamma^{4,20}$. The proof of this statement can be found in "Appendix D," and the result of the classification is tabulated in "Appendix D.2."

A summary of these results is the following. Out of the 42 distinct four-plane preserving Frame shapes of $O^{+}\left(\Gamma^{4,20}\right)$, there was only one (with Frame shape $1^{-4} 2^{5} 3^{4} 6^{1}$ ) for which we were unable to determine the number of its $O\left(\Gamma^{4,20}\right)$ (and thus $O^{+}\left(\Gamma^{4,20}\right)$ ) classes. For this Frame shape, we can only prove that either a) there is one class or $b$ ) there are two classes for which one is the inverse of the other. The remaining $41 \mathrm{Co}_{0}$ conjugacy classes give rise to 58 distinct $O\left(\Gamma^{4,20}\right)$ conjugacy classes and 80 distinct $O^{+}\left(\Gamma^{4,20}\right)$ conjugacy classes.

\subsection{World-sheet parity}

We have argued earlier that the twining genera $\mathcal{Z}_{g}$ are invariant under conjugation by $\mathrm{O}^{+}\left(\Gamma^{4,20}\right)$ dualities. In many physical applications, however, the larger group $O\left(\Gamma^{4,20}\right)$ is taken to be the relevant duality group. As will be discussed below, the elements of $O\left(\Gamma^{4,20}\right) \backslash O^{+}\left(\Gamma^{4,20}\right)$ correspond to dualities between NLSMs that reverse the world-sheet orientation. In particular, we will show that the twining genera $\mathcal{Z}_{g}$, on the other hand, are in general different unless the two theories are related by an element of $O^{+}\left(\Gamma^{4,20}\right) \subset O\left(\Gamma^{4,20}\right)$. For this reason, it is necessary, for our purposes, to consider two NLSMs related by dualities 
$h \in O\left(\Gamma^{4,20}\right) \backslash O^{+}\left(\Gamma^{4,20}\right)$ as distinct, although we will still refer to such $h$ as (orientationreversing) dualities.

Let us first explain why only elements of $O^{+}\left(\Gamma^{4,20}\right) \subset O\left(\Gamma^{4,20}\right)$, which by definition preserve the orientation of any positive four-plane, preserve the orientation of the worldsheet of NLSM [75]. To understand this, let us first recall some known facts about NLSM on K3 (see, e.g., [75] for a discussion and more details). The tangent space to $(S O(4) \times O(20)) \backslash O^{+}(4,20) \cong(O(4) \times O(20)) \backslash O(4,20)$ at the point corresponding to a four plane $\Pi$ can be identified with the 80 -dimensional space of $\mathcal{N}=(4,4)$-preserving exactly marginal operators of the corresponding $\operatorname{NLSM} \mathcal{T}(\Pi)$. The latter have schematically the form $G_{-1 / 2} \bar{G}_{-1 / 2} \chi_{i}$, where $\chi_{i}, i=1, \ldots, 20$, are fields of weight $(1 / 2,1 / 2)$ belonging to 20 different $\mathcal{N}=(4,4)$ irreducible representations. In particular, the $\mathcal{N}=(4,4)$-preserving marginal operators are the descendants of conformal weights $(1,1)$ that are singlets under the internal R-symmetries $S U(2)_{L}^{\text {susy }} \times S U(2)_{R}^{\text {susy }}$, generated by the zero modes of the holomorphic and anti-holomorphic $\operatorname{si}(2)_{1}$ current algebras within $\mathcal{N}=(4,4)$. On the other hand, they transform as $(2,2)$ under the group $S U(2)_{L}^{\text {out }} \times S U(2)_{R}^{\text {out }}$ of outer automorphisms acting on the left- and right-moving supercharges. The space $(S O(4) \times O(20)) \backslash O^{+}(4,20)$ has an holonomy group $S O(4) \times S O(20)$, where the $S O(4)$ factor is the group $S O(\Pi)$ of rotations of the four-plane $\Pi \subset \mathbb{R}^{4,20}$, while $S O(20)$ rotates its orthogonal complement $\Pi^{\perp} \subset \mathbb{R}^{4,20}$. This holonomy group must act on the space of exactly marginal operators $G_{-1 / 2} \bar{G}_{-1 / 2} \chi_{i}$; in particular, the $S O(20)$ factor rotates the fields $\chi_{i}$, while the $S O(\Pi) \cong S O(4)$ factor acts on the supercharges $G_{-1 / 2}$ and $\bar{G}_{-1 / 2}$ by automorphisms of the $\mathcal{N}=(4,4)$ superconformal algebra. Therefore, the double cover $\operatorname{Spin}(\Pi) \cong \operatorname{Spin}(4)$ of $S O(\Pi)$ can be identified with the group $S U(2)_{L}^{\text {out }} \times S U(2)_{R}^{\text {out }}$ of algebra automorphisms. Now, let $h \in O\left(\Gamma^{4,20}\right)$ be a duality mapping $\mathcal{T}(\Pi)$ to $\mathcal{T}(h(\Pi))$. The group $S O(h(\Pi))$ rotating the dual 4-plane $h(\Pi)$ and its double cover $\operatorname{Spin}(h(\Pi))$ are the conjugate by $h$ of the group $S O(\Pi) \subset O^{+}(4,20)$ rotating $\Pi$ and its cover $\operatorname{Spin}(\Pi) \cong S U(2)_{L}^{\text {out }} \times S U(2)_{R}^{\text {out }}$, respectively. Recall that, given an oriented Euclidean four-dimensional space, conjugation by (a lift of) an element $h \in S O(4)$ maps each $S U(2)$ factor in the spin cover $\operatorname{Spin}(4) \cong S U(2) \times S U(2)$ into itself, while conjugation by $h \in O(4) \backslash S O(4)$ exchanges the two $S U(2)$ factors. Therefore, if a duality $h$ is in $O^{+}\left(\Gamma^{4,20}\right)$, then conjugation by $h$ will send the factor $S U(2)_{L}^{\text {out }}$ (respectively, $S U(2)_{R}^{o u t}$ ) of $\operatorname{Spin}(\Pi)$ to the group of outer automorphisms of the left-moving (resp., right-moving) $\mathcal{N}=4$ superconformal algebra in the dual model $\mathcal{T}(h(\Pi))$. On the other hand, if $h \in O\left(\Gamma^{4,20}\right) \backslash O^{+}\left(\Gamma^{4,20}\right)$, conjugation by $h$ will map the group of leftmoving outer automorphisms $S U(2)_{L}^{\text {out }}$ in the model $\mathcal{T}(\Pi)$ to the group of right-moving outer automorphisms in the dual model $\mathcal{T}(h(\Pi))$, and vice versa. By consistency, in this case $h$ must also send the holomorphic $\mathcal{N}=4$ superconformal algebra in $\mathcal{T}(\Pi)$ to the anti-holomorphic $\mathcal{N}=4$ algebra in $\mathcal{T}(h(\Pi))$. This means that a duality $h \in O\left(\Gamma^{4,20}\right)$ preserves the world-sheet orientation if and only if $h \in O^{+}\left(\Gamma^{4,20}\right) \subset O\left(\Gamma^{4,20}\right)$. Since the definition of the twining genera effectively only focuses on the action of $g$ on the leftmovers (i.e. on the right-moving ground states), and in general $g$ acts on the right-movers differently, one expects $\mathcal{Z}_{g}$ to be invariant only under $O^{+}\left(\Gamma^{4,20}\right)$ duality transformations.

This consideration is particularly relevant for symmetries whose corresponding twining genera have complex multiplier systems. Recall that the twined elliptic genus $\mathcal{Z}_{g}$ is a Jacobi form under a certain congruence subgroup $\mathfrak{G}_{g} \subset S L_{2}(\mathbb{Z})$ with a (in general non-trivial) multiplier $\psi_{g}: \mathfrak{G}_{g} \rightarrow \mathbb{C}^{*}$. We say that $\psi_{g}$ is a complex multiplier system if its image does not lie in $\mathbb{R}$. Note that this is necessarily the case when the multiplier has order greater 
than 2 . To see the relation between world-sheet parity and the multiplier system, consider two $K 3$ NLSMs $\mathcal{T}$ and $\mathcal{T}^{\prime}$ corresponding to the four planes $\Pi$ and $\Pi^{\prime}$ that are related by an $h \in O\left(\Gamma^{4,20}\right), \Pi^{\prime}=h(\Pi)$, which reverses the orientation of a positive four-plane and hence exchanges the left- and the right-movers. This means in particular that $h$ maps the $\mathcal{N}=(4,4)$ algebras of $\mathcal{T}$ and $\mathcal{T}^{\prime}$ as

$$
h L_{n} h^{-1}=\bar{L}_{n}^{\prime} \quad h J_{n} h^{-1}=\bar{J}_{n}^{\prime} .
$$

Given a symmetry $g$ of $\mathcal{T}$, namely $g \in O^{+}\left(\Gamma^{4,20}\right)$ such that $g$ fixes $\Pi$ pointwise, then a corresponding symmetry of $\mathcal{T}^{\prime}$ is given by $g^{\prime}:=h g h^{-1}$. We would like to know whether $\mathcal{Z}_{g}(\mathcal{T} ; \tau, z)$ and $\mathcal{Z}_{g^{\prime}}\left(\mathcal{T}^{\prime} ; \tau, z\right)$ are the same.

To answer this question, consider the refined twining partition function

$$
Z_{g}(\mathcal{T} ; \tau, z, \bar{u})=\operatorname{Tr}_{\mathcal{H}_{R R}}\left(g q^{L_{0}-\frac{c}{24}} \bar{q}^{\bar{L}_{0}-\frac{\bar{c}}{24}} e^{2 \pi i z J_{0}} e^{-2 \pi i \bar{u} \bar{J}_{0}}(-1)^{F+\bar{F}}\right)
$$

for a symmetry $\langle g\rangle$ of the theory $\mathcal{T}$. Note that, unlike the elliptic genus, this function is not an index, and it depends on both the conjugacy class of $g$ and the point in moduli space, $\mathcal{T}$. In general, $Z_{g}$ is not holomorphic in $\tau$, but it is elliptic (one can apply spectral flow independently to the left- and right-movers) and modular (in the appropriate sense for a non-holomorphic Jacobi form) under some subgroup of $S L_{2}(\mathbb{Z})$. In particular, if $g$ has order $N$, we expect $Z_{g}$ to transform under $\left(\begin{array}{ll}a & b \\ c & d\end{array}\right) \in \Gamma_{1}(N)$ as

$$
Z_{g}(\mathcal{T} ; \tau, z, u)=\psi_{g}\left(\left(\begin{array}{ll}
a & b \\
c d
\end{array}\right)\right) e^{-2 \pi i\left(\frac{c c^{2}}{c \tau+d}+\frac{c \bar{u}^{2}}{c \bar{u}+d}\right)} Z_{g}\left(\mathcal{T} ; \frac{a \tau+b}{c \tau+d}, \frac{z}{c \tau+d}, \frac{\bar{u}}{c \bar{\tau}+d}\right) .
$$

Clearly, one recovers the twining genus as

$$
Z_{g}(\mathcal{T} ; \tau, z, \bar{u}=0)=\mathcal{Z}_{g}(\mathcal{T} ; \tau, z) .
$$

This implies that the multiplier $\psi_{g}$ of the twining partition function $Z_{g}$ coincides with the one of the twining genus $\mathcal{Z}_{g}$.

Now, the $O\left(\Gamma^{4,20}\right)$-equivalence and the absence of $O^{+}\left(\Gamma^{4,20}\right)$-equivalence between the theories $\mathcal{T}$ and $\mathcal{T}^{\prime}$ imply

$$
Z_{g^{\prime}}\left(\mathcal{T}^{\prime} ; \tau^{\prime}, z^{\prime}, \bar{u}^{\prime}\right)=Z_{g}(\mathcal{T} ; \tau, z, \bar{u})
$$

In the above, apart from $g^{\prime}=h g h^{-1}$ we also have $\tau^{\prime}=-\bar{\tau}, z^{\prime}=-\bar{u}$ and $\bar{u}^{\prime}=-z$. To see the relation between the multiplier system of $\mathcal{Z}_{g^{\prime}}\left(\mathcal{T}^{\prime}\right)$ and $\psi_{g}$, note that the above equation implies

$$
Z_{g^{\prime}}\left(\mathcal{T}^{\prime} ; \tau, 0, z\right)=Z_{g}(\mathcal{T} ;-\bar{\tau},-\bar{z}, 0)=\mathcal{Z}_{g}(\mathcal{T} ;-\bar{\tau},-\bar{z})
$$

As a result, assuming that the coefficients of the double series expansions in $q$ and $y$ of $\mathcal{Z}_{g}(\mathcal{T})$ are all real, we obtain

$$
Z_{g^{\prime}}\left(\mathcal{T}^{\prime} ; \tau, 0, z\right)=\overline{\mathcal{Z}_{g}(\mathcal{T} ; \tau, z)}
$$

and hence has multiplier given by $\overline{\psi_{g}}: \Gamma_{1}(N) \rightarrow \mathbb{C}^{*}$, the inverse of the multiplier of $\mathcal{Z}_{g}$. The above assumption can be proven from the fact that $\overline{\operatorname{Tr}_{V}(g)}=\operatorname{Tr}_{V}\left(g^{-1}\right)$ for any 
finite-dimensional representation $V$ of a finite group $\langle g\rangle$ and using the identity $\mathcal{Z}_{g}=\mathcal{Z}_{g^{-1}}$ (see eq.(3.7)).

Finally, recall that $Z_{g^{\prime}}\left(\mathcal{T}^{\prime} ; \tau, 0, u\right)$ and $Z_{g^{\prime}}\left(\mathcal{T}^{\prime} ; \tau, z, 0\right)=\mathcal{Z}_{g^{\prime}}\left(\mathcal{T}^{\prime} ; \tau, z\right)$ necessarily have the same multiplier, since they both coincide with that of $Z_{g^{\prime}}\left(\mathcal{T}^{\prime} ; \tau, u, z\right)$, and thus we conclude that the twining genera $\mathcal{Z}_{g^{\prime}}\left(\mathcal{T}^{\prime} ; \tau, z\right)$ and $\mathcal{Z}_{g}(\mathcal{T} ; \tau, z)$ have multiplier systems that are the inverse (equivalently, complex conjugate) of each other. In particular, $\mathcal{Z}_{g^{\prime}}\left(\mathcal{T}^{\prime} ; \tau, z\right)$ and $\mathcal{Z}_{g}(\mathcal{T} ; \tau, z)$ cannot be the same unless $\psi_{g}=\overline{\psi_{g}}$. As a result, symmetries $g$ leading to a twining genus with a complex multiplier system necessarily act differently on left- and right-moving states. Note, however, that it can happen that a symmetry acting asymmetrically on left- and right-movers leads to a twining genus with a multiplier system of order one or two. In what follows, we will refer to a symmetry $g$ of a NLSM a complex symmetry if the resulting twining genus has complex multiplier system.

\subsection{Conway and umbral moonshine}

Once the possible $O^{+}\left(\Gamma^{4,20}\right)$ classes of symmetries have been determined, it remains to calculate the corresponding twining genera. As we will see in Sect. 4, many examples have been computed in specific NLSMs. However, the list of such functions is still incomplete. After reviewing the earlier work [22,42], in this subsection we present two conjectures relating physical twining genera to functions arising from umbral and Conway moonshine, as well as some evidence for their validity.

Consider the 23 Niemeier lattices $N$ with non-trivial root systems. Umbral moonshine attaches to each element $g$ of the Niemeier group $G_{N}$ a weight one mock Jacobi form

$$
\Psi_{g}^{N}(\tau, z)=\sum_{r \in \mathbb{Z} / 2 m} H_{g ; r}^{N}(\tau) \theta_{m, r}(\tau, z)
$$

whose index is given by the Coxeter number of the root system of the corresponding Niemeier lattice $N$ [16]. In the above expression, the index $m$ theta functions are given by

$$
\theta_{m, r}(\tau, z)=\sum_{k=r \bmod 2 m} q^{k^{2} / 4 m} y^{k}
$$

and the vector-valued mock modular form $H_{g}^{N}=\left(H_{g ; r}^{N}\right)$ contains precisely the same information as the mock Jacobi form $\Psi_{g}^{N}$. In [22], a weight 0 index 1 Jacobi form for a certain $\mathfrak{G}_{g} \subseteq S L_{2}(\mathbb{Z})$ is then given in terms of $\Psi_{g}^{N}$ by

$$
\phi_{g}^{N}(\tau, z)=\mathcal{Z}_{g}(N ; \tau, z)+\left.\frac{\theta_{1}^{2}(\tau, z)}{2 \eta^{6}(\tau)}\left(\frac{1}{2 \pi i} \frac{\partial}{\partial w} \Psi_{g}^{N}(\tau, w)\right)\right|_{w=0} .
$$

In the above formula, $\mathcal{Z}_{g}(N ; \tau, z)$ denotes the holomorphic part of the ( $g$-twined) elliptic genus of the singularities corresponding to the root system of $N .^{8}$ In string theory and conformal field theory, it is often useful to consider $K 3$ surfaces that are either smooth or have at worst du Val type surface singularities, i.e. singularities locally of the form $\mathbb{C}^{2} / G$, where $G$ is a finite subgroup of $S U(2)_{\mathbb{C}}$ [37]. Note that, upon including this kind of singular surfaces, the moduli space of hyperkähler structures (with fixed volume) on K3 admits a simple description as (a discrete quotient of) the Grassmannian $O(3,19) / O(3) \times O(19)$,

${ }^{8}$ Note that the holomorphic part of the elliptic genus in question is well defined both from a physical and mathematical point of view. From the physics perspective, the holomorphic part corresponds to the contribution from the discrete part of the spectrum $[1,44,59,89]$. From the mathematical point of view, the holomorphic part corresponds to the holomorphic part of the harmonic Maass form [9]. 
parametrizing positive-definite 3-spaces within the space $\Gamma^{3,19} \otimes \mathbb{R} \cong \mathbb{R}^{3,19}$ (see, e.g., [6]). In particular, surfaces with du Val singularities correspond to the case where the 3-space is orthogonal to a root $v \in \Gamma^{3,19}, v^{2}=-2$, and therefore can be obtained as suitable limits of smooth K3 surfaces. Du Val singularities have an ADE classification, formally analogous to the one of simply laced root systems. A conformal field theory description of string theory with ADE singularities as the target space was given in [79]. The form of their elliptic genus was investigated in a number of papers, including $[1,2,22,44,63,74,89]$. For instance, when $N$ is the Niemeier lattice with root system $24 A_{1}, \mathcal{Z}(N ; \tau, z):=24 \mathcal{Z}\left(A_{1} ; \tau, z\right)$ is 24 times the holomorphic part of the elliptic genus of an $A_{1}$-singularity.

It was conjectured in [22] that $\phi_{g}^{N}$ are candidates for twining genera arising from $K 3$ NLSMs when $g$ preserves a four-plane; this conjecture has passed a few consistency tests and was further tested in [17]. For a given $N$ with a non-trivial root system, we will denote the set of Jacobi forms arising in this way as

$$
\Phi(N):=\left\{\phi_{g}^{N} \mid g \text { is a four-plane preserving element of } G_{N}\right\} .
$$

The construction and conjecture in [22] gives us a set of Jacobi forms $\Phi(N)$ attached to each of the 23 Niemeier lattice $N$ with roots that (conjecturally) play the role of twined $K 3$ elliptic genera at certain points in the moduli space. It is also possible to define a similar set $\Phi(\Lambda)$ associated with the Leech lattice $\Lambda$, though the construction is quite different. In [42] Duncan and Mack-Crane proposed two (possibly coinciding) weight 0 index 1 weak Jacobi forms for a certain $\mathfrak{G}_{g} \subseteq S L_{2}(\mathbb{Z})$, denoted $\phi_{g,+}^{\Lambda}(\tau, z)$ and $\phi_{g,-}^{\Lambda}(\tau, z)$, to each of the four-plane preserving conjugacy classes of $\mathrm{Co}_{0} \cdot{ }^{9}$ The construction of $\phi_{g,+}^{\Lambda}$ and $\phi_{g,-}^{\Lambda}$ is based on an $\mathcal{N}=1$ super VOA of central charge $c=12$, which has symmetry group $\mathrm{Co}_{0}$ [40]. Concretely, one has

$$
\phi_{g \pm \pm}^{\Lambda}(\tau, z)=\sum_{i=1}^{4} \epsilon_{g, i} \theta_{i}^{2}(\tau, z) \prod_{k=1}^{10} \theta_{i}^{2}\left(\tau, \rho_{g, k}\right)
$$

where $\rho_{g, k}$ are such that

$$
\left\{1,1,1,1, e^{-2 \pi i \rho_{g 1}}, e^{2 \pi i \rho_{g, 1}}, \ldots, e^{-2 \pi i \rho_{g, 10}}, e^{2 \pi i \rho_{g, 10}}\right\}
$$

are the 24 eigenvalues of $g$ acting on the 24-dimensional representation, and

$$
\epsilon_{g, i}= \begin{cases}\mp 1 & i=1, \\ -\frac{\operatorname{Tr}_{4096} g}{4 \prod_{k=1}^{10}\left(e^{-\pi i \rho_{g, k}}+e^{\left.\pi i \rho_{g, k}\right)}\right.} & i=2, \\ 1 & i=3 \\ -1 & i=4 .\end{cases}
$$

In the above formula, obtained via a simple manipulation (see [14], eq. (5.10) and the discussion therein) of the expressions given in [42], 4096 is the representation of $\mathrm{Co}_{0}$ that decomposes as $4096=1+276+1771+24+2024$ in terms of $\mathrm{Co}_{0}$ irreducible representations. This representation corresponds to the space of fermionic ground states for the $\mathcal{N}=1$ super VOA in [40].

\footnotetext{
${ }^{9}$ Note that, in [40], the Jacobi form attached to a $\mathrm{Co}_{0}$ conjugacy class with representative $g$ depends on the choice of an orientation for the space $\tilde{H}(X, \mathbb{Z}) \otimes \mathbb{R}$ (see the beginning of section 9 in [40]). In particular, in the explicit formulae for $\phi_{g}$ (eq.(9.14) of [40]), this choice of orientation determines the sign of the parameter $D_{g}$, while its absolute value depends on the $\mathrm{Co}_{0}$ conjugacy class. Therefore, $\phi_{g,+}^{\Lambda}(\tau, z)$ and $\phi_{g,-}^{\Lambda}(\tau, z)$ coincide only for those classes for which $D_{g}=0$. See also [14] for further discussions about this point.
} 
One has $\phi_{g,+}^{\Lambda} \neq \phi_{g,-}^{\Lambda}$ if and only if the invariant sublattice $\Lambda^{g}$ has exactly rank four. Henceforth, we define

$$
\Phi(\Lambda):=\left\{\phi_{g,+}^{\Lambda}, \phi_{g,-}^{\Lambda} \mid g \text { is a four-plane preserving element of } \mathrm{Co}_{0}\right\} .
$$

The authors of [42] then conjectured that the functions in $\Phi(\Lambda)$ are relevant for twining genera arising from (non-singular) $K 3$ NLSMs. In fact, they conjecture that all twining genera arising from any $K 3$ NLSM coincide with some element of $\Phi(\Lambda)$ arising from the Conway module, which is supported by the non-trivial fact that all the known twining genera $\mathcal{Z}_{g}$ coincide with a function in $\Phi(\Lambda)$.

There are a few motivations for us to modify this conjecture and to make the conjecture in [22] more concrete. Firstly, the classification theorems of Sect. 2 suggest that if one does not exclude the loci in the moduli space (2.2) corresponding to singular four planes, one should treat the Leech lattice and the other 23 Niemeier lattices with non-trivial root systems on an equal footing when discussing the four-plane preserving symmetry groups. As a consequence, one might expect both Conway and umbral moonshine to play a role in describing the twining genera. Secondly, UV descriptions of K3 NLSMs given by LandauGinzburg (LG) orbifolds furnish evidence that suggests that the Conway functions alone are not sufficient to capture all the twining genera [17] (see also Sect. 4.3). To be more precise, there are twining genera arising from symmetries of $U V$ theories that flow to $K 3$ NLSMs in the IR that can be reproduced from the set $\Phi(N)$ for some $N$ with roots, but do not coincide with anything in $\Phi(\Lambda)$. One caveat preventing this result from being a definitive argument is that the action of the corresponding symmetry on the $\operatorname{IR} \mathcal{N}=(4,4)$ superconformal algebra is not accessible in the UV analysis.

The third and arguably most convincing argument to include functions arising from both Conway and umbral moonshine is the following. As we have seen in Sect. 3.2, a pair of theories related by a flip of world-sheet parity gives rise to twining genera with inverse multiplier systems. At the same time, $\Phi(\Lambda)$ contains some twining functions with a complex multiplier system and no functions with the inverse multiplier. Such functions can always be recovered from $\Phi(N)$ for some other Niemeier lattice $N$. As a result, no single $\Phi(N)$ (not even for $N$ the Leech lattice) is sufficient to reproduce both a physical twining function $\mathcal{Z}_{g}(\mathcal{T})$ with complex multiplier and its parity-flipped counterpart $\mathcal{Z}_{g^{\prime}}\left(\mathcal{T}^{\prime}\right)$.

These observations lead us to formulate the following conjecture:

Conjecture 5 Let $\mathcal{T}(\Pi)$ be a $K 3$ NLSM, and let $G$ be its symmetry group. Then, there exists at least one Niemeier lattice $N$ such that $\Gamma_{G}$ can be embedded in $N, G \subseteq G_{N}$, and for any $g \in G$ the twining genus $\mathcal{Z}_{g}$ coincides with an element of $\Phi(N)$.

In other words, we conjecture that for each $K 3 \operatorname{NLSM} \mathcal{T}$, the set $\tilde{\Phi}(\mathcal{T}):=\left\{\mathcal{Z}_{g}(\mathcal{T}(\Pi)) \mid g \in\right.$ $O^{+}\left(\Gamma^{4,20}\right), g$ fixes $\Pi$ pointwise $\}$ of physical twining genera is a subset of the $\Phi(N)$ for some Niemeier lattice $N$. Clearly, for most theories, the Niemeier lattice $N$ satisfying the above properties is not unique. In particular, recall that there are many coincidences among the functions associated with different Niemeier lattices. In other words, there exist $\phi \in \Phi(N), \phi^{\prime} \in \Phi\left(N^{\prime}\right)$ with $N \neq N^{\prime}$ such that $\phi=\phi^{\prime}$.

Conversely, we conjecture that all elements of $\Phi(N)$ play a role in capturing the symmetries of BPS states of $K 3$ NLSMs:

Conjecture 6 For any element $\phi$ of any of the $24 \Phi(N)$, there exists a NLSM $\mathcal{T}$ with a symmetryg such that $\phi=\mathcal{Z}_{g}(\mathcal{T})$. 
In Sect. 4.5, we collect some evidence supporting these conjectures. We will close this section with a few remarks on the consequences of the above conjectures, in relation to the complex symmetries discussed in Sect. 3.2.

- If a given function in $\Phi(N)$ has complex multiplier system, then Conjecture 6 implies that it has to coincide with a twining genus arising from a complex symmetry acting differently on the left- and right-moving Hilbert spaces.

- As we argued in Sect. 3.2, if a theory $\mathcal{T}$ leads to the twining function $\mathcal{Z}_{g}(\mathcal{T})$ with a complex multiplier system, the parity-flipped theory $\mathcal{T}^{\prime}$ has a twining genus $\mathcal{Z}_{g^{\prime}}\left(\mathcal{T}^{\prime}\right)$ with the inverse multiplier system. As a result, the following observations constitute consistency checks and circumstantial evidence for Conjecture 5 and Conjecture 6. Namely, whenever there exist a Niemeier lattice $N$ and a function $\phi \in \Phi(N)$ with a complex multiplier system, arising from a group element with a given Frame shape $\pi$, then there exists at least one other Niemeier lattice $N^{\prime}$ such that there exists a $\phi^{\prime} \in \Phi\left(N^{\prime}\right)$ with the inverse complex multiplier system, which moreover arises from a group element with the same Frame shape $\pi$. See Table 3 for the pairs $\left(N^{\prime}, g^{\prime}\right)$ with the above properties.

- In fact, by inspection one can check that there are never two functions $\phi, \phi^{\prime} \in \Phi(N)$ arising from the same Niemeier lattice that have inverse complex multiplier systems. As a result, Conjecture 5 predicts that a theory corresponding to the four-plane $\Pi$ must have its orthogonal sublattice $\Gamma^{4,20} \cap \Pi^{\perp}$ embeddable into more than one Niemeier lattice in the event that it has a complex symmetry.

- Recall that a theory in the NLSM moduli space (2.2) on a torus orbifold locus-one of the few types of exactly solvable models-always contains symmetries which can only be embedded using the Leech lattice (in the sense of Theorem 1) [51]. As a result, assuming the veracity of Conjecture 5 , complex symmetries can never arise in such a model. This makes it particularly difficult to find examples of K3 NLSMs with complex symmetries and probably explains why we have seen no such examples so far. In Sect. 4.3, we will discuss results of the aforementioned investigation of LG orbifolds [17], while in Sect. 4.4 we will analyze the constraints on such genera coming from modularity.

\section{Examples}

In this section, we collect all known explicit calculations of twining genera in NLSMs on $K 3$. Most of these results have appeared earlier in the literature, the only exceptions being certain genera appearing in Sects. 4.2 and 4.4. See Table 4 for the data. While these examples do not cover the complete set of all possible twining genera, the fact that these partial results fit nicely with the general properties described in the previous sections represents strong evidence in favor of our conjectures.

\subsection{Geometric symmetries}

We say that a symmetry of a $K 3$ NLSM is a geometric symmetry if it is induced from a hyper-Kähler preserving automorphism of the target $K 3$ surface. These symmetries are characterized by the property that the fixed sublattice $\Gamma^{g}$ contains a unimodular $\Gamma^{1,1}$, which can be interpreted as the components $H_{0}(S, \mathbb{Z}) \oplus H_{4}(S, \mathbb{Z})$ of degree 0 and 4 in the 
integral homology of the $K 3$ surface $S$. There exist such geometric symmetries with order $N \in\{2,3,4,5,6,7,8\}[73,77]$. For each of these orders, there is precisely one Frame shape

$$
1^{8} 2^{8}, \quad 1^{6} 3^{6}, \quad 1^{4} 2^{4} 4^{4}, \quad 1^{4} 5^{4}, \quad 1^{2} 2^{2} 3^{2} 6^{2}, \quad 1^{3} 7^{3}, \quad 1^{2} 2^{1} 4^{1} 8^{2}
$$

that can arise from a geometric symmetry of a $K 3$ surface [73].

A general formula for the corresponding twining genus for each of the above Frame shapes and reads

$$
\mathcal{Z}_{g}(\tau, z)=\frac{\operatorname{Tr}_{\mathbf{2 4}}(g)}{\varphi(N)} \sum_{\substack{n \in \mathbb{Z} / N \mathbb{Z} \\ g c d(n, N)=1}} \frac{\vartheta_{1}\left(\tau, z+\frac{n}{N}\right) \vartheta_{1}\left(\tau, z-\frac{n}{N}\right)}{\vartheta_{1}\left(\tau, \frac{n}{N}\right) \vartheta_{1}\left(\tau,-\frac{n}{N}\right)},
$$

where the totient function $\varphi(N):=\left|(\mathbb{Z} / N \mathbb{Z})^{\times}\right|$is number of integers $\bmod N$ that are coprime to $N$. This formula follows from appendix A of [82]; a slightly simplified version valid for $N=2,3,4,6$ appears in [19]. These twining genera can be defined in purely geometric terms as equivariant complex elliptic genera and computed using a version of the Lefschetz fixed-point formula [27]. The results agree with the formulas derived from NLSMs.

\subsection{Torus orbifolds}

If a $K 3$ model is obtained as a (possibly asymmetric) orbifold of a torus $T^{4}$ by a symmetry $g$ of order $N$, then it has a quantum symmetry $Q$ of order $N$, which acts as multiplication by $e^{\frac{2 \pi i r}{N}}$ on all states in the $g^{r}$-twisted sector, $r \in \mathbb{Z} / N \mathbb{Z}$. It is not difficult to compute the twining genus of a quantum symmetry, since it can be computed from the twining genus of $g$ on the NLSM $\mathcal{T}_{T^{4}}$ on the $T^{4}$. In [90], general formulas for the twining genera of all possible symmetries of any NLSM on $T^{4}$ were given. The supersymmetric NLSM on $T^{4}$ has four left-moving and four right-moving Majorana-Weyl fermions. The holomorphic fermions form two doublets $\left(\chi_{i}^{+}, \chi_{i}^{-}\right), i=1,2$, each in the $(2,1)$ representation of the $S U(2)_{L} \times S U(2)_{R}$ R-symmetry, while the anti-holomorphic fermions form doublets $\left(\tilde{\chi}_{i}^{+}, \tilde{\chi}_{i}^{-}\right), i=1,2$, in the $(\mathbf{1}, 2)$ representation. The symmetry $g$ commutes with the R-symmetry and acts on the multiplets by

$$
\begin{array}{rlrl}
\left(\chi_{1}^{+}, \chi_{1}^{-}\right) & \mapsto \zeta_{L}\left(\chi_{1}^{+}, \chi_{1}^{-}\right), & \left(\chi_{2}^{+}, \chi_{2}^{-}\right) \mapsto \zeta_{L}^{-1}\left(\chi_{2}^{+}, \chi_{2}^{-}\right), \\
\left(\tilde{\chi}_{1}^{+}, \tilde{\chi}_{1}^{-}\right) \mapsto \zeta_{R}\left(\tilde{\chi}_{1}^{+}, \tilde{\chi}_{1}^{-}\right), & \left(\tilde{\chi}_{2}^{+}, \tilde{\chi}_{2}^{-}\right) \mapsto \zeta_{R}^{-1}\left(\tilde{\chi}_{2}^{+}, \tilde{\chi}_{2}^{-}\right),
\end{array}
$$

with

$$
\zeta_{L, R}=\exp \left(2 \pi i r_{L, R}\right) \quad \text { for some } \quad r_{L}, r_{R} \in \frac{1}{N} \mathbb{Z} / \mathbb{Z} .
$$

For $\zeta_{L} \neq 1$, the twining genus of $g$ is given by

$$
\mathcal{Z}_{g}\left(\mathcal{T}_{T^{4}} ; \tau, z\right)=\left(\zeta_{L}+\zeta_{L}^{-1}-2\right)\left(\zeta_{R}+\zeta_{R}^{-1}-2\right) \frac{\vartheta_{1}\left(\tau, z+r_{L}\right) \vartheta_{1}\left(\tau, z-r_{L}\right)}{\vartheta_{1}\left(\tau, r_{L}\right) \vartheta_{1}\left(\tau, r_{L}\right)} .
$$

Note that the above function is invariant under both $r_{L} \rightarrow-r_{L}$ and $r_{R} \rightarrow-r_{R}$, but is in general not invariant under $r_{L} \leftrightarrow r_{R}$. When $r_{L}=0 \bmod \mathbb{Z}$ (i.e., $\zeta_{L}=1$ ), the twining genus is given instead by 


$$
\mathcal{Z}_{g}\left(\mathcal{T}_{T^{4}} ; \tau, z\right)=\left(\zeta_{R}+\zeta_{R}^{-1}-2\right) \frac{\vartheta_{1}(\tau, z)^{2}}{\eta(\tau)^{6}} \Theta_{L}(\tau)
$$

where

$$
\Theta_{L}(\tau):=\sum_{\lambda \in L} q^{\frac{\lambda^{2}}{2}}
$$

is the theta series associated with a lattice $L$ of rank 4 . The only relevant cases are $\left(r_{L}, r_{R}\right)=$ $(0,1 / 2)$ and $\left(r_{L}, r_{R}\right)=(0,1 / 3)$, in which cases $L$ is the $D_{4}$ or $A_{2}^{2}$ root lattices, respectively (see [90] for more details). In particular, the untwined elliptic genus of $T^{4}$ is $\mathcal{Z}_{e}\left(T^{4} ; \tau, z\right)=0$.

When a CFT has a discrete symmetry, it is also useful to discuss the twisted sectors of the symmetry (modules of the invariant subalgebra), labeled by the twisting group element $g$. For any element $h$ of the discrete symmetry group that commutes with the twisting element $g$, one can consider the graded trace of $h$ over the $g$-twisted sector, analogous to the way in which a twined partition function or twined elliptic genus is defined. Such a character is often called the twisted-twining partition function/elliptic genus. As usual in the literature, we use $\mathcal{Z}_{h, g}$ to denote the $g$-twining function in the $h$-twisted sector. In particular, the twining function of the original unorbifolded theory is given by $\mathcal{Z}_{g}:=\mathcal{Z}_{e, g}$.

Using the modular properties of the theta function as well as the fact that the twistedtwining genera form a representation of $S L_{2}(\mathbb{Z})$, we obtain the following expression which is valid for $r_{L} M \neq 0 \bmod \mathbb{Z}$

$$
\begin{aligned}
\mathcal{Z}_{g^{n}, g^{m}}\left(\mathcal{T}_{T^{4}} ; \tau, z\right)= & \left(\zeta_{L}^{M}+\zeta_{L}^{-M}-2\right)\left(\zeta_{R}^{M}+\zeta_{R}^{-M}-2\right) \\
& \times \frac{\vartheta_{1}\left(\tau, z+r_{L}(n \tau+m)\right) \vartheta_{1}\left(\tau, z-r_{L}(n \tau+m)\right)}{\vartheta_{1}\left(\tau, r_{L}(n \tau+m)\right) \vartheta_{1}\left(\tau, r_{L}(n \tau+m)\right)},
\end{aligned}
$$

where $M=\operatorname{gcd}(n, m)$. When $r_{L} M=0 \bmod \mathbb{Z}, \mathcal{Z}_{g^{n}, g^{m}}$ is given by a suitable $S L_{2}(\mathbb{Z})$ transformation of (4.7).

The elliptic genus of the $g$-orbifolded theory $\mathcal{T}_{K 3}$, which we assume to be a $K 3$ model, is then given in the usual way by

$$
\mathcal{Z}\left(\mathcal{T}_{K 3} ; \tau, z\right)=\frac{1}{N} \sum_{n, m \in \mathbb{Z} / N \mathbb{Z}} \mathcal{Z}_{g^{n}, g^{m}}\left(\mathcal{T}_{T^{4}} ; \tau, z\right)
$$

Similarly, the twining genus of the quantum symmetry $Q$ is given by

$$
\mathcal{Z}_{Q}\left(\mathcal{T}_{K 3} ; \tau, z\right)=\frac{1}{N} \sum_{n, m \in \mathbb{Z} / N \mathbb{Z}} e^{\frac{2 \pi i n}{N}} \mathcal{Z}_{g^{n}, g^{m}}\left(\mathcal{T}_{T^{4}} ; \tau, z\right) .
$$

A number of new twining genera can be obtained from the above calculation. The relevant values of $r_{L}, r_{R}$ and the Frame shapes of the corresponding quantum symmetries are collected in Table 1 (see [90]).

A set of more general twining genera can be obtained as follows. Suppose that $g$ is a symmetry of a NLSM on $T^{4}$ of order $N$ and the $g^{n}$-orbifolded theory is a $K 3$ NLSM for a $n \mid N$. Then, $g$ induces a symmetry $g^{\prime}$ of order $N / n$ on the resulting K3 NLSM that commutes with the quantum symmetry, and one has

$$
\mathcal{Z}_{g^{\prime l} Q^{m}}\left(\mathcal{T}_{K 3} ; \tau, z\right)=\frac{n}{N} \sum_{j, k=1}^{N / n} e^{\frac{2 \pi i j m}{N / n}} \mathcal{Z}_{g^{n j}, g^{n k+l}}\left(\mathcal{T}_{T^{4}} ; \tau, z\right)
$$


Table 1 Frame shapes corresponding to quantum symmetries $Q$ of torus orbifolds

\begin{tabular}{llll}
\hline$r_{L}$ & $r_{R}$ & $\pi_{Q}$ & W-s parity \\
\hline $1 / 2$ & $1 / 2$ & $1^{-8} 2^{16}$ & $\circ$ \\
$1 / 3$ & $1 / 3$ & $1^{-3} 3^{9}$ & $\circ$ \\
$1 / 4$ & $1 / 4$ & $1^{-4} 2^{6} 4^{4}$ & $\circ$ \\
$1 / 6$ & $1 / 6$ & $1^{-4} 2^{5} 3^{4} 6^{1}$ & $\circ$ \\
$1 / 5$ & $2 / 5$ & $1^{-1} 5^{5}$ & $\uparrow$ \\
$2 / 5$ & $1 / 5$ & & $\uparrow$ \\
$1 / 4$ & $1 / 2$ & $2^{-4} 4^{8}$ & \\
$1 / 2$ & $1 / 4$ & & $\uparrow$ \\
$1 / 6$ & $1 / 2$ & $1^{-2} 2^{4} 3^{-2} 6^{4}$ & \\
$1 / 2$ & $1 / 6$ & & \\
$1 / 6$ & $1 / 3$ & $1^{-1} 2^{-1} 3^{3} 6^{3}$ & \\
$1 / 3$ & $1 / 6$ & & \\
$1 / 8$ & $5 / 8$ & $1^{-2} 2^{3} 4^{1} 8^{2}$ & \\
$5 / 8$ & $1 / 8$ & & $\uparrow$ \\
$1 / 10$ & $3 / 10$ & $1^{-2} 2^{3} 5^{2} 10^{1}$ & \\
$3 / 10$ & $1 / 10$ & & $1-22^{2} 3^{2} 4^{1} 12^{1}$ \\
$1 / 12$ & $5 / 12$ & & \\
$5 / 12$ & $1 / 12$ & & \\
\hline
\end{tabular}

The twining genera can be obtained by applying formulae (4.11) and (4.9). In the last column, the symbol o means that the symmetry is fixed by world-sheet parity, while $\uparrow$ that world-sheet parity relates two different symmetries. Clearly, a twining genus for a quantum symmetry is fixed by world-sheet parity if and only if $r_{L}= \pm r_{R} \bmod \mathbb{Z}$

The right-hand side of this equation can be easily computed using (4.9). The Frame shapes corresponding to these symmetries are collected in Table 2.

\subsection{Landau-Ginzburg orbifolds}

It is very non-generic for a $K 3$ NLSM to correspond to an exactly solvable CFT. In fact, the only such examples we know of are torus orbifolds, described in the previous subsection, Gepner models, i.e. orbifolds of tensor products of $\mathcal{N}=2$ minimal models [57], and generalizations thereof [48]. However, for the purpose of computing the (twined) elliptic genus, it is sufficient to have a UV description which flows in the IR to a K3 NLSM. This fact was used by Witten to provide evidence for the connection between certain LandauGinzburg (LG) models and $\mathcal{N}=2$ minimal models [94]. The LG theories are generically massive, super-renormalizable $\mathcal{N}=2$ quantum field theories; however, in the IR they can flow to an $\mathcal{N}=(2,2)$ superconformal field theory. For instance, the LG theory of a single chiral superfield with superpotential

$$
W_{A_{k+1}}(\Phi)=\frac{1}{k+2} \Phi^{k+2}
$$

flows to an IR fixed point corresponding to the $\mathcal{N}=2$ minimal model of type $A_{k+1}$.

Though these minimal models all have central charge less than 3, LG theories prove to have geometric applications through the orbifold construction. Namely, one can construct theories which flow in the IR to a NLSM on a CY $d$-fold by taking superpotentials of multiple chiral multiplets, such that the sum of their charges equals $3 d$, along with an orbifold which projects the Hilbert space onto states with integer $U(1)$ charges. This connection between CY geometry and LG orbifolds was further elucidated by Witten [93] using the framework of gauged linear sigma models. 
Table 2 Symmetries of torus orbifolds whose twining genera are given by (4.12)

\begin{tabular}{lllll}
\hline$r_{L}$ & $r_{R}$ & $o(Q)$ & $o\left(g^{\prime}\right)$ & $\pi_{Q g^{\prime}}$ \\
\hline $1 / 4$ & $1 / 4$ & 2 & 2 & $2^{12}$ \\
$1 / 6$ & $1 / 6$ & 2 & 3 & $1^{4} 2^{1} 3^{-4} 6^{5}$ \\
$1 / 6$ & 3 & 2 & $1^{5} 2^{-4} 3^{1} 6^{4}$ \\
$1 / 6$ & $1 / 2$ & 2 & 3 & $1^{-2} 2^{4} 3^{-2} 6^{4}$ \\
$1 / 8$ & $1 / 3$ & 3 & 2 & $1^{-1} 2^{-1} 3^{3} 6^{3}$ \\
& $3 / 8$ & 2 & 4 & $2^{4} 4^{4}$ \\
$1 / 10$ & $3 / 10$ & 2 & 2 & $2^{4} 4^{4}$ \\
& & 5 & 5 & $1^{2} 2^{1} 5^{-2} 10^{3}$ \\
$1 / 12$ & $5 / 12$ & 6 & 2 & $1^{3} 2^{-2} 5^{1} 10^{2}$ \\
& & 4 & 2 & $2^{3} 6^{3}$ \\
& & 3 & 3 & $1^{2} 3^{-2} 4^{1} 6^{2} 12^{1}$ \\
& & 2 & 4 & $1^{1} 2^{2} 3^{1} 4^{-2} 12^{2}$ \\
\hline
\end{tabular}

In [17], a number of new twinings were found in explicit models: LG orbifolds which flow in the IR to K3 CFTs. Here, we briefly mention cases where symmetries of order 11, 14 and 15 arise. These symmetries preserve precisely a four-plane in the Leech lattice and thus only occur at isolated, non-singular points in $K 3$ moduli space. The symmetries of order 11 and 15 arise in cubic superpotentials of six chiral superfields of the form,

$$
\begin{aligned}
& \mathcal{W}_{1}^{c}(\Phi)=\Phi_{0}^{3}+\Phi_{1}^{2} \Phi_{5}+\Phi_{2}^{2} \Phi_{4}+\Phi_{3}^{2} \Phi_{2}+\Phi_{4}^{2} \Phi_{1}+\Phi_{5}^{2} \Phi_{3} \\
& \mathcal{W}_{2}^{c}(\Phi)=\Phi_{0}^{2} \Phi_{1}+\Phi_{1}^{2} \Phi_{2}+\Phi_{2}^{2} \Phi_{3}+\Phi_{3}^{2} \Phi_{0}+\Phi_{4}^{3}+\Phi_{5}^{3}
\end{aligned}
$$

respectively, while the symmetry of order 14 arises in a model with quartic superpotential

$$
\mathcal{W}^{q}(\Phi)=\Phi_{1}^{3} \Phi_{2}+\Phi_{2}^{3} \Phi_{3}+\Phi_{3}^{3} \Phi_{1}+\Phi_{4}^{4}
$$

As discussed in [17], the symmetry groups of $\mathcal{W}_{1}^{c}, \mathcal{W}_{2}^{c}$, and $\mathcal{W}^{q}$ are given by $L_{2}(11)$, $\left(3 \times A_{5}\right): 2$ and $L_{2}(7) \times 2$, each of which contains elements of order 11, 15 and 14, respectively. Using their explicit actions on the superfields, one can readily compute their LG twining genus.

The symmetries of order 11,15 and 14 all have a unique Frame shape $\left(1^{2} 11^{2}, 1.3 .5 .15\right.$ and 1.2.7.14, respectively), and each occurs in two non-Conway Niemeier groups, corresponding to Niemeier lattices $N_{1}, N_{2}$ with root lattices $\left\{A_{1}^{24}, A_{2}^{12}\right\},\left\{A_{1}^{24}, D_{4}^{6}\right\}$ and $\left\{A_{1}^{24}, A_{3}^{8}\right\}$, respectively. Since these symmetries preserve exactly a four-plane, the Conway module associates two different twinings functions with these Frame shapes. In each of these three cases, the two umbral moonshine twinings given corresponding to two Niemeier lattices yield two different results $\phi_{g_{1}}^{N_{1}}$ and $\phi_{g_{2}}^{N_{2}}$, coinciding with the two twinings $\phi_{g,+}^{\Lambda}$ and $\phi_{g,-}^{\Lambda}$ arising from Conway module.

The twinings of order 11,15 and 14 computed in the above-mentioned LG models match those associated with root systems $A_{2}^{12}, D_{4}^{6}$ and $A_{3}^{8}$, respectively. This can be viewed as evidence for the connection between (non- $M_{24}$ instances of) umbral moonshine and Conway moonshine, to the symmetries of $K 3$ NLSMs. ${ }^{10}$ We refer to [17] for more examples and details.

${ }^{10}$ It is intriguing to note that the forms of $\mathcal{W}_{1}^{c}, \mathcal{W}_{2}^{c}$ and $\mathcal{W}^{q}$ are closely related to the superpotentials which flow to the $A_{2}$, $D_{4}$ and $A_{3} \mathcal{N}=2$ minimal models, where the $A$-type case is given above, and the $D_{4}$ case is $W_{D_{4}}\left(\Phi_{1}, \Phi_{2}\right) \sim \Phi_{1}^{3}+\Phi_{1} \Phi_{2}^{2}$. 


\subsection{Modularity}

In this section, we discuss how one can use constraints of modularity to precisely specify the twining genera corresponding to certain $\mathrm{O}^{+}\left(\Gamma^{4,20}\right)$ conjugacy classes in some cases. The twining genera $\mathcal{Z}_{g}$ are weak Jacobi forms under some congruence subgroup $\mathfrak{G}_{g} \subseteq S L_{2}(\mathbb{Z})$, possibly with a non-trivial multiplier $\psi$. At the same time, the Frame shape establishes the $q^{0}$-terms in their Fourier expansion, given by

$$
\mathcal{Z}_{g}(\tau, z)=2 y+2 y^{-1}+\operatorname{Tr}_{V_{24}}(g)-4+O(q)
$$

Here, $\operatorname{Tr}_{V_{24}}(g)$ denotes the trace of $g \in O^{+}\left(\Gamma^{4,20}\right)$ in the defining 24-dimensional representation $V_{24}$. In some cases, the modular properties together with the above leading-term coefficients are sufficient to fix the function $\mathcal{Z}_{g}$ completely. More precisely, the above criteria dictate that $\mathcal{Z}_{g}$ can be written as

$$
\mathcal{Z}_{g}(\tau, z)=\frac{\operatorname{Tr}_{V_{24}}(g)}{12} \phi_{0,1}(\tau, z)+F(\tau) \phi_{-2,1}(\tau, z)
$$

where

$$
\begin{aligned}
\phi_{0,1}(\tau, z) & =4 \sum_{i=2}^{4} \frac{\theta_{i}(\tau, z)^{2}}{\theta_{i}(\tau, 0)^{2}}=y+10+y^{-1}+O(q) \\
\phi_{-2,1}(\tau, z) & =\frac{\theta_{1}(\tau, z)^{2}}{\eta(\tau)^{6}}=y-2+y^{-1}+O(q),
\end{aligned}
$$

are the standard weak Jacobi forms of index 1 and weight 0 and -2 , respectively, and

$$
F(\tau)=2-\frac{\operatorname{Tr}_{24}(g)}{12}+O(q),
$$

is a modular form of weight 2 under $\mathfrak{G}_{g}$, with a suitable multiplier $\psi$. Clearly, $\psi$ can only be non-trivial when $\operatorname{Tr}_{24}(g)=0$. Let us denote by $M_{2}\left(\mathfrak{G}_{g} ; \psi\right)$ the space of modular forms of weight 2 for a group $\mathfrak{G} \in S L_{2}(\mathbb{Z})$ with multiplier $\psi$. It is clear from (4.17) and (4.21) that $\mathcal{Z}_{g}$ is uniquely determined in terms of $\operatorname{Tr}_{\mathbf{2 4}}(g)$ whenever $\operatorname{dim} M_{2}\left(\mathfrak{G}_{g} ; \psi\right) \leq 1$.

The approach described above is particularly effective in constraining twining genera with non-trivial multiplier $\psi$, since the space $M_{2}\left(\mathfrak{G}_{g} ; \psi\right)$ is often quite small. We illustrate our arguments with the following example. Consider $g$ with Frame shape $3^{8}$. The possible multipliers can be determined using the methods described in "Appendix C." In particular, $\operatorname{Tr}_{V_{24}}(g)=0$ and $\mathfrak{G}_{g}=\Gamma_{0}(3)$, and hence the order of the multiplier system is either 1 or 3. The Witten index of a putative orbifold by $g$ is 8 , which is different from 0 or 24 . We can therefore conclude that the orbifold is inconsistent and hence the multiplier has order $n=3$. (See "Appendix C" for the detailed argument.) Thus, $F(\tau)=2+O(q)$ is modular form of weight 2 for $\Gamma_{0}(3)$ with multiplier of order 3 . It turns out that there are two possible multipliers $\psi$ and $\bar{\psi}$ of order 3 , with the property $\operatorname{dim} M_{2}\left(\Gamma_{0}(3) ; \psi\right)=$ $\operatorname{dim} M_{2}\left(\Gamma_{0}(3) ; \bar{\psi}\right)=1$. Hence, in both cases there are a unique weight 2 form $F$ and therefore a unique weak Jacobi form $\mathcal{Z}_{g}$, with the required normalization (4.21), giving the umbral twining function corresponding to the root systems $A_{1}^{24}$ and $A_{2}^{12}$. 
Using similar arguments, one can determine the twining genera for the Frame shape $4^{6}$ for both possible choices of multipliers, and the twining genera for the Frame shapes $6^{4}$ and $4^{2} 8^{2}$ for one of the two possible multipliers. In all such cases, the resulting twining genera coincide with some umbral functions, i.e., some $\Phi(N)$ (see "Appendix D.2"), offering support for our Conjecture 5.

\subsection{Evidence for the conjectures}

In this section, we summarize a number of results which we view as compelling evidence for our conjectures of Sect. 3.3. Conjecture 5 states, among other things, that all physical twining genera $\mathcal{Z}_{g}$ are reproduced by some function arising from umbral or/and Conway module. If true, then combined with the world-sheet parity analysis in Sect. 3.2, the following two statements necessarily hold. The fact that they do hold then constitutes non-trivial evidence for the conjecture.

- As reported in Appendix D.2, there are either 81 or 82 distinct $O^{+}\left(\Gamma^{4,20}\right)$ classes of symmetries. In particular, for the Frame shape $1^{-4} 2^{5} 3^{4} 6^{1}$, there is either a single $\mathrm{O}^{+}\left(\Gamma^{4,20}\right)$ class or two classes that are the inverse of each other and hence must have the same twining genus. Therefore, there are potentially 81 distinct twining genera $\mathcal{Z}_{g}$. Only 56 have been computed using the methods described in Sects. 4.1-4.4. In all such cases, one has $\mathcal{Z}_{g} \in \Phi(N)$ for at least one Niemeier lattice $N$.

- Whenever there is an umbral or Conway twining genus $\phi_{g} \in \Phi(N)$ which has a complex multiplier $\psi$, there exists another $\phi_{g^{\prime}}^{\prime} \in \Phi\left(N^{\prime}\right)$ corresponding to the same Frame shape $\pi_{g}=\pi_{g^{\prime}}$ and with the conjugate multiplier $\bar{\psi}$. Furthermore, $\pi_{g}$ has distinct $O^{+}\left(\Gamma^{4,20}\right)$ conjugacy classes which are related by world-sheet parity. Note that in all cases we have $N \neq N^{\prime}$. Table 3 shows the pairs of $N, N^{\prime}$, denoted in terms of their root systems in the case $N \neq \Lambda$, leading to Jacobi forms with complex conjugate multipliers.

Similarly, the following fact is non-trivially compatible with Conjecture 6.

- Fix a four-plane preserving Frame shape $\pi_{g}$. Denote by $K$ the number of distinct twining functions $\phi_{g}^{N}$ associated with $\pi_{g}$ arising from either Conway or umbral moonshine, and denote by $K^{\prime}$ the number of $O^{+}\left(\Gamma^{4,20}\right)$ conjugacy classes associated with $\pi_{g}$. In all cases, $K^{\prime} \geq K$, and for a vast majority (35 out of 42) of the four-plane preserving Frame shapes this inequality is saturated.

Note that the fact that $K$ is small can be attributed to the large number of coincidences among the elements of $\Phi(N)$ and $\Phi\left(N^{\prime}\right)$ related to different Niemeier lattices $N, N^{\prime}$. For example, the Frame shape $2^{4} 4^{4}$ appears in the group $G_{N}$ for seven distinct Niemeier lattices $N$, but the seven twining genera $\phi_{g}^{N}$ are all the same, compatible with the fact that there is a unique $O^{+}\left(\Gamma^{4,20}\right)$ class for this Frame shape. Since for some (7 out of 42) Frame shapes the number of $O^{+}\left(\Gamma^{4,20}\right)$-classes is strictly larger than the number of distinct $\phi_{g}^{N}$, Conjecture 5 predicts that there must be some coincidences among the physical twining genera corresponding to these different classes. 
Table 3 Frame shapes with complex multiplier and corresponding Niemeier lattices

\begin{tabular}{lll}
\hline Frame shape & $\psi$ & $\bar{\psi}$ \\
\hline $3^{8}$ & $A_{2}^{12}, D_{4}^{6}, A_{8}^{3}, E_{8}^{3}, \Lambda$ & $A_{1}^{24}, A_{4}^{6}, D_{8}^{3}$ \\
$4^{6}$ & $A_{3}^{8}, A_{1}^{6}, A_{1}^{2}, \Lambda$ & $A_{1}^{24}, A_{2}^{12}, A_{6}^{4}, D_{6}^{4}$ \\
$6^{4}$ & $A_{2}^{12}, D_{4}^{6}, A_{8}^{3}, \Lambda$ & $A_{1}^{24}, A_{4}^{6}$ \\
$4^{2} 8^{2}$ & $A_{3}^{8}, E_{6}^{4}, \Lambda$ & $A_{2}^{12}$ \\
\hline
\end{tabular}

\section{Discussion}

In the present paper, we have proven classification results on lattices and groups relevant for symmetries of $K 3$ string theory and proposed conjectures regarding the relation between the these symmetries and umbral and Conway moonshine. These results motivate a number of interesting questions. We discuss a few of them here.

- Apart from classifying the symmetry groups of $K 3$ NLSMs as abstract groups, it is also important to know what their actions are on the (BPS) spectrum. In particular, the twining genus can differ for two K3 NLSM symmetries with the same embedding into $\mathrm{Co}_{0}[17,22,54]$. This motivated us to classify the distinct conjugacy classes in $O^{+}\left(\Gamma^{4,20}\right)$ and $O\left(\Gamma^{4,20}\right)$ for a given four-plane preserving Frame shape.

Given this consideration and given our Conjectures 5 and 6 relating twining genera and moonshine functions, an important natural question is the following: Given a particular K3 NLSM, how do we understand which case(s) of umbral moonshine govern its symmetries?

- In this paper, we extend the classification of symmetry groups to singular points in the moduli space of $K 3$ NLSMs. These singular points correspond to perfectly well-defined string compactifications where the physics in the six-dimensional noncompact spacetime involves enhanced non-abelian gauge symmetries. It will be interesting to study the BPS-counting functions arising in these compactifications.

Moreover, as these points are T-dual to type IIB compactifications on K3 in the presence of an NS5-brane [91], it would be interesting to explore the symmetries of these special points from this spacetime point of view. Furthermore, it may also be interesting to classify the symmetry groups in more general fivebrane spacetimes, such as those studied in $[61,62]$ in connection with umbral moonshine.

- More generally, one can try to classify the discrete symmetry groups which arise in other supersymmetric string compactifications, in varying dimensions and with differing numbers of supersymmetries. For example, one case of particular interest is the symmetries of theories preserving only eight supercharges. One difficulty in studying such theories is the global form of the moduli space is often not known, so one does not have the power of lattice embedding theorems used to study theories with 16 supercharges. However, it may be possible to get partial results in certain examples. The connection between sporadic groups, geometry and automorphic forms in theories with eight supercharges has only somewhat been studied (see, e.g., [23,60]), and it would be interesting to explore it further.

- Twining genera of $K 3$ NLSMs can be lifted to twining genera of the $N$ th symmetric product CFT $\operatorname{Sym}^{N}(K 3)$ through a generalization [19] of the formula for the symmetric product elliptic genus of [36]. It can happen that a symmetry which is not a geometric symmetry of any $K 3$ surface can be a geometric symmetry for a hyper- 
Kähler manifold that is deformation equivalent to the $N$-th Hilbert scheme of a $K 3$ surface for $N \geq 2$. The symmetries of such hyper-Kähler manifolds of $K 3^{[N]}$ type were classified in [64] for $N=2$ in terms of their embedding into $\mathrm{Co}_{0}$. This includes Frame shapes corresponding to elements of order 3, 6, 9, 11, 12, 14 and 15 which are not geometric symmetries of any $K 3$ surface. Each of these elements has at least two distinct twining functions associated with it via umbral and Conway moonshine as presented in Table 4. We noticed that for the elements of order 11, 14 and 15, these distinct twining functions lift to the same twined elliptic genus for $\operatorname{Sym}^{N}(K 3)$ for $N=2,3,4$. It would be interesting to understand when this general phenomenon occurs, and more generally the structure of symmetries of string theory on $K 3 \times S^{1}$.

- The compactification of type IIA on $K 3 \times T^{2}$ gives rise to a four-dimensional model with half-maximal supersymmetry (16 supercharges). When the internal NLSM has a symmetry $g$, one can construct a new four-dimensional model (CHL model) with the same number of supersymmetries $[10-12,83]$. The CHL model is defined as the orbifold of type IIA on $\mathrm{K} 3 \times T^{2}$ by a fixed-point-free symmetry acting as $g$ on the K3 sigma model and, simultaneously, by a shift along a circle $S^{1}$ in the $T^{2}$. The twining genus $\mathcal{Z}_{g}$ is directly related to the generating function $1 / \Phi_{g}$ of the degeneracies of $1 / 4$ BPS dyons in this CHL model $[20,29-32,35,36,67,84]$. Up to dualities, the CHL model only depends on the Frame shape of $g$ [80]. This is apparently puzzling for those Frame shapes that correspond to multiple $O^{+}\left(\Gamma^{4,20}\right)$-classes and therefore to multiple twining genera $\mathcal{Z}_{g}$ : In these cases, there are different candidates $1 / \Phi_{g}$ for the $1 / 4$ BPS-counting function, one for each distinct twining genus $\mathcal{Z}_{g}$. Since $O^{+}\left(\Gamma^{4,20}\right)$ is part of the T-duality group of the four-dimensional model, a natural interpretation of this phenomenon is that the different $1 / \Phi_{g}$ functions count $1 / 4$ BPS dyons related to different T-duality orbits of charges in the same CHL model. In view of this interpretation, it would be interesting to understand the precise correspondence between $\mathrm{O}^{+}\left(\Gamma^{4,20}\right)$-classes and $\mathrm{T}$-duality orbits of charges.

- One piece of supporting evidence for our conjectures concerns twining genera with complex multiplier systems. However, so far we have not been able to directly obtain these proposed twining genera from $K 3$ NLSMs. Nevertheless, we argue that this is unsurprising and does not constitute discouraging counter-evidence for our conjectures for the following reason. Recall that the argument in Sect. 3.2 indicates that these functions must arise from a symmetry acting differently on left- and right-movers. Then, our Conjecture 5, together with the observation that such twining functions always arise from multiple instances of umbral and Conway moonshine (see Sect. 4.5, 2nd bullet point), predicts that these theories correspond to lattices embeddable into multiple Niemeier lattices. This precludes most of the exactly solvable models that have been studied so far, in particular all torus orbifolds and some Gepner models, since these always contain a quantum symmetry which can only arise from a Leech embedding. So far most of the NLSM analysis has focussed on these exactly solvable models, and this explains why we have not observed these proposed twining genera yet.

On the other hand, a number of the proposed twining genera with complex multipliers (as well as many with real multipliers) were found by twining certain LG orbifold theories [17]. These include functions arising from symmetries of order 3, 4, 6 and 8 and with Frame shapes $3^{8}, 4^{6}, 6^{4}$ and $4^{2} 8^{2}$-the four Frame shapes which both 
preserve a four-plane in $\mathrm{Co}_{0}$ and correspond to twining genera with complex multiplier. In order to obtain these twining genera, one has to consider symmetries which act asymmetrically on the left- and right-moving fermions in the chiral multiplets, such that the UV Lagrangian, the right-moving $\mathcal{N}=2$ algebra and the four charged Ramond ground states are preserved. In general, however, the left-moving $\mathcal{N}=2$ algebra is not preserved. Though $H_{L}$ and $J_{L}$ must remain invariant for the twining genus to be well defined, $G_{-}$and $\bar{G}_{-}$are transformed under these symmetries, such that the symmetry maps the left-moving $\mathcal{N}=2$ to a different but isomorphic copy. See [17] for more details. It is important to note that though these symmetries do not preserve the full UV supersymmetry algebra, it does not preclude the possibility that they preserve a copy of the IR $\mathcal{N}=(4,4)$ SCA. After all, there is only an $\mathcal{N}=(2,2)$ supersymmetry algebra apparent in the UV, and only after a non-trivial RG flow involving a complicated renormalization of the Kähler potential does the symmetry get enhanced to $\mathcal{N}=(4,4)$ at the conformal point. A clarification of the IR aspects of these UV symmetries would be helpful in unravelling the nature of these left-right asymmetric symmetries.

- While our Conjecture 6 states that all umbral and Conway moonshine functions corresponding to four-plane preserving group elements play a role in the twining genera of $K 3 \mathrm{NLSMs}$, the physical relevance of the umbral (including Mathieu) moonshine functions corresponding to group elements preserving only a two-plane remains unclear. We highlight a number of approaches to this problem here.

One possible approach to the problem is to find a way to combine symmetries realized at different points in moduli space and in this way generate a larger group which also contains two-plane preserving elements. This approach is motivated by the fact that the elliptic genus receives only contributions from BPS states and is invariant across the moduli space. This possibility was first raised as a question "Is it possible that these automorphism groups at isolated points in the moduli space of $K 3$ surface are enhanced to $M_{24}$ over the whole of moduli space when we consider the elliptic genus?" in [46]. Concrete steps toward realizing this idea in the context of Kummer surfaces were taken in $[17,87,88]$. See also [50] for recent progress in the direction.

A second approach is to consider string compactifications where larger groups are realized at given points in moduli space as symmetry groups of the full theory (and not just the BPS sector). For theories with 16 supercharges, this is only possible for compactifications with less than six non-compact dimensions. For example, it was shown that there are points in the moduli space of string theory compactifications to three dimensions which admit the Niemeier groups as discrete symmetry groups [68]. In the type IIA frame, these are given by compactifications on $K 3 \times T^{3}$. The action of these symmetry groups on the 1/2-BPS states of the theory has been analyzed [68], and it would be interesting to understand the action on the 1/4-BPS states.

A third approach stems from the vertex operator algebra (VOA) perspective. In [14], a close variant of the Conway module is shown to exhibit an action of a variety of two-plane preserving subgroups of $\mathrm{Co}_{0}$, including $M_{23}$, and yields as twining genera 
a set of weak Jacobi forms of weight zero and index two. ${ }^{11}$ In addition, the mock modular forms which display $M_{23}$ representations appear to be very closely related to the mock modular forms which play a role in $M_{24}$ moonshine. However, the physical relevance of this module is still unclear. A better understanding of the connection between the Conway module and K3 NLSMs could help explain Mathieu and umbral moonshine.

Finally, yet another approach is to consider compactifications preserving less supersymmetry $[23,60]$. It is not unlikely that the ultimate explanation of umbral moonshine will require a combination of the above approaches.

\begin{abstract}
Author details
${ }^{1}$ Korteweg-de Vries Institute for Mathematics, Amsterdam, The Netherlands, ${ }^{2}$ Institute of Physics, University of Amsterdam, Amsterdam, The Netherlands, ${ }^{3}$ Center for the Fundamental Laws of Nature, Harvard University, Cambridge, MA 02138, USA, ํㅗipartimento di Fisica e Astronomia 'Galileo Galilei' e INFN sez. di Padova, Via Marzolo 8, 35131 Padova, Italy, ${ }^{5}$ Stanford Institute for Theoretical Physics, Department of Physics, Stanford University, Stanford, CA 94305, USA, ${ }^{6}$ Theory Group, SLAC, Menlo Park, CA 94309, USA.

\section{Acknowlegements}

We thank John Duncan, Francesca Ferrari, Matthias Gaberdiel, Gerald Höhn, Shamit Kachru and Natalie Paquette for discussions about related subjects. The work of M. C. is supported by ERC starting Grant H2020 ERC StG 2014. S. M. H. is supported by a Harvard University Golub Fellowship in the Physical Sciences and DOE Grant DE-SC0007870. R. V. is supported by a grant from "Programma per giovani ricercatori Rita Levi Montalcini." M. Z. is supported by the Mellam Family Fellowship at the Stanford Institute for Theoretical Physics.
\end{abstract}

\title{
A Some basic facts about lattices
}

In this appendix, we collect some useful facts about lattices (see $[26,76]$ for more details). Let $L$ be an even lattice with non-degenerate quadratic form $Q: L \rightarrow 2 \mathbb{Z}$ of signature $(n, m)$ and rank $d=n+m$. Let $L^{*}$ be the dual, so that naturally $L \subseteq L^{*}, L^{*} \subset L \otimes_{\mathbb{Z}} \mathbb{Q}$. The quadratic form $Q$ extends to $Q: L^{*} \rightarrow \mathbb{Q}$ by linearity. The discriminant group of $L$

$$
A_{L}:=L^{*} / L
$$

is a finite abelian group, with a discriminant form

$$
q_{L}: A_{L} \rightarrow \mathbb{Q} / 2 \mathbb{Z}
$$

induced by $Q: L^{*} \rightarrow \mathbb{Q}$. The discriminant group has order $\operatorname{det} B_{i j}$ where $B_{i j}$ is the Gram matrix for the quadratic form $Q$ with respect to some basis $v_{1}, \ldots, v_{d}$ of $L$.

We denote by $O(L)$ the group of automorphisms of $L$ (which is the same as the group $O\left(L^{*}\right)$ of automorphisms of the dual $\left.L^{*}\right)$. Similarly, we define $O\left(q_{L}\right)$ as the group of automorphisms of the discriminant group $A_{L}$ that preserves the quadratic form $q_{L}$

$$
O\left(q_{L}\right):=\left\{g \in \operatorname{Aut}\left(A_{L}\right) \mid q_{L}(g(x))=q_{L}(x) \text { for all } x \in A_{L}\right\} .
$$

There is an obvious map

$$
O(L) \rightarrow O\left(q_{L}\right)
$$

which, in general, is neither injective nor surjective.

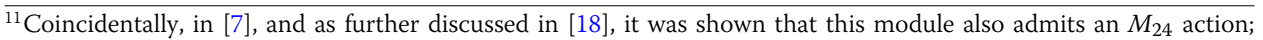
although the twining genera are no longer weak Jacobi forms, the representations are less closely related to those of Mathieu moonshine.
} 
We recall that two even lattices $L, L^{\prime}$ are in the same genus if and only if they have the same signature and the discriminant forms are isomorphic, i.e., there is an isomorphism $\gamma: A_{L} \stackrel{\cong}{\cong} A_{L^{\prime}}$ such that $q_{L^{\prime}} \circ \gamma=q_{L}$ (that the signature and the quadratic form determine the genus is proved in Corollary 1.9.4 in [76]; the converse is trivial; see, for example, Theorem VI.6.1 in [70]). See [26] for the definition of the genus of quadratic forms.

When two discriminant groups $A, A^{\prime}$ with discriminant forms $q, q^{\prime}$ are isomorphic $\gamma$ : $A \stackrel{\cong}{\longrightarrow} A^{\prime}$ and the isomorphism is compatible with the quadratic forms, i.e. $q=q^{\prime} \circ \gamma$, then we simply say that the discriminant forms are isomorphic and use the notation

$$
q \cong q^{\prime}
$$

Let $L$ be an even unimodular lattice and $M$ a primitive sublattice. Recall that $M \subset L$ is said to be a primitive sublattice if the following three equivalent statements are true: 1 . $L / M$ has no torsion; 2. $(M \otimes \mathbb{Q}) \cap L=M$; 3. if $v \in L$ and $n v \in M$ for some $n \in \mathbb{Z}$, then $v \in M$. Let $N$ be the orthogonal complement of $M$ (then, $N$ is automatically primitive), so that

$$
N \oplus M \subseteq L \subseteq N^{*} \oplus M^{*}
$$

Then, there is an isomorphism $\gamma: A_{M} \rightarrow A_{N}$ of discriminant groups, such that

$$
q_{N} \circ \gamma=-q_{M}
$$

and satisfying

$$
L=\left\{(v, w) \in M^{*} \oplus N^{*} \mid \gamma(\bar{v})=\bar{w}\right\},
$$

where $\bar{v} \in A_{M}$ (resp., $\bar{w} \in A_{N}$ ) is the class with representative $v \in M^{*}$ (resp., $w \in N^{*}$ ). Vice versa, given two even lattices $N, M$ with an isomorphism $\gamma: A_{M} \rightarrow A_{N}$ satisfying (A.7), then the lattice $L$ defined by (A.8) is an even unimodular lattice, such that $N, M$ are two mutually orthogonal primitive sublattices of $L$.

\section{B Proofs of results in Sect. 2}

\section{B.1 Proof of Theorem 1}

Recall that, given an even lattice $T$, the discriminant group is the finite abelian group $A_{T}=T^{*} / T$. The quadratic form on $T$ induces a quadratic form $q_{T}: A_{T} \rightarrow \mathbb{Q} / 2 \mathbb{Z}$ on the discriminant group, called the discriminant (quadratic) form.

Theorem 1.12.4 of [76] gives sufficient conditions for the existence of a primitive embedding of an even lattice $T$ of signature $\left(t^{+}, t^{-}\right)$into some even unimodular lattice $L$ of signature $\left(l^{+}, l^{-}\right)$, with $l^{+}-l^{-} \equiv 0 \bmod 8$ :

$$
\left\{\begin{array}{l}
l^{+} \geq t^{+}, l^{-} \geq t^{-} \\
t^{+}+t^{-} \leq \frac{1}{2}\left(l^{+}+l^{-}\right)
\end{array} \quad \Rightarrow \quad \text { exists primitive } T \hookrightarrow L .\right.
$$

Alternatively, a necessary and sufficient condition for such an embedding is the existence of a lattice $K$ of signature $\left(l^{+}-t^{+}, l^{-}-t^{-}\right)$such that

$$
q_{K} \cong-q_{T}
$$


where $q_{K}$ and $q_{T}$ are the discriminant forms of $K$ and $T$ (see [76], Theorem 1.12.2). More precisely, when (B.2) is satisfied, one can construct an even unimodular lattice $L$ such that

$$
K \oplus T \subseteq L \subseteq K^{*} \oplus T^{*}
$$

and such that the embeddings $T \hookrightarrow L$ and $K \hookrightarrow L$ are primitive [76]. Conversely, if $T$ is a primitive sublattice of an even unimodular lattice and $K$ its orthogonal complement, then (B.2) is satisfied.

Since $\Gamma^{G}$ has signature $(4, d)$, by (B.1) it can be primitively embedded into an even unimodular lattice $\Gamma^{8+d, d}$ of signature $(8+d, d)$. Let $S$ be its orthogonal complement in $\Gamma^{8+d, d}$ and $S(-1)$ the lattice obtained by flipping the sign of the quadratic form of $S$. Then, $S(-1)$ has signature $(0,4+d)$ and, using (B.2) repeatedly, we obtain

$$
q_{S(-1)}=-q_{S} \cong q_{\Gamma^{G}} \cong-q_{\Gamma_{G}} .
$$

Thus, there exists an even unimodular lattice $N$ of signature $(0,24)$ such that

$$
\Gamma_{G} \oplus S(-1) \subseteq N \subseteq \Gamma_{G}^{*} \oplus S^{*}(-1)
$$

and such that the embedding $\Gamma_{G} \hookrightarrow N$ is primitive. For the proof of the other claims, see appendix B in [54].

\section{B.2 Proof of Theorem 2}

The proof is completely analogous to the one of Theorem 1 . Since $N^{G}$ has signature $(0,4+d)$, by (B.1) it can be primitively embedded into an even unimodular lattice $\Gamma^{d, 8+d}$ of signature $(d, 8+d)$. Let $S$ be its orthogonal complement in $\Gamma^{d, 8+d}$ and $S(-1)$ the lattice obtained by flipping the sign of the quadratic form of $S$. Then, $S(-1)$ has signature $(4, d)$ and, using (B.2) repeatedly, we obtain

$$
q_{S(-1)}=-q_{S} \cong q_{N^{G}} \cong-q_{N_{G}} .
$$

Thus, since there is a unique (up to isomorphism) even unimodular lattice of signature $(4,20)$, we have

$$
N_{G} \oplus S \subseteq \Gamma^{4,20} \subseteq N_{G}^{*} \oplus S^{*}
$$

and the embedding $N_{G} \hookrightarrow \Gamma^{4,20}$ is primitive. For the proof of the other claims, see appendix B in [54].

\section{B.3 Proof of Proposition 3}

Recall that a Weyl chamber $\mathcal{W} \subset N \otimes \mathbb{R}$ is the closure of any of the connected components in

$$
N \otimes \mathbb{R} \backslash\left(\bigcup_{r \in N, r^{2}=-2} r^{\perp}\right),
$$


the complement of the hyperplanes orthogonal to the roots. The Weyl group $W_{N}$ acts by permutations on the set of Weyl chambers; in particular, for any non-trivial $w \in W_{N}$ and Weyl chamber $\mathcal{W}$, the interior $\mathcal{W}^{o}$ and its image $w\left(\mathcal{W}^{o}\right)$ have no intersection

$$
W^{o} \cap w\left(W^{o}\right)=\emptyset .
$$

Suppose that $\hat{G} \cap W_{N}$ contains some non-trivial element $w$. Since $w$ fixes $N^{\hat{G}}$ pointwise, it follows that the sublattice $N^{\hat{G}}$ cannot contain any vector in the interior of a Weyl chamber. Therefore,

$$
N^{\hat{G}} \subset \bigcup_{r \in N, r^{2}=-2} r^{\perp} .
$$

Since $N^{\hat{G}} \otimes \mathbb{R}$ is convex, it must be actually contained in some hyperplane $r^{\perp}$, for some root $r \in N$, which implies that $r \in N_{\hat{G}} \cong \Gamma_{G}$. Vice versa, if $N_{\hat{G}} \cong \Gamma_{G}$ contains a root $r$, then the corresponding reflection $w_{r} \in W_{N}$ fixes $N^{\hat{G}}$ and therefore is in G. For the second point, notice that the natural projection $O(N) \rightarrow O(N) / W_{N}$ defines a homomorphism from $\hat{G}$ into $G_{N}=O(N) / W_{N}$. As we just proved, if $\Gamma_{G}$ has no roots, then $\hat{G}$ has trivial intersection with $W_{N}$, so that this homomorphism is injective. We hence conclude that $\hat{G}$ is isomorphic to a subgroup of $G_{N}$.

\section{Modular groups and multipliers}

In this appendix, we present the arguments we employed in Sect. 3 to determine the modular properties, in particular the multipliers, of the twining genera. The twining genera $\mathcal{Z}_{g}$ are weak Jacobi forms of weight 0 and index 1 for some subgroup $\mathfrak{G}_{g} \subseteq S L_{2}(\mathbb{Z})$, possibly with a multiplier (group homomorphism) $\psi: \mathfrak{G}_{g} \rightarrow \mathbb{C}^{*}$.

In this appendix, we describe in some more detail the groups $\mathfrak{G}_{g}$ and the multipliers $\psi$. Consider an order- $N$ symmetry $g$. Recall that the modular transformation $\left(\begin{array}{ll}a & b \\ c & d\end{array}\right) \in S L_{2}(\mathbb{Z})$ transforms the twining genus $\mathcal{Z}_{g}$ into a "twisted-twining" genus $\mathcal{Z}_{g^{c}, g^{d}}$, the trace of $g^{d}$ over the $g^{c}$ - twisted sector (see, e.g., [58], section 8.3; we use the convention that a state $|v\rangle$ in the $g$-twisted sector satisfies $\left.g|v\rangle=e^{2 \pi i\left(\bar{L}_{0}-L_{0}\right)}|v\rangle\right)$. As a result, the subgroup $\mathfrak{G}_{g} \subseteq S L_{2}(\mathbb{Z})$ corresponding to transformations fixing $\mathcal{Z}_{g}$ (possibly up to a multiplier) is given by

$$
\mathfrak{G}_{g}:=\left\{\left(\begin{array}{ll}
a & b \\
c & d
\end{array}\right) \in \Gamma_{0}(N) \mid \exists h \in O^{+}\left(\Gamma^{4,20}\right) \text { s.t. } g^{d}=h g h^{-1} \text { or } g^{d}=h g^{-1} h^{-1}\right\},
$$

and is in particular always contained in

$$
\Gamma_{0}(N)=\left\{\left(\begin{array}{ll}
a & b \\
c & d
\end{array}\right) \in S L_{2}(\mathbb{Z}) \mid c=0 \bmod N\right\} .
$$

The group $\mathfrak{G}_{g}$ for each four-plane preserving Frame shape is given in "Appendix D.2."

The order $n$ of the multiplier $\psi$ of $\mathcal{Z}_{g}$ can be determined from the Frame shape of $g$ under some physically motivated assumptions about its form, which we will discuss below. These results, derived using these assumptions, will be used in appendix D to put a lower bound on the number of $O^{+}\left(\Gamma^{4,20}\right)$ classes for the Frame shapes $3^{8}$ and $4^{6}$.

The assumptions are as follows. In general, for (holomorphic) orbifold CFTs, the multiplier is believed to be completely specified in terms of an element of the third cohomology group $H^{3}\left(\mathbb{Z}_{N}, U(1)\right) \cong \mathbb{Z}_{N}$, which determines the modular tensor category of modules over the $g$-invariant subalgebra $[33,34,81]$. We will assume that this is true for $K 3$ NLSMs 
and their orbifolds. Furthermore, we will assume that the triviality of the multiplier $\psi$ is the only condition for the $g$-orbifold to be a consistent CFT. See also recent results on generalized umbral moonshine $[13,55]$ for more detail about the relations between multipliers and third cohomology. Under these assumptions, the order $n$ of the multiplier of $\mathcal{Z}_{g}$ is always a divisor of $N$. Second, for any divisor $d \mid n$, the element $g^{d}$ has multiplier of order $n / d$. In particular, $g^{n}$ is the smallest power of $g$ with trivial multiplier. As a result, if the $g^{K}$-orbifold of the NLSM is again a consistent CFT, necessarily an $\mathcal{N}=(4,4)$ superconformal field theory at central charge 6 , our assumption then dictates that $n$ divides $K$.

The above considerations, together with an analysis of the Witten index of the orbifold theory which we now explain, lead to a derivation of lower and upper bounds on $n$ for a given $g$. There is a general formula for the Witten index of an orbifold by a cyclic group $\langle g\rangle$. In terms of the Frame shape $\pi_{g}=\prod_{\ell \mid N} \ell^{k_{\ell}}$ of $g$, this is given by

$$
\sum_{\ell \mid N} \frac{N}{\ell} k_{\ell}
$$

(see, e.g., [51] or [80] for a proof). On the other hand, the only possible Witten indices of an $\mathcal{N}=(4,4)$ SCFT with central charge 6 and integer $U(1)$ charges are 0 or 24 [75]. Therefore, if for some $g$ the putative Witten index (C.2) of the $g$ orbifold is different from 0 or 24, then the orbifold is necessarily inconsistent, and the corresponding multiplier must be non-trivial $(n>1)$. An upper bound on $n$ can be obtained by noticing that, by (4.21), the multiplier must be trivial $(n=1)$ whenever $\operatorname{Tr}_{V_{24}}(g) \neq 0$. By studying which powers of $g$ have a potentially non-trivial multiplier (i.e. $\operatorname{Tr}_{V_{24}}\left(g^{n}\right)=0$ ) and which powers give a potentially consistent orbifold (i.e., (C.2) is either 0 or 24), one derives lower and upper bounds on the order of the multiplier of $g$, which are sufficient to determine $n$ in all cases. For almost all the four-plane preserving Frame shapes $\prod_{\ell \mid N} \ell^{k_{\ell}}$, the order $n$ is the value of the smallest $\ell$ for which $k_{\ell} \neq 0$. The only exception is $2^{-4} 4^{8}$, which has trivial multiplier, as follows from the fact that it is the square of an element of Frame shape $1{ }^{4} 2^{-2} 4^{-2} 8^{4}$, which has nonzero trace and therefore $n=1$.

A case-by-case analysis for all the four-plane preserving Frame shapes shows that the possible orders of a non-trivial multiplier are $n \in\{2,3,4,6\}$. For each such $n$, the possible forms of the corresponding multiplier $\psi$ are in one-to-one correspondence with the classes of order $n$ in the third cohomology group $H^{3}\left(\mathbb{Z}_{N}, U(1)\right) \cong \mathbb{Z}_{N}$. In particular, for $n=2$, there is only one possible multiplier $\psi$, while for each $n=3,4$ or 6 there are two possible multipliers $\psi$ and $\bar{\psi}$ that are complex conjugate to each other. By the arguments in Sect. 3.2, when $n>2$, both $\psi$ and $\bar{\psi}$ must appear as the multipliers associated with some physical twining genera.

\section{Some classification results}

\section{D.1 Classification of $O^{+}\left(\Gamma^{4,20}\right)$ conjugacy classes}

Consider a four-plane preserving element of $\mathrm{O}^{+}\left(\Gamma^{4,20}\right)$. Its eigenvalues in the 24dimensional defining representation can be encoded in a Frame shape. See Sect. 3. Given such a Frame shape, in this appendix we compute the number of compatible $O^{+}\left(\Gamma^{4,20}\right)$ conjugacy classes. More specifically, we first discuss a theorem (Theorem 7) which we 
will employ to determine the number of $O^{+}\left(\Gamma^{4,20}\right)$ conjugacy classes with a given Frame shape, as recorded in Table 4.

Let $\Lambda$ be the (negative definite) Leech lattice, $\hat{g} \in O(\Lambda) \cong C o_{0}$ be an automorphism with Frame shape $\pi_{g}$ and fixing a sublattice $\Lambda^{\hat{g}}$ of rank $4+d, d \geq 0$ and let $\Lambda_{\hat{g}}$ (the co-invariant lattice) be the orthogonal complement of $\Lambda^{\hat{g}}$ in $\Lambda$. We denote by $O\left(\Lambda_{\hat{g}}\right)$ the group of automorphisms of $\Lambda_{\hat{g}}$ and by

$$
C_{O\left(\Lambda_{\hat{g}}\right)}(\hat{g}):=\left\{\hat{h} \in O\left(\Lambda_{\hat{g}}\right) \mid \hat{g} \hat{h}=\hat{h} \hat{g}\right\}
$$

the centralizer of $\hat{g}$ in this group.

Given a primitive embedding

$$
i: \Lambda_{\hat{g}} \hookrightarrow \Gamma^{4,20},
$$

denote the image of $i$ and its orthogonal complement by

$$
\Gamma_{g}:=i\left(\Lambda_{\hat{g}}\right) \quad \Gamma^{g}:=\Gamma^{4,20} \cap\left(\Gamma_{g}\right)^{\perp} .
$$

The embedding induces an automorphism $g \in O^{+}\left(\Gamma^{4,20}\right)$ which acts as $\hat{g}$ on the image $i\left(\Lambda_{\hat{g}}\right)$ and trivially on its orthogonal complement $i\left(\Lambda_{\hat{g}}\right)^{\perp} \cap \Gamma^{4,20}$. Namely, we have

$$
g \circ i=\left.i \circ \hat{g} \quad g\right|_{\Gamma^{g}}=\left.\mathrm{id}\right|_{\Gamma^{g}} .
$$

Note that given $\hat{g}$ and $i$, the above fixes $g$ completely. Moreover, as the notation suggests, $\Gamma^{g}$ is the sublattice of vectors in $\Gamma^{4,20}$ fixed by $g$.

The lattice $\Gamma^{g}$ has signature $(4, d)$, and by (A.7), its discriminant form $q_{\Gamma^{g}}$ must be the opposite of $q_{\Lambda_{\hat{g}}}$ :

$$
\operatorname{sign}\left(\Gamma^{g}\right)=(4, d), \quad q_{\Gamma^{g}} \cong-q_{\Lambda_{\hat{g}}} .
$$

As a consequence, the genus of $\Gamma^{g}$ is determined uniquely in terms of $\Lambda_{\hat{g}}$, independently of the embedding $i$. We denote by $\operatorname{cl}(\hat{g})$ the set of isomorphism classes of lattices in this genus.

Conversely, every $g \in O^{+}\left(\Gamma^{4,20}\right)$ fixing a sublattice of signature $(4, d)$ with $d \geq 0$ can be obtained in this way: namely, (D.4) for some $\hat{g} \in \mathrm{Co}_{0}$ and some primitive embedding $i$. As discussed in Sect. 3.1, two such automorphisms $g_{1}$ and $g_{2}$ have the same Frame shape if and only if they can be induced by the same $\hat{g} \in \mathrm{Co}_{0}$, possibly with different embeddings $i_{1}$, and $i_{2}$. In particular, if $g_{1}$ and $g_{2}$ are conjugated within $O^{+}\left(\Gamma^{4,20}\right)$, then they are necessarily induced by the same $\hat{g}$. The converse statement is, however, not true in general: It can happen that two $g_{1}$ and $g_{2}$ are induced from the same $\hat{g}$ (i.e., they have the same Frame shape), but they are not conjugated in $O^{+}\left(\Gamma^{4,20}\right)$. The following theorem 7 will enable us to determine the number of $\mathrm{O}^{+}\left(\Gamma^{4,20}\right)$ conjugacy classes arising from a given Frame shape, for all four-plane fixing elements of $\mathrm{Co}_{0}$ with the exception of the Frame shape $1^{-4} 2^{5} 3^{4} 6^{1}$ which we will discuss at the end of this appendix.

To understand the theorem, recall that a (positive) sign structure for a lattice $L$ is a choice of orientation of a maximal positive definite subspace in $L \otimes_{\mathbb{Z}} \mathbb{R} .^{12}$ We denote by

${ }^{12}$ Similarly, a negative sign structure is given by a choice of orientation of a maximal negative-definite subspace in $L \otimes_{\mathbb{Z}} \mathbb{R}$. In the following, we will only consider positive sign structures and simply refer to them as sign structures. 
$\mathrm{Cl}^{+}(\hat{g})$ the set of classes of lattices with sign structure in the genus (D.5). In other words, two lattices $L_{1}, L_{2}$ are equivalent if there is an isomorphism $L_{1} \rightarrow L_{2}$ that preserves the orientation of maximal positive-definite subspaces.

Theorem 7 Let $^{+} \mathrm{Cl}^{+}(\hat{g})$ be the set of equivalence classes of lattices with a sign structure in the genus determined by (D.5). Then, the number of $\mathrm{O}^{+}\left(\Gamma^{4,20}\right)$ conjugacy classes with Frame shape $\pi_{g}$ is given by

$$
\sum_{K \in c l^{+}(\hat{g})}\left|\bar{C}_{O\left(\Lambda_{\hat{g}}\right)}(\hat{g}) \backslash O\left(q_{\Lambda_{\hat{g}}}\right) / \gamma_{K} *\left(\overline{O^{+}}(K)\right)\right|,
$$

where the sum is over a set of representatives for the isomorphism classes in $\mathrm{Cl}^{+}(\hat{g})$, $\overline{\mathrm{O}^{+}}(K)$ (respectively, $\left.\bar{C}_{O\left(\Lambda_{\hat{g}}\right)}(\hat{g})\right)$ is the image of the natural map $O^{+}(K) \rightarrow O\left(q_{K}\right)$ (resp. $\left.C_{O\left(\Lambda_{\hat{g}}\right.}(\hat{g}) \rightarrow O\left(q_{\Lambda_{\hat{g}}}\right)\right)$. Furthermore, for each $K, \gamma_{K}: A_{K} \stackrel{\cong}{\cong} A_{\Lambda_{\hat{g}}}$ is an isomorphism of discriminant groups, with $q_{K}=-q_{\Lambda_{\hat{g}}} \circ \gamma_{K}$, and $\gamma_{K}^{*}\left(O\left(q_{K}\right)\right):=\gamma_{K} O\left(q_{K}\right) \gamma_{K}^{-1}=O\left(q_{\Lambda_{\hat{g}}}\right)$ the induced identification of the orthogonal groups. The number of classes does not depend on the choice of these isomorphisms.

The first step in proving this theorem is to determine when two different embeddings $i_{1}$ and $i_{2}$ give rise to $g_{1}$ and $g_{2}$ that are conjugated in $O^{+}\left(\Gamma^{4,20}\right)$.

Lemma 8 Let $i_{1}$ and $i_{2}$ be primitive embeddings of $\Lambda_{\hat{g}}$ into $\Gamma^{4,20}$ and $g_{1}, g_{2} \in O^{+}\left(\Gamma^{4,20}\right)$ be the automorphisms determined by (D.4). Then, $g_{1}$ and $g_{2}$ are conjugated in $\mathrm{O}^{+}\left(\Gamma^{4,20}\right)$ if and only if there exist $s \in C_{O\left(\Lambda_{\hat{g}}\right)}(\hat{g})$ and $h \in O^{+}\left(\Gamma^{4,20}\right)$, such that

$$
h \circ i_{1}=i_{2} \circ s,
$$

and in this case $g_{2}=h g_{1} h^{-1}$.

Proof Suppose there are $h$ and $s$ such that $h \circ i_{1}=i_{2} \circ s$. Then, $h$ induces an isomorphism of the sublattices $i_{1}\left(\Lambda_{\hat{g}}\right)$ and $i_{2}\left(\Lambda_{\hat{g}}\right)$ and the orthogonal complements $h\left(i_{1}\left(\Lambda_{\hat{g}}\right)^{\perp}\right)=i_{2}\left(\Lambda_{\hat{g}}\right)^{\perp}$. This implies that

$$
\left.\left(h g_{1} h^{-1}\right)\right|_{i_{2}\left(\Lambda_{\hat{g}}\right)^{\perp}}=\left.\mathrm{id}\right|_{i_{2}\left(\Lambda_{\hat{g}}\right)^{\perp}} .
$$

Furthermore, the condition $g_{1} i_{1}=i_{1} \hat{g}$ and the analogue for $g_{2}, i_{2}$ (we drop $\circ$ from now on) imply

$$
h g_{1} i_{1}=h i_{1} \hat{g}=i_{2} s \hat{g}=i_{2} \hat{g} s=g_{2} i_{2} s=g_{2} h i_{1} .
$$

Using again $h\left(i_{1}\left(\Lambda_{\hat{g}}\right)\right)=i_{2}\left(\Lambda_{\hat{g}}\right)$, it follows that

$$
\left.\left(h g_{1} h^{-1}\right)\right|_{i_{2}\left(\Lambda_{\hat{g}}\right)}=\left.g_{2}\right|_{i_{2}\left(\Lambda_{\hat{g}}\right)} .
$$

As a result, since $h g_{1} h^{-1}$ coincides with $g_{2}$ both on $i_{2}\left(\Lambda_{\hat{g}}\right)$ and on its orthogonal complement, they must be the same.

In the other direction, suppose that $g_{2}=h g_{1} h^{-1}$ for some $h \in O^{+}\left(\Gamma^{4,20}\right)$. It is easy to see that $g_{2}$ acts trivially on $h\left(i_{1}\left(\Lambda_{\hat{g}}\right)^{\perp}\right)$ and hence $h\left(i_{1}\left(\Lambda_{\hat{g}}\right)^{\perp}\right)=i_{2}\left(\Lambda_{\hat{g}}\right)^{\perp}$ and $h i_{1}\left(\Lambda_{\hat{g}}\right)=i_{2}\left(\Lambda_{\hat{g}}\right)$. From this and the identity

$$
h i_{1} \hat{g}=h g_{1} i_{1}=g_{2} h i_{1} .
$$


we get

$$
i_{2}^{-1} h i_{1} \hat{g}=i_{2}^{-1} g_{2} h i_{1}=\hat{g} i_{2}^{-1} h i_{1}
$$

This implies that the automorphism $s:=i_{2}^{-1} h i_{1} \in O\left(\Lambda_{\hat{g}}\right)$ commutes with $\hat{g}$ and, by definition, $h i_{1}=i_{2} s$.

As a result, the $\mathrm{O}^{+}\left(\Gamma^{4,20}\right)$ conjugacy classes corresponding to a given four-plane preserving Frame shape $\pi_{g}$ of $\mathrm{Co}_{0}$ are in one-to-one correspondence with the classes of primitive embeddings $i: \Lambda_{\hat{g}} \hookrightarrow \Gamma^{4,20}$, modulo $O^{+}\left(\Gamma^{4,20}\right) \times C_{O\left(\Lambda_{\hat{g}}\right)}(\hat{g})$. We are now ready to prove Theorem 7 .

Proof For each isomorphism class in $\mathrm{Cl}^{+}(\hat{g})$, we choose once and for all a representative $K$ and an isomorphism $\gamma_{K}: A_{K} \cong A_{\Lambda_{\hat{g}}}, q_{K}=-q_{\Lambda_{\hat{g}}} \circ \gamma_{K}$, and identify $O\left(q_{\Lambda_{\hat{g}}}\right)$ with $\gamma_{K} O\left(q_{K}\right) \gamma_{K}^{-1}$. Note that any other such isomorphism is obtained by composing $\gamma_{K}$ with an element in $O\left(q \Lambda_{\hat{g}}\right)$.

As shown above, the $O^{+}\left(\Gamma^{4,20}\right)$ conjugacy classes with Frame shape $\pi_{g}$ are in oneto-one correspondence with classes of primitive embeddings $i: \Lambda_{\hat{g}} \hookrightarrow \Gamma^{4,20}$, modulo $O^{+}\left(\Gamma^{4,20}\right) \times C_{O\left(\Lambda_{\hat{g}}\right)}(\hat{g})$. We will prove that $O^{+}\left(\Gamma^{4,20}\right)$ classes of embeddings are in oneto-one correspondence with pairs $(K,[t])$, where $K$ runs over our set of representatives of the classes in $c l^{+}(\hat{g})$, and $[t]$ is a double coset in $\bar{C}_{O\left(\Lambda_{\hat{g}}\right)}(\hat{g}) \backslash O\left(q_{\Lambda_{\hat{g}}}\right) / \overline{O^{+}}(K)$. Given such a pair $(K,[t])$, choose an element $t \in O\left(q_{\Lambda_{\hat{g}}}\right)$ in the double coset $[t]$ and consider the lattice

$$
\Gamma_{K, t}:=\left\{(v, w) \in K^{*} \oplus \Lambda_{\hat{g}}^{*} \mid t \circ \gamma_{K}(\bar{v})=\bar{w}\right\}
$$

Since $t \circ \gamma_{K}$ is an isomorphism $q_{K} \rightarrow-q_{\Lambda_{\hat{g}}}, \Gamma_{K, t}$ is an even unimodular lattice of signature $(4,20)$ and there is a sign structure-preserving isomorphism $f: \Gamma_{K, t} \cong \Gamma^{4,20}$. This isomorphism determines a primitive embedding $i: \Lambda_{\hat{g}} \rightarrow \Gamma^{4,20}$ by $i(w)=f((0, w)) \in \Gamma^{4,20}$, which depends on the choice of the isomorphism $f$ only up to composition with $O^{+}\left(\Gamma^{4,20}\right)$. Let $t^{\prime}$ be a different element in the double coset $[t]$, so that $t^{\prime}=\bar{s} t\left(\gamma_{K} \bar{\sigma} \gamma_{K}^{-1}\right)$ for some $s \in C_{O\left(\Lambda_{\hat{g}}\right)}(\hat{g})$ and $\sigma \in O^{+}(K)$. Then, there is an isomorphism $f^{\prime}: \Gamma_{K, t^{\prime}} \rightarrow \Gamma^{4,20}$ given by the composition of the isomorphism $\Gamma_{K, t^{\prime}} \rightarrow \Gamma_{K, t}$ defined by $(v, w) \mapsto\left(\sigma(v), s^{-1}(w)\right)$, followed by $f$. We denote by $i^{\prime}: \Lambda_{\hat{g}} \hookrightarrow \Gamma^{4,20}$ the primitive embedding such that $i^{\prime}(w)=f^{\prime}((0, w))$. Thus, the embeddings $i$ and $i^{\prime}$ are related by $i(w)=i^{\prime}(s(w))$. We conclude that there is a well-defined function mapping pairs $(K,[t])$ to classes of primitive embeddings modulo $O^{+}\left(\Gamma^{4,20}\right) \times C_{O\left(\Lambda_{\hat{g}}\right)}(\hat{g})$.

Let us now consider a primitive embedding $i: \Lambda_{\hat{g}} \hookrightarrow \Gamma^{4,20}$. This embedding determines a description of $\Gamma^{4,20}$ as a sublattice of $\left(\Gamma^{g}\right)^{*} \oplus i\left(\Lambda_{\hat{g}}^{*}\right)$,

$$
\Gamma^{4,20}=\left\{(v, i(w)) \in\left(\Gamma^{g}\right)^{*} \oplus i\left(\Lambda_{\hat{g}}^{*}\right) \mid \gamma_{i}(\bar{v})=\bar{w}\right\}
$$

where, as described before, $\Gamma^{g}:=\left(i\left(\Lambda_{\hat{g}}\right)\right)^{\perp} \cap \Gamma^{4,20}$ and $g \in O^{+}\left(\Gamma^{4,20}\right)$ is given by (D.4). In this formula, $\gamma_{i}$ is some isomorphism $\gamma_{i}: q_{\Gamma} \stackrel{\cong}{\rightrightarrows}-q_{\Lambda_{\hat{g}}}$ of discriminant forms (see (A.8)). Since $\Gamma^{g}$ satisfies (D.5), there exists a sign structure-preserving isomorphism $\Gamma^{g} \cong K$ with one of the representatives of the classes in $\mathrm{Cl}^{+}(\hat{g})$. This isomorphism determines an element $t \in O\left(q_{\Lambda_{\hat{g}}}\right)$ such that $\gamma_{i}=t \gamma_{K}$; elements corresponding to different choices of the isomorphism are related by composition by $\bar{O}^{+}(K)$. Consider two primitive embeddings 
$i_{1}, i_{2}$, and let $g_{1}, g_{2} \in O^{+}\left(\Gamma^{4,20}\right)$ be determined by the embeddings $i_{1}$ and $i_{2}$ via (D.4), so that $\Gamma^{g_{a}}=i_{a}\left(\Lambda_{\hat{g}}\right)^{\perp} \cap \Gamma^{4,20}, a=1,2$. If $i_{1}, i_{2}$ are related by $h i_{1}=i_{2} s^{-1}$, for some $h \in O^{+}\left(\Gamma^{4,20}\right)$ and $s \in C_{O\left(\Lambda_{\hat{g}}\right)}(\hat{g})$, then there is a sign structure-preserving isomorphism $\sigma: \Gamma^{g_{1}} \stackrel{\cong}{\rightarrow} \Gamma^{g_{2}}$ given by the restriction of $h$ to $\Gamma^{g_{1}}$

$$
\sigma(v)=h(v), \quad \forall v \in \Gamma^{g_{1}}
$$

and such that

$$
\bar{s} \gamma_{i_{2}} \bar{\sigma}=\gamma_{i_{1}}
$$

where $\bar{s}$ and $\bar{\sigma}$ are the induced maps on the discriminant forms. Therefore, the elements $t_{1}, t_{2} \in O\left(q_{\Lambda_{\hat{g}}}\right)$ such that $\gamma_{i_{k}}=t_{k} \gamma_{K}$ are related by

$$
t_{1}=\bar{s} t_{2}\left(\gamma_{K} \bar{\sigma} \gamma_{K}^{-1}\right),
$$

so that $t_{1}$ and $t_{2}$ belong to the same double coset in $\bar{C}_{O\left(\Lambda_{\hat{g}}\right)}(\hat{g}) \backslash O\left(q_{\Lambda_{\hat{g}}}\right) / \overline{O^{+}}(K)$. Therefore, there is a well-defined function that maps classes of primitive embeddings into pairs $(K,[t])$, and this function is the inverse of the map defined above.

When the rank of $\Lambda_{\hat{g}}$ is exactly 20, i.e., when the lattices in $\mathrm{Cl}^{+}(\hat{g})$ are positive definite, the groups $O^{+}(K)$ and $C_{O\left(\Lambda_{\hat{g}}\right)}(\hat{g})$ are finite and the number of classes can be computed directly. This paper is accompanied by a text file containing the Magma program we wrote to perform this calculation. In the course of this calculation, we make use of results in [65] classifying sublattices of the Leech lattice fixed by subgroups of $\mathrm{Co}_{0}$.

When the rank of $\Lambda_{\hat{g}}$ is less than 20, a brute force computation is not available, since the groups $O^{+}(K)$ have infinite order. Nevertheless, we can determine lower and upper bounds on the number of $O^{+}\left(\Gamma^{4,20}\right)$ classes. Firstly, we know that, for each Frame shape, there is at least one $O^{+}\left(\Gamma^{4,20}\right)$ class. Miranda and Morrison [71,72] provide a practical algorithm to compute the number of right cosets $\sum_{K \in c l^{+}(g)}\left|O\left(q_{\Lambda_{\hat{g}}}\right) / \overline{O^{+}}(K)\right|$ in the case where $K$ is indefinite with rank at least three. This provides an upper bound on the number of $O^{+}\left(\Gamma^{4,20}\right)$-classes in the case where $\Lambda_{\hat{g}}$ has rank less than 20. This upper bound is almost always sharp-it is equal to one in all cases where the rank of $\Lambda_{\hat{g}}$ is less than 20 , with the exception of the Frame shapes $3^{8}, 4^{6}$, and $1^{-4} 2^{5} 3^{4} 6^{1}$, where there might be at most two classes. Furthermore, when the corresponding twining genus has complex multiplier, the number of $O^{+}\left(\Gamma^{4,20}\right)$ classes must be at least two. Our analysis in "Appendix C" (which is based on some assumptions about modular properties of the twining genera and consistency of the orbifolds) indicates that the twining genera for the Frame shapes $3^{8}$ and $4^{6}$ have complex multiplier of order 3 and 4 , respectively, so there must be exactly two $\mathrm{O}^{+}\left(\Gamma^{4,20}\right)$ classes in these cases. The only undecided case is the Frame shape $1^{-4} 2^{5} 3^{4} 6^{1}$, for which we were not able to determine whether the number of associated $O^{+}\left(\Gamma^{4,20}\right)$ classes is one or two. On the other hand, even if there are two classes, they are necessarily the inverse of each other. This implies that the twining genera are the same. All results are collected in "Appendix D.2."

To summarize:

- For all possible Frame shapes of four-plane preserving elements of $O^{+}\left(\Gamma^{4,20}\right)$, except $3^{8}, 4^{6}$ and $1^{-4} 2^{5} 3^{4} 6^{1}$, the number of $O^{+}\left(\Gamma^{4,20}\right)$ and $O\left(\Gamma^{4,20}\right)$ classes is exactly as shown in the tables in Appendix D.2; 
- for the Frame shapes $3^{8}$ and $4^{6}$, there is a single $O\left(\Gamma^{4,20}\right)$ conjugacy class are at most two $O^{+}\left(\Gamma^{4,20}\right)$ classes; the number of $O^{+}\left(\Gamma^{4,20}\right)$ classes is exactly two if the orders of the multipliers of the corresponding twining genera are as described in "Appendix C";

- for the class $1^{-4} 2^{5} 3^{4} 6^{1}$, there are at most two $O^{+}\left(\Gamma^{4,20}\right)$ conjugacy classes and at most two $O\left(\Gamma^{4,20}\right)$ conjugacy classes.

\section{D.2 Twining genera}

In this subsection, we present the classification of four-plane preserving $\mathrm{O}^{+}\left(\Gamma^{4,20}\right)$ conjugacy classes and our results on the corresponding twining genera. The results are summarized in Table 4.

The first column $\pi_{g}$ contains the 42 possible Frame shapes of four-plane preserving classes of $\mathrm{O}^{+}\left(\Gamma^{4,20}\right)$, which are in one-to-one correspondence with the $42 \mathrm{Co}_{0}$ conjugacy classes of automorphisms of the Leech lattice that fix a sublattice of rank at least 4 [25].

For each Frame shape $\pi_{g}$, the corresponding twining genera are weak Jacobi forms of weight 0 and index 1 for a subgroup $\mathfrak{G}_{g} \subseteq S L_{2}(\mathbb{Z})$, defined in (C.1), with a multiplier $\psi$ of order $n$. In the second column, we list the group $\mathfrak{G}_{g}$ and the order $n$ of the multiplier $\psi$. We use the following notation to describe $\mathfrak{G}_{g}$. If $\kappa$ is a subgroup of $(\mathbb{Z} / N \mathbb{Z})^{\times}$, then we define

$$
\Gamma_{\kappa}(N)=\left\{\left(\begin{array}{ll}
a & b \\
c & d
\end{array}\right) \in S L_{2}(\mathbb{Z}) \mid c \equiv 0(\bmod N), \quad a, d(\bmod N) \in \kappa\right\} .
$$

In this notation, the standard congruence subgroups $\Gamma_{0}(N)$ and $\Gamma_{1}(N)$ correspond to $\Gamma_{\kappa}(N)$ with $\kappa=(\mathbb{Z} / N \mathbb{Z})^{\times}$and $\kappa=\langle 1\rangle$, respectively. Apart from these standard congruence subgroups, we also encounter groups with $\kappa=\langle-1\rangle=\{1,-1\}$. We use the symbol $\Gamma_{\kappa}(N)_{\mid \eta}$ if the twining genus is a Jacobi form for the group $\Gamma_{\kappa}(N)$ with multiplier of order $n>1$. When $n=1$, we simply write $\Gamma_{\kappa}(N)$. Note that, as discussed in appendix $\mathrm{C}$, in general specifying $\mathfrak{G}_{g}$ and $n$ is not sufficient to fix $\psi$ uniquely.

The third and fourth columns report, respectively, the number of $O\left(\Gamma^{4,20}\right)$ and $O^{+}\left(\Gamma^{4,20}\right)$ conjugacy classes of each Frame shape. More precisely, in the third column, we put a symbol $\circ$ for each $O\left(\Gamma^{4,20}\right)$ class. In the fourth column, we put a symbol $\circ$ for each $O^{+}\left(\Gamma^{4,20}\right)$-class that is fixed by world-sheet parity (i.e., it is a class also with respect to the full $O\left(\Gamma^{4,20}\right)$ group) and a symbol $\uparrow$ for each pair of $O^{+}\left(\Gamma^{4,20}\right)$-classes that are exchanged under world-sheet parity (i.e., they merge to form a unique $O\left(\Gamma^{4,20}\right)$ class). We are able to determine the number of such classes for all Frame shapes, except for $1^{-4} 2^{5} 3^{4} 6^{1}$. In this case, there might be either a single class or two classes corresponding to inverse elements $g, g^{-1} \in O^{+}\left(\Gamma^{4,20}\right)$. Notice that whenever $\mathfrak{G}_{g}$ is of the form $\Gamma_{\langle-1\rangle}(N)$, there are always exactly two $O^{+}\left(\Gamma^{4,20}\right)$ classes $[g],\left[g^{\prime}\right]$ (which may or may not be related by world-sheet parity), which are related by a power map, i.e., $\left[g^{\prime}\right]=\left[g^{a}\right]$ for some $a$ coprime to $N$. The corresponding twining genera are distinct, but are related by $\Gamma_{0}(N)$ transformations that are not in $\Gamma_{\langle-1\rangle}(N)$.

The fifth column reports, for each $O^{+}\left(\Gamma^{4,20}\right)$ class, whether the corresponding twining genus $\mathcal{Z}_{g}$ is known in the following sense. A $\checkmark$ denotes a twining function which has been observed in an (IR) K3 NLSM. LG denotes twining functions that have not been observed in an IR K3 NLSM but have been computed in [17] as the twining genus of a UV symmetry in a LG orbifold which flows to a K3 NLSM in the IR, as discussed in Sect. 4.3. 
Table 4 Conjugacy classes, status of the twining genera and the corresponding Niemeier moonshine, for all the four-plane preserving Frame shapes

\begin{tabular}{|c|c|c|c|c|c|}
\hline$\pi_{g}$ & $\left(\mathfrak{G}_{g}\right)_{\mid n}$ & $O\left(\Gamma^{4,20}\right)$ classes & $\mathrm{O}^{+}\left(\Gamma^{4,20}\right)$ classes & $\mathcal{Z}_{g}$ & $\phi_{g}^{N}$ \\
\hline $1^{24}$ & $S L_{2}(\mathbb{Z})$ & o & o & $\checkmark$ & All \\
\hline $1^{8} 2^{8}$ & $\Gamma_{0}(2)$ & $\circ$ & $\circ$ & $\checkmark$ & All except $A_{6}^{4}, A_{12}^{2}, D_{12}^{2}, A_{24}, D_{16} E_{8}, D_{24}$ \\
\hline $1^{-8} 2^{16}$ & $\Gamma_{0}(2)$ & $\circ$ & $\circ$ & $\checkmark$ & $\Lambda$ \\
\hline $2^{12}$ & $\Gamma_{0}(2)_{\mid 2}$ & $\circ$ & $\circ$ & $\checkmark$ & $A_{1}^{24}, A_{2}^{12}, A_{3}^{8}, A_{4}^{6}, D_{4}^{6}, A_{6}^{4}, A_{8,}^{3} D_{6}^{4}, A_{12}^{2}, D_{12}^{2}, A_{24}, \Lambda$ \\
\hline $1^{6} 3^{6}$ & $\Gamma_{0}(3)$ & $\circ$ & $\circ$ & $\checkmark$ & $A_{1}^{24}, A_{2}^{12}, A_{3}^{8}, A_{5}^{4} D_{4}, D_{4}^{6}, A_{6}^{4}, D_{6}^{4}, E_{6}^{4}, \Lambda$ \\
\hline $1^{-3} 3^{9}$ & $\Gamma_{0}(3)$ & $\circ$ & $\circ$ & $\checkmark$ & $\Lambda$ \\
\hline $3^{8}$ & $\Gamma_{0}(3)_{\mid 3}$ & $\circ$ & $\uparrow$ & $\begin{array}{l}L G^{\dagger} \\
x^{\dagger}\end{array}$ & $\begin{array}{l}A_{2}^{12}, D_{4}^{6}, A_{8}^{3}, E_{8}^{3}, \Lambda \\
A_{1}^{24}, A_{4}^{6}, D_{8}^{3}\end{array}$ \\
\hline $1^{4} 2^{2} 4^{4}$ & $\Gamma_{0}(4)$ & $\circ$ & $\circ$ & $\checkmark$ & $A_{1}^{24}, A_{2}^{12}, A_{3}^{8}, A_{4}^{6}, A_{5}^{4} D_{4}, D_{4}^{6}, A_{9}^{2} D_{6}, \Lambda$ \\
\hline $1^{8} 2^{-8} 4^{8}$ & $\Gamma_{0}(4)$ & $\circ$ & 。 & $x$ & $\Lambda$ \\
\hline $1^{-4} 2^{6} 4^{4}$ & $\Gamma_{0}(4)$ & $\circ$ & $\circ$ & $\checkmark$ & $\Lambda$ \\
\hline $2^{-4} 4^{8}$ & $\Gamma_{0}(4)$ & $\circ$ & $\uparrow$ & $\begin{array}{l}\checkmark \\
\checkmark\end{array}$ & $\begin{array}{l}\Lambda_{+} \\
\Lambda_{-}\end{array}$ \\
\hline $2^{4} 4^{4}$ & $\Gamma_{0}(4)_{\mid 2}$ & $\circ$ & ○ & $\checkmark$ & $A_{1}^{24}, A_{2}^{12}, A_{3}^{8}, D_{4}^{6}, A_{7}^{2} D_{5}^{2}, E_{6}^{4}, \Lambda$ \\
\hline $4^{6}$ & $\Gamma_{0}(4)_{\mid 4}$ & $\circ$ & $\uparrow$ & $\begin{array}{l}L G^{\dagger} \\
x^{\dagger}\end{array}$ & $\begin{array}{l}A_{1}^{24}, A_{2}^{12}, A_{6}^{4}, D_{6}^{4} \\
A_{3}^{8}, A_{4}^{6}, A_{12}^{2}, \Lambda\end{array}$ \\
\hline $1^{4} 5^{4}$ & $\Gamma_{0}(5)$ & $\circ$ & $\circ$ & $\checkmark$ & $A_{1}^{24}, A_{2}^{12}, A_{4}^{6}, D_{4}^{6}, \Lambda$ \\
\hline $1^{-1} 5^{5}$ & $\Gamma_{\langle-1\rangle}(5)$ & $\circ$ & $\uparrow$ & $\begin{array}{l}\checkmark \\
\checkmark\end{array}$ & $\begin{array}{l}\Lambda_{+} \\
\Lambda_{-}\end{array}$ \\
\hline $1^{2} 2^{2} 3^{2} 6^{2}$ & $\Gamma_{0}(6)$ & $\circ$ & 。 & $\checkmark$ & $A_{1}^{24}, A_{2}^{12}, A_{3}^{8}, D_{4}^{6}, E_{6}^{4}, \Lambda$ \\
\hline $1^{4} 2^{1} 3^{-4} 6^{5}$ & $\Gamma_{0}(6)$ & $\circ$ & $\circ$ & $\checkmark$ & $\Lambda$ \\
\hline $1^{5} 2^{-4} 3^{1} 6^{4}$ & $\Gamma_{0}(6)$ & $\circ$ & $\circ$ & $\checkmark$ & $\Lambda$ \\
\hline $1^{-2} 2^{4} 3^{-2} 6^{4}$ & $\Gamma_{0}(6)$ & $\circ$ & $\uparrow$ & $\begin{array}{l}\checkmark \\
\checkmark\end{array}$ & $\begin{array}{l}\Lambda_{+} \\
\Lambda_{-}\end{array}$ \\
\hline $1^{-1} 2^{-1} 3^{3} 6^{3}$ & $\Gamma_{0}(6)$ & $\circ$ & $\uparrow$ & $\begin{array}{l}\checkmark \\
\checkmark\end{array}$ & $\begin{array}{l}\Lambda_{+} \\
\Lambda_{-}\end{array}$ \\
\hline $1^{-4} 2^{5} 3^{4} 6^{1}$ & $\Gamma_{0}(6)$ & $0,0^{*}$ & $0,0^{*}$ & $\checkmark$ & $\Lambda$ \\
\hline $2^{3} 6^{3}$ & $\Gamma_{0}(6)_{\mid 2}$ & $\begin{array}{l}\circ \\
\circ\end{array}$ & $\begin{array}{l}\circ \\
\uparrow\end{array}$ & $\begin{array}{l}\checkmark \\
x^{\dagger} \\
L G\end{array}$ & $\begin{array}{l}A_{2}^{12}, A_{3}^{8}, A_{6}^{4}, \Lambda \\
A_{1}^{24}, A_{4}^{6} \\
A_{2}^{12}, D_{4}^{6}, \Lambda_{+}\end{array}$ \\
\hline $6^{4}$ & $\Gamma_{0}(6)_{\mid 6}$ & $\circ$ & $\uparrow$ & $\begin{array}{l}x^{\dagger} \\
x\end{array}$ & $\begin{array}{l}A_{1}^{24}, A_{4}^{6} \\
A_{8}^{3}, \Lambda_{-}\end{array}$ \\
\hline $1^{3} 7^{3}$ & $\Gamma_{0}(7)$ & $\circ$ & $\circ$ & $\checkmark$ & $A_{1}^{24}, A_{3}^{8}, \Lambda$ \\
\hline $1^{2} 2^{1} 4^{1} 8^{2}$ & $\Gamma_{0}(8)$ & $\circ$ & $\circ$ & $\checkmark$ & $A_{1}^{24}, A_{2}^{12}, A_{5}^{4} D_{4}, \Lambda$ \\
\hline $1^{4} 2^{-2} 4^{-2} 8^{4}$ & $\Gamma_{0}(8)$ & $\circ$ & $\uparrow$ & $\begin{array}{r}\times \\
\times\end{array}$ & $\begin{array}{l}\Lambda_{+} \\
\Lambda_{-}\end{array}$ \\
\hline $1^{-2} 2^{3} 4^{1} 8^{2}$ & $\Gamma_{\langle-1\rangle}(8)$ & $\circ$ & $\uparrow$ & $\begin{array}{l}\checkmark \\
\checkmark\end{array}$ & $\begin{array}{l}\Lambda_{+} \\
\Lambda_{-}\end{array}$ \\
\hline $2^{4} 4^{-4} 8^{4}$ & $\Gamma_{0}(8)_{\mid 2}$ & $\begin{array}{l}\circ \\
\circ\end{array}$ & $\begin{array}{l}\circ \\
\circ\end{array}$ & $\begin{array}{l}\checkmark \\
\times\end{array}$ & $\begin{array}{l}\Lambda_{+} \\
\Lambda_{-}\end{array}$ \\
\hline $4^{2} 8^{2}$ & $\Gamma_{0}(8)_{\mid 4}$ & $\circ$ & $\uparrow$ & $\begin{array}{l}x^{\dagger} \\
L G \\
x^{\dagger} \\
x\end{array}$ & $\begin{array}{l}A_{2}^{12} \\
A_{3}^{8}, \Lambda_{+} \\
A_{2}^{12} \\
E_{6}^{4}, \Lambda_{-}\end{array}$ \\
\hline $1^{3} 3^{-2} 9^{3}$ & $\Gamma_{0}(9)$ & $\begin{array}{l}\circ \\
\circ\end{array}$ & $\begin{array}{l}\circ \\
\circ\end{array}$ & $\begin{array}{l}\checkmark \\
\checkmark\end{array}$ & $\begin{array}{l}\Lambda_{+} \\
\Lambda_{-}\end{array}$ \\
\hline $1^{2} 2^{1} 5^{-2} 10^{3}$ & $\Gamma_{\langle-1\rangle}(10)$ & $\circ$ & $\uparrow$ & $\begin{array}{l}\checkmark \\
\checkmark\end{array}$ & $\begin{array}{l}\Lambda_{+} \\
\Lambda_{-}\end{array}$ \\
\hline
\end{tabular}


Table 4 continued

\begin{tabular}{|c|c|c|c|c|c|}
\hline$\pi_{g}$ & $\left(\mathfrak{G}_{g}\right)_{\mid n}$ & $O\left(\Gamma^{4,20}\right)$ classes & $\mathrm{O}^{+}\left(\Gamma^{4,20}\right)$ classes & $\mathcal{Z}_{g}$ & $\phi_{g}^{N}$ \\
\hline $1^{3} 2^{-2} 5^{1} 10^{2}$ & $\Gamma_{\langle-1\rangle}(10)$ & $\circ$ & $\uparrow$ & $\begin{array}{l}\checkmark \\
\checkmark\end{array}$ & $\begin{array}{l}\Lambda_{+} \\
\Lambda_{-}\end{array}$ \\
\hline $1^{-2} 2^{3} 5^{2} 10^{1}$ & $\Gamma_{\langle-1\rangle}(10)$ & $\begin{array}{l}0 \\
{[0]}\end{array}$ & $\begin{array}{l}\uparrow \\
{[\uparrow]}\end{array}$ & $\begin{array}{l}\checkmark \\
\checkmark\end{array}$ & $\begin{array}{l}\Lambda_{+} \\
\Lambda_{-}\end{array}$ \\
\hline $2^{2} 10^{2}$ & $\Gamma_{0}(10)_{\mid 2}$ & {$\left[\begin{array}{l}0 \\
0 \\
0\end{array}\right]$} & {$\left[\begin{array}{l}0 \\
0 \\
0\end{array}\right]$} & $\begin{array}{l}L G \\
\times\end{array}$ & $\begin{array}{l}A_{1}^{24}, A_{2}^{12}, \Lambda_{+} \\
A_{4}^{6} \Lambda_{-}\end{array}$ \\
\hline $1^{2} 11^{2}$ & $\Gamma_{0}(11)$ & {$\left[\begin{array}{l}0 \\
0\end{array}\right]$} & {$\left[\begin{array}{l}0 \\
0\end{array}\right]$} & $\begin{array}{l}\times \\
L G\end{array}$ & $\begin{array}{l}A_{1}^{24}, \Lambda_{+} \\
A_{2}^{12}, \Lambda_{-}\end{array}$ \\
\hline $1^{2} 2^{-2} 3^{2} 4^{2} 6^{-2} 12^{2}$ & $\Gamma_{0}(12)$ & $\circ$ & $\uparrow$ & $\begin{array}{l}\times \\
\times\end{array}$ & $\begin{array}{l}\Lambda_{+} \\
\Lambda_{-}\end{array}$ \\
\hline $1^{1} 2^{2} 3^{1} 4^{-2} 12^{2}$ & $\Gamma_{\langle-1\rangle}(12)$ & $\begin{array}{l}\circ \\
\circ\end{array}$ & $\begin{array}{l}\circ \\
\circ\end{array}$ & $\begin{array}{l}\checkmark \\
\checkmark\end{array}$ & $\begin{array}{l}\Lambda_{+} \\
\Lambda_{-}\end{array}$ \\
\hline $1^{2} 3^{-2} 4^{1} 6^{2} 12^{1}$ & $\Gamma_{\langle-1\rangle}(12)$ & $\begin{array}{l}\circ \\
\circ\end{array}$ & $\begin{array}{l}\circ \\
\circ\end{array}$ & $\begin{array}{l}\checkmark \\
\checkmark\end{array}$ & $\begin{array}{l}\Lambda_{+} \\
\Lambda_{-}\end{array}$ \\
\hline $1^{-2} 2^{2} 3^{2} 4^{1} 12^{1}$ & $\Gamma_{\langle-1\rangle}(12)$ & $\circ$ & $\begin{array}{l}\uparrow \\
\lceil\uparrow\rceil\end{array}$ & $\begin{array}{l}\checkmark \\
\checkmark\end{array}$ & $\begin{array}{l}\Lambda_{+} \\
\Lambda_{-}\end{array}$ \\
\hline $2^{1} 4^{1} 6^{1} 12^{1}$ & $\Gamma_{0}(12)_{\mid 2}$ & {$\left[\begin{array}{l}\circ \\
\circ\end{array}\right]$} & {$\left[\begin{array}{l}\circ \\
\circ\end{array}\right]$} & $\begin{array}{l}\times \\
\times\end{array}$ & $\begin{array}{l}A_{1}^{24}, \Lambda_{+} \\
D_{4}^{6}, \Lambda_{-}\end{array}$ \\
\hline $1^{1} 2^{1} 7^{1} 14^{1}$ & $\Gamma_{0}(14)$ & {$\left[\begin{array}{l}0 \\
0\end{array}\right]$} & {$\left[\begin{array}{l}v \\
0 \\
0\end{array}\right]$} & $\begin{array}{l}\times \\
L G\end{array}$ & $\begin{array}{l}A_{1}^{24}, \Lambda_{+} \\
A_{3}^{8}, \Lambda_{-}\end{array}$ \\
\hline $1^{1} 3^{1} 5^{1} 15^{1}$ & $\Gamma_{0}(15)$ & {$\left[\begin{array}{l}0 \\
0\end{array}\right]$} & $\begin{array}{l}v \\
0 \\
0\end{array}$ & $\begin{array}{l}\times \\
L G\end{array}$ & $\begin{array}{l}A_{1}^{24}, \Lambda_{+} \\
D_{4}^{6}, \Lambda_{-}\end{array}$ \\
\hline
\end{tabular}

An $\times$ denotes a twining function which has not been observed in any K3 NLSM or LG orbifold anywhere in the literature. Finally, for those twining functions which have not been observed in a $K 3 \mathrm{NLSM}$, a ${ }^{\dagger}$ denotes that nevertheless, the explicit twining function is fixed by the modularity arguments of Sect. 4.4.

In the last column, we report the list of Niemeier lattices $N$ such that the given Frame shape appears in the corresponding Niemeier group $G_{N}$. Equivalently, this is the list of those $N$ for which the corresponding Jacobi form $\phi_{g}^{N}$ arising from umbral or Conway moonshine is conjecturally equal to one of the twining genera $\mathcal{Z}_{g}$ of the given Frame shape. When $N=\Lambda$ and the $g$-invariant subspace has dimension exactly 4, we write two different symbols $\Lambda_{+}$and $\Lambda_{-}$to represent the two distinct genera $\phi_{g,+}^{\Lambda} \phi_{g,-}^{\Lambda}$. Niemeier lattices $N$ and $N^{\prime}$ for which $\phi_{g}^{N}=\phi_{g}^{N^{\prime}}$ are listed in the same row. More precisely, next to each $O^{+}\left(\Gamma^{4,20}\right)$-class for which the twining genus $\mathcal{Z}_{g}$ is known, we list all those $N$ for which $\phi_{g}^{N}=\mathcal{Z}_{g}$. In some cases, the same lattice $N$ appears in different rows for the same Frame shape: This occurs whenever two distinct $O^{+}\left(\Gamma^{4,20}\right)$-classes have the same genus 
$\mathcal{Z}_{g}$. Next to the $O^{+}\left(\Gamma^{4,20}\right)$-classes for which the twining genus is unknown, we list those $N$ for which, based on our conjectures, $\phi_{g}^{N}$ is expected to coincide with $\mathcal{Z}_{g}$. For some Frame shapes, $\left(2^{2} 10^{2}, 1^{2} 11^{2}, 2^{1} 4^{1} 6^{1} 12^{1}, 1^{1} 2^{1} 7^{1} 14^{1}, 1^{1} 3^{1} 5^{1} 15^{1}\right)$ we are not able to formulate any reasonable conjecture associating $O^{+}\left(\Gamma^{4,20}\right)$-classes with candidate twining genera $\phi_{g}^{N}$. In these cases, the alignment between classes and lists of Niemeier lattices has no meaning. This is represented by a square parenthesis in the third column. A special case is the Frame shape $1^{-4} 2^{5} 3^{4} 6^{1}$, to which there may correspond either one or two $O\left(\Gamma^{4,20}\right)$ and $\mathrm{O}^{+}\left(\Gamma^{4,20}\right)$ classes; we emphasize our lack of certainty by writing $\circ$, ०*. If there are two, they are not related by world-sheet parity. However, the two classes are inverses of each other, so they have the same twining genus.

Received: 4 April 2017 Accepted: 9 July 2018 Published online: 24 July 2018

\section{References}

1. Ashok, S.K., Troost, J.: A twisted non-compact elliptic genus. JHEP 03, 067 (2011). arXiv:1101.1059 [hep-th]

2. Ashok, S.K., Doroud, N., Troost, J.: Localization and real Jacobi forms. JHEP 04, 119 (2014). arXiv:1311.1110 [hep-th]

3. Aspinwall, P.S.: "K3 surfaces and string duality. In: Fields, Strings and Duality. Proceedings, Summer School, Theoretical Advanced Study Institute in Elementary Particle Physics, TASI'96, Boulder, USA, June 2-28, 1996, pp. $421-540$ (1996). arXiv:hep-th/9611137 [hep-th]

4. Aspinwall, P.S.: Enhanced gauge symmetries and k3 surfaces. Phys. Lett. B 357(3), 329-334 (1995)

5. Aspinwall, P. S.: Enhanced gauge symmetries and K3 surfaces. Phys. Lett. B357, 329-334 (1995). arXiv:hep-th/9507012 [hep-th]

6. Barth, W., Peters, C., Van de Ven, A.: Compact complex surfaces, vol. 4 of Ergebnisse der Mathematik und ihrer Grenzgebiete (3) [Results in Mathematics and Related Areas (3)]. Springer, Berlin (1984). https://doi.org/10.1007/ 978-3-642-96754-2

7. Benjamin, N., Harrison, S.M., Kachru, S., Paquette, N.M., Whalen, D.: On the elliptic genera of manifolds of Spin(7) holonomy. Ann. Henri Poincare 17(10), 2663-2697 (2016). arXiv:1412.2804 [hep-th]

8. Bridgeland, T.: Stability conditions on K3 surfaces. Duke Math. J.141(2), 241-291 (2008) . https://doi.org/10.1215/ S0012-7094-08-14122-5

9. Bruinier, J.H., Funke, J.: On two geometric theta lifts. Duke Math. J. 125(1), 45-90 (2004). https://doi.org/10.1215/ S0012-7094-04-12513-8

10. Chaudhuri, S., Lowe, D .A.: Type IIA heterotic duals with maximal supersymmetry. Nucl. Phys. B459, 113-124 (1996). arXiv:hep-th/9508144 [hep-th]

11. Chaudhuri, S., Polchinski, J.: Moduli space of CHL strings. Phys. Rev. D52, 7168-7173 (1995). arXiv:hep-th/9506048 [hep-th]

12. Chaudhuri, S., Hockney, G., Lykken, J D.: Maximally supersymmetric string theories in $D<10$. Phys. Rev. Lett. 75 2264-2267 (1995). arXiv:hep-th/9505054 [hep-th]

13. Cheng, M.C.N., de Lange, P., Whalen, D.P.Z.: Generalised umbral moonshine. arXiv:1608.07835 [math.RT]

14. Cheng, M.C.N., Dong, X., Duncan, J.F.R., Harrison, S., Kachru, S., Wrase, T.: Mock Modular Mathieu Moonshine Modules. arXiv:1406.5502 [hep-th]

15. Cheng, M.C.N., Duncan, J.F.R., Harrison, S.M., Kachru, S.: Equivariant K3 invariants. arXiv:1508.02047 [hep-th]

16. Cheng, M. C., Duncan, J. F., Harvey, J. A.: Umbral moonshine and the Niemeier lattices. Research in the Mathematical Sciences1(1), 1-81 (2014). arXiv:1307.5793 [math.RT]. https://doi.org/10.1186/2197-9847-1-3

17. Cheng, M.C.N., Ferrari, F., Harrison, S.M., Paquette, N.M.: Landau-Ginzburg Orbifolds and Symmetries of K3 CFTs. arXiv:1512.04942 [hep-th]

18. Cheng, M.C.N., Harrison, S.M., Kachru, S., Whalen, D.: Exceptional Algebra and Sporadic Groups at $\mathrm{c}=12$ arXiv:1503.07219 [hep-th]

19. Cheng, M.C.: K3 Surfaces, N=4 Dyons, and the Mathieu Group M24. Commun. Number Theory Phys. 4, 623-658 (2010). arXiv:1005.5415 [hep-th]

20. Cheng, M.C., Dabholkar, A.: Borcherds-Kac-Moody symmetry of $N=4$ dyons. Commun. Number Theory Phys. 3 59-110 (2009). arXiv:0809.4258 [hep-th]

21. Cheng, M .C., Duncan, J .F.: On Rademacher sums, the largest Mathieu Group, and the Holographic Modularity of Moonshine. Commun. Number Theory Phys. 6, 697-758 (2012). arXiv:1110.3859 [math.RT]

22. Cheng, M.C.N., Harrison, S.: Umbral moonshine and K3 surfaces. Commun. Math. Phys. 339(1), $221-261$ (2015). arXiv:1406.0619 [hep-th]

23. Cheng, M.C., Dong, X., Duncan, J., Harvey, J., Kachru, S., et al.: Mathieu moonshine and N=2 string compactifications. JHEP 1309, 030 (2013). arXiv:1306.4981 [hep-th]

24. Cheng, M .C., Duncan, J .F., Harvey, J .A.: Umbral moonshine. Commun. Number Theory Phys. 08, 101-242 (2014). arXiv:1204.2779 [math.RT]

25. Conway, J.H., Curtis, R.T., Norton, S.P., Parker, R.A., Wilson, R.A.: Atlas of finite groups. Oxford University Press, Eynsham (Maximal subgroups and ordinary characters for simple groups. With computational assistance from J. G, Thackray) (1985) 
26. Conway, J.H., Sloane, N.J.A.: Sphere packings, lattices and groups, vol. 290 of Grundlehren der Mathematischen Wissenschaften [Fundamental Principles of Mathematical Sciences], 3rd edn. Springer-Verlag, New York, third ed. (1999). https://doi.org/10.1007/978-1-4757-6568-7

27. Creutzig, T., Höhn, G.: Mathieu moonshine and the geometry of K3 surfaces. Commun. Number Theory Phys. 08, 295-328 (2014). arXiv:1309.2671 [math.QA]

28. Dabholkar, A., Murthy, S., Zagier, D.: Quantum Black Holes, Wall Crossing, and Mock Modular Forms. arXiv:1208.4074 [hep-th]

29. David, J.R., Sen, A.: CHL dyons and statistical entropy function from D1-D5 system. JHEP 0611, 072 (2006). arXiv:hep-th/0605210 [hep-th]

30. David, J.R., Jatkar, D.P., Sen, A.: Dyon spectrum in $N=4$ supersymmetric Type II string theories. JHEP 11,073 (2006). arXiv:hep-th/0607155 [hep-th]

31. David, J.R., Jatkar, D.P., Sen, A.: Product representation of dyon partition function in CHL models. JHEP 0606, 064 (2006). arXiv:hep-th/0602254 [hep-th]

32. David, J.R., Jatkar, D.P., Sen, A.: Dyon spectrum in generic $N=4$ supersymmetric $Z(N)$ orbifolds. JHEP 01, 016 (2007). arXiv:hep-th/0609109 [hep-th]

33. Dijkgraaf, R., Witten, E.: Topological Gauge theories and group cohomology. Commun. Math. Phys. 129, 393 (1990)

34. Dijkgraaf, R., Vafa, C., Verlinde, E.P., Verlinde, H.L.: The operator algebra of orbifold models. Commun. Math. Phys. 123 485 (1989)

35. Dijkgraaf, R., Verlinde, E.P., Verlinde, H .L.: Counting dyons in N= 4 string theory. Nucl. Phys. B484, 543-561 (1997). arXiv:hep-th/9607026 [hep-th]

36. Dijkgraaf, R., Moore, G .W., Verlinde, E .P., Verlinde, H .L.: Elliptic genera of symmetric products and second quantized strings. Commun. Math. Phys. 185, 197-209 (1997). arXiv:hep-th/9608096 [hep-th]

37. Du Val, P.: Homographies, Quaternions and Rotations. Oxford Mathematical Monographs. Clarendon Press, Oxford (1964)

38. Duncan, J. F. R., Griffin, M. J., Ono, K.: Proof of the Umbral Moonshine Conjecture. arXiv:1503.01472 [math.RT]

39. Duncan, J. F. R., Harvey, J. A.: The Umbral Moonshine Module for the Unique Unimodular Niemeier Root System. arXiv:1412.8191 [math.RT]

40. Duncan, J.F.R., Mack-Crane, S.: The Moonshine Module for Conway's Group. arXiv:1409.3829 [math.RT]

41. Duncan, J.F.: Super-moonshine for Conway's largest sporadic group. Duke Math. J. 139(2), 255-315 (2007)

42. Duncan, J.F., Mack-Crane, S.: Derived equivalences of K3 surfaces and twined elliptic genera. Res. Math. Sci. 3(1), 1-47 (2016)

43. Eguchi, T., Hikami, K.: Note on twisted elliptic genus of K3 surface. Phys. Lett. B 694, 446-455 (2011). arXiv:1008.4924 [hep-th]

44. Eguchi, T., Sugawara, Y.: Non-holomorphic modular forms and SL(2, R)/U(1) superconformal field theory. JHEP 03, 107 (2011). arXiv:1012.5721 [hep-th]

45. Eguchi, T., Ooguri, H., Taormina, A., Yang, S.-K.: Superconformal algebras and string compactification on manifolds with SU(N) holonomy. Nucl. Phys. B 315, 193-221 (1989)

46. Eguchi, T., Ooguri, H., Tachikawa, Y.: Notes on the K3 Surface and the Mathieu group M24. Exp. Math. 20, 91-96 (2011). arXiv:1004.0956 [hep-th]

47. Eichler, M., Zagier, D.: The Theory of Jacobi Forms. Birkhäuser, Basel (1985)

48. Font, A., Ibanez, L.E., Quevedo, F.: String compactifications and N = 2 superconformal coset constructions. Phys. Lett. B 224, 79-88 (1989)

49. Frenkel, I.B., Lepowsky, J., Meurman, A.: A moonshine module for the monster. In: Vertex operators in mathematics and physics, pp. 231-273. Springer (1985)

50. Gaberdiel, M.R., Keller, C.A., Paul, H.: Mathieu Moonshine and Symmetry Surfing. arXiv:1609.09302 [hep-th]

51. Gaberdiel, M.R., Volpato, R.: Mathieu moonshine and orbifold K3s. Contrib. Math. Comput. Sci. 8, 109-141 (2014). arXiv:1206.5143 [hep-th]

52. Gaberdiel, M.R., Hohenegger, S., Volpato, R.: Mathieu twining characters for K3. JHEP 1009, 058 (2010). arXiv:1006.0221 [hep-th]

53. Gaberdiel, M.R., Hohenegger, S., Volpato, R.: Mathieu moonshine in the elliptic genus of K3. JHEP 1010, 062 (2010). arXiv:1008.3778 [hep-th]

54. Gaberdiel, M.R., Hohenegger, S., Volpato, R.: Symmetries of K3 sigma models. Commun. Number Theory Phys. 6, 1-50 (2012). arXiv:1106.4315 [hep-th]

55. Gaberdiel, M.R., Persson, D., Ronellenfitsch, H., Volpato, R.: Generalized Mathieu Moonshine. Commun. Number Theory Phys. 07, 145-223 (2013). arXiv:1211.7074 [hep-th]

56. Gannon, T.: Much ado about Mathieu. arXiv:1211.5531 [math.RT]

57. Gepner, D.: Space-time supersymmetry in compactified string theory and superconformal models. Nucl. Phys. B 296 757 (1988)

58. Ginsparg, P.H.: Applied Conformal Field Theory. In: Les Houches Summer School in Theoretical Physics: Fields, Strings, Critical Phenomena Les Houches, France, June 28-August 5, 1988, pp. 1-168 (1988). arXiv:hep-th/9108028 [hep-th]

59. Hanany, A., Prezas, N., Troost, J.: The partition function of the two-dimensional black hole conformal field theory. JHEP 04, 014 (2002). arXiv:hep-th/0202129 [hep-th]

60. Harrison, S., Kachru, S., Paquette, N.M.: Twining genera of $(0,4)$ supersymmetric sigma models on K3. JHEP $\mathbf{0 4}, 048$ (2014). arXiv:1309.0510 [hep-th]

61. Harvey, J.A., Murthy, S., Nazaroglu, C.: ADE Double Scaled Little String Theories, Mock Modular Forms and Umbral Moonshine. arXiv:1410.6174 [hep-th]

62. Harvey, J.A., Murthy, S.: Moonshine in fivebrane spacetimes. JHEP 1401, 146 (2014). arXiv:1307.7717 [hep-th]

63. Harvey, J.A., Lee, S., Murthy, S.: Elliptic genera of ALE and ALF manifolds from gauged linear sigma models. JHEP 02 110 (2015). arXiv:1406.6342 [hep-th]

64. Hoehn, G., Mason, G.: Finite groups of symplectic automorphisms of hyperkähler manifolds of type $K 3^{[2]}$. arXiv:1409.6055 [math.AG] 
65. Hoehn, G., Mason, G.: The 290 fixed-point sublattices of the Leech lattice. arXiv:1505.06420 [math.GR]

66. Huybrechts, D.: On derived categories of K3 surfaces, symplectic automorphisms and the Conway group. arXiv:1309.6528

67. Jatkar, D.P., Sen, A.: Dyon spectrum in CHL models. JHEP 04, 018 (2006). arXiv:hep-th/0510147 [hep-th]

68. Kachru, S., Paquette, N.M., Volpato, R.: 3D String Theory and Umbral Moonshine. arXiv:1603.07330 [hep-th]

69. Kondō, S., et al.: Niemeier lattices, Mathieu groups, and finite groups of symplectic automorphisms of $K 3$ surfaces. Duke Math. J. 92(3), 593-603 (1998)

70. Miranda, R., Morrison, D.R.: Embeddings of integral quadratic forms (unpublished manuscript). http://web.math.ucsb. edu/ drm/manuscripts/eigf.pdf

71. Miranda, R., Morrison, D.R.: The number of embeddings of integral quadratic forms. II. Proc. Jpn. Acad. Ser. A Math. Sci.62(1), 29-32 (1986). http://projecteuclid.org/euclid.pja/1195514495

72. Miranda, R., Morrison, D.R.: The number of embeddings of integral quadratic forms. I. Proc. Jpn. Acad. Ser. A Math. Sci.61(10), 317-320 (1985). http://projecteuclid.org/euclid.pja/1195514534

73. Mukai, S.: Finite groups of automorphisms of K3 surfaces and the Mathieu group. Inventiones mathematicae $\mathbf{9 4}(1)$, 183-221 (1988)

74. Murthy, S.: A holomorphic anomaly in the elliptic genus. JHEP 06, 165 (2014). arXiv:1311.0918 [hep-th]

75. Nahm, W., Wendland, K.: A Hiker's guide to K3: aspects of $N=(4,4)$ superconformal field theory with central charge $c=6$. Commun. Math. Phys. 216, 85-138 (2001). arXiv:hep-th/9912067 [hep-th]

76. Nikulin, V.V.: Integer symmetric bilinear forms and some of their geometric applications. IzV. Akad. Nauk SSSR Ser. Math.43(1), 111-177 (1979)

77. Nikulin, V.V.: Finite groups of automorphisms of Kählerian K3 surfaces. Trudy Moskov. Mat. Obshch. 38, 75-137 (1979)

78. Nikulin, V.V.: Kählerian K3 surfaces and Niemeier lattices. I. Izvestiya: Math. 77(5), 954 (2013)

79. Ooguri, H., Vafa, C.: Two-dimensional black hole and singularities of CY manifolds. Nucl. Phys. B463, 55-72 (1996). arXiv:hep-th/9511164 [hep-th]

80. Persson, D., Volpato, R.: Fricke S-duality in CHL models. JHEP 12, 156 (2015). arXiv:1504.07260 [hep-th]

81. Roche, P., Pasquier, V., Dijkgraaf, R.: QuasiHopf algebras, group cohomology and orbifold models. Nucl. Phys. Proc. Suppl.18B, 60-72 (1990)

82. Sen, A.: Discrete information from CHL black holes. JHEP 1011, 138 (2010). arXiv:1002.3857 [hep-th]

83. Sen, A., Vafa, C.: Dual pairs of type Il string compactification. Nucl. Phys. B455, 165-187 (1995). arXiv:hep-th/9508064 [hep-th]

84. Shih, D., Strominger, A., Yin, X.: Recounting dyons in N = 4 string theory. JHEP 10, 087 (2006). arXiv:hep-th/0505094 [hep-th]

85. Strominger, A.: Massless black holes and conifolds in string theory. Nucl. Phys. B451, 96-108 (1995). arXiv:hep-th/9504090 [hep-th]

86. Strominger, A., Vafa, C.: Microscopic origin of the Bekenstein-Hawking entropy. Phys. Lett. B379, 99-104 (1996) arXiv:hep-th/9601029 [hep-th]

87. Taormina, A., Wendland, K.: A twist in the M24 moonshine story. arXiv:1303.3221 [hep-th]

88. Taormina, A., Wendland, K.: The overarching finite symmetry group of Kummer surfaces in the Mathieu group $M_{24}$. JHEP 1308, 125 (2013). arXiv:1107.3834 [hep-th]

89. Troost, J.: The non-compact elliptic genus: mock or modular. JHEP 06, 104 (2010). arXiv:1004.3649 [hep-th]

90. Volpato, R:: On symmetries of $\mathcal{N}=(4,4)$ sigma models on $T^{4}$. JHEP 1408, 094 (2014). arXiv:1403.2410 [hep-th]

91. Witten, E.: Some comments on string dynamics. In: Future Perspectives in String Theory. Proceedings, Conference, Strings'95, Los Angeles, USA, March 13-18, 1995, pp. 501-523 (1995). arXiv:hep-th/9507121 [hep-th]

92. Witten, E.: Elliptic genera and quantum field theory. Commun. Math. Phys. 109, 525 (1987)

93. Witten, E.: Phases of $N=2$ theories in two-dimensions. Nucl. Phys. B403, 159-222 (1993). arXiv:hep-th/9301042 [hep-th]

94. Witten, E.: On the Landau-Ginzburg description of $N=2$ minimal models. Int. J. Mod. Phys. A9, 4783-4800 (1994). arXiv:hep-th/9304026 [hep-th]

95. Witten, E.: String theory dynamics in various dimensions. Nucl. Phys. B443, 85-126 (1995). arXiv:hep-th/9503124 [hep-th] 